\title{
Discovery of Chemical Reactions Through Multidimensional Screening
}

\section{Supporting Information}

\author{
Aaron B. Beeler*, Shun Su, Chris A. Singleton, and John A. Porco, Jr.* \\ Department of Chemistry and Center for Chemical Methodology and Library Development \\ (CMLD-BU), Boston University, 590 Commonwealth Avenue, Boston Massachusetts 02215 \\ E-mail:porco@bu.edu
}

General Information: ${ }^{1} \mathrm{H}$ NMR spectra were recorded at $400 \mathrm{MHz}$ at ambient temperature with $\mathrm{CDCl}_{3}$ as solvent unless otherwise stated. ${ }^{13} \mathrm{C}$ NMR spectra were recorded at $100.0 \mathrm{MHz}$ at ambient temperature with $\mathrm{CDCl}_{3}$ as solvent unless otherwise stated. Chemical shifts are reported in parts per million relative to $\mathrm{CDCl}_{3}\left({ }^{1} \mathrm{H}, \delta 7.24 ;{ }^{13} \mathrm{C}\right.$, $\delta$ 77.0). Data for ${ }^{1} \mathrm{H}$ NMR are reported as follows: chemical shift, integration, multiplicity $($ app $=$ apparent, par obsc $=$ partially obscure, ovrlp $=$ overlapping, $\mathrm{s}=$ singlet, $\mathrm{d}=$ doublet, $\mathrm{t}=$ triplet, $\mathrm{q}=$ quartet, $\mathrm{qt}=$ quintuplet, $\mathrm{m}=$ multiplet) and coupling constants are reported as values in hertz. All ${ }^{13} \mathrm{C}$ NMR spectra were recorded with complete proton decoupling. Infrared spectra were recorded on a Nicolet Nexus 670 FT-IR spectrophotometer. High-resolution mass spectra were obtained in the Boston University Chemical Instrumentation Center using a Waters Q-TOF mass spectrometer. Analytical and preparative HPLC were performed on a Waters FractionLynx System with a Waters 600 HPLC pump, MicroMass ZQ 2000 mass spectrometer, Water 996 diode array, and Sedere Sedex 75 ELS detector. A Sunfire 4.6 x $50 \mathrm{~mm} \mathrm{C18}$ column was used for analytical HPLC and a Sunfire 19 x $50 \mathrm{~mm} \mathrm{C18}$ column was used for preparative HPLC. All chromatograms shown were generated using MassLynx 4.1. Melting points were recorded on a Mel-Temp (Laboratory Devices). Analytical thin layer chromatography was performed using $0.25 \mathrm{~mm}$ silica gel $60-\mathrm{F}$ plates. Microwave reactions were carried out using a CEM Explorer/Discover system equipped with a dynamic cooling valve. Flash chromatography of starting materials was performed using an Isco CombiFlash Companion using CombiFlash silica gel cartridges. Otherwise, flash chromatography was performed using 200-400 mesh silica gel (Scientific Absorbent Incorporated). Yields refer to chromatographically and spectroscopically pure materials, unless otherwise stated. Methylene chloride, THF, and toluene were purified by passing through two packed columns of neutral alumina (Innovative Technologies, MA). All non-microwave reactions were carried out in oven-dried glassware under an argon atmosphere unless otherwise noted. Molecular sieve mixtures were prepared under an inert atmosphere using oven-dried $4 \AA$ molecular sieves and metal catalysts $(2.2 \mathrm{mmol}$ metal $/ 1 \mathrm{~g}$ molecular sieves). Supported Liquid extraction (SLE) was carried out using Chem Elute hydromatrix cartridges (Varian Inc.). Analytical reaction matrices were carried out in glass 96-well plates (J-Kem Scientific). Computational structure elucidation was accomplished using ACD Labs Structure Elucidator Version 9 (Advanced Chemistry 
Development). Conformational analysis was carried out using a Molecular Operating Environment (MOE) stochastic search. Chemical names were generated using MDL AutoNom 2000. The Arthur ${ }^{\mathrm{TM}}$ Suite Reaction Planner (Symyx Technologies, Inc.) was used for experimental procedure planning.

\section{Table of Contents}

I. Preparation of $o$-Alkynyl Benzaldehyde Substrates 6 and $28 . \ldots . \ldots \ldots \ldots . . . \ldots . . . .$. S3

II. Analytical Reaction Screening and Profiling...................................S4

III. Products Identified from Known Processes....................................S7

IV. LC-ELSD Profiles for Productive Reactions..................................S8

V. Experimental Procedures and Characterization Data...........................S14

VI. Computational Structure Elucidation.......................................S21

VII. Select NMR Spectra..........................................................S36

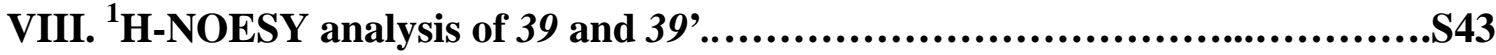

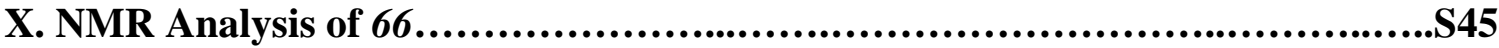

XI. X-ray Crystallographic Data...............................................S48 


\section{Preparation of o-Alkynyl Benzaldehyde Substrates 6 and 28}
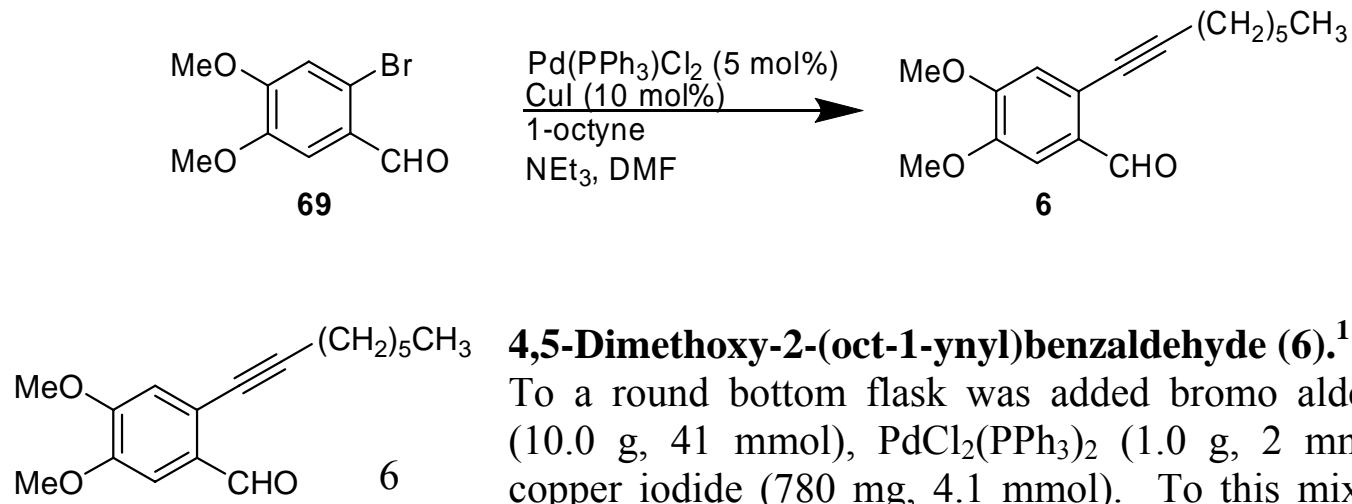

4,5-Dimethoxy-2-(oct-1-ynyl)benzaldehyde (6). ${ }^{1}$

To a round bottom flask was added bromo aldehyde 69 (10.0 g, $41 \mathrm{mmol}), \mathrm{PdCl}_{2}\left(\mathrm{PPh}_{3}\right)_{2}(1.0 \mathrm{~g}, 2 \mathrm{mmol})$, and copper iodide $(780 \mathrm{mg}, 4.1 \mathrm{mmol})$. To this mixture was added DMF $(150 \mathrm{~mL})$ followed by 1-octyne $(7.2 \mathrm{~mL}, 49 \mathrm{mmol})$ and triethylamine (17 $\mathrm{mL}, 120 \mathrm{mmol}$ ). The reaction was stirred at $60{ }^{\circ} \mathrm{C}$ for $24 \mathrm{~h}$, quenched with $1 \mathrm{~N} \mathrm{HCl}$, and stirred for $30 \mathrm{~min}$. The solution was diluted with EtOAc and washed with water (x5) and brine (x2). The organic layer was dried over sodium sulfate, filtered and evaporated in vacuo. The crude material was purified by flash chromatography $\left(\mathrm{SiO}_{2}, 0-20 \%\right.$ EtOAc/petroleum ether) to afford o-alkynyl benzaldehyde $6(81 \%, 9.1 \mathrm{~g}, 33 \mathrm{mmol})$ as a yellowish solid. m.p. $=43-46{ }^{\circ} \mathrm{C}$; ${ }^{1} \mathrm{H}$ NMR $\left(400 \mathrm{MHz}, \mathrm{CDCl}_{3}\right) \delta 10.37(\mathrm{~s}, 1 \mathrm{H}), 10.14$ $(\mathrm{s}, 1 \mathrm{H}), 7.28$ (dd, $1 \mathrm{H}, J=1.44,20.70 \mathrm{~Hz}), 7.12(\mathrm{~s}, 1 \mathrm{H}), 6.92(\mathrm{~s}, 1 \mathrm{H}), 4.00(\mathrm{~s}, 3 \mathrm{H})$, $3.75(\mathrm{~s}, 3 \mathrm{H}), 2.46(\mathrm{t}, 2 \mathrm{H}, J=7.12 \mathrm{~Hz}), 2.04(\mathrm{~m}, 2 \mathrm{H}), 1.43(\mathrm{~m}, 6 \mathrm{H}), 0.90(\mathrm{t}, 3 \mathrm{H}, J=$ $6.99 \mathrm{~Hz}) ;{ }^{13} \mathrm{C}$ NMR $\left(100 \mathrm{MHz}, \mathrm{CDCl}_{3}\right) \delta 191.1,153.8,149.4,130.4,122.9,114.6,108.2$, 96.8, 76.3, 56.4, 56.3, 31.5, 28.9, 28.8, 22.7, 19.8, 14.3; HRMS calculated for $\mathrm{C}_{21} \mathrm{H}_{25} \mathrm{NO}_{5}$ : 274.1569, found: $275.1647(\mathrm{M}+\mathrm{H})$.

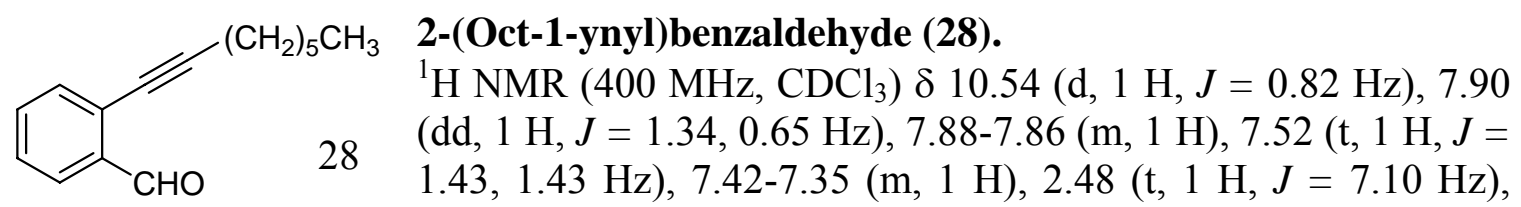

$1.64(\mathrm{qd}, 2 \mathrm{H}, J=14.50,7.25 \mathrm{~Hz}$ ), 1.52-1.38 (m, $2 \mathrm{H}), 1.38-1.26(\mathrm{~m}, 6 \mathrm{H}), 0.91(\mathrm{t}, 3 \mathrm{H} J$

$=6.00 \mathrm{~Hz}) ;{ }^{13} \mathrm{C} \mathrm{NMR}\left(100 \mathrm{MHz}, \mathrm{CDCl}_{3}\right) \delta 192.4,136.2,133.9,133.5,128.2,128.1$, $127.1,98.5,76.5,31.5,28.9,28.7,22.8,19.8,14.3$; HRMS calculated for $\mathrm{C}_{15} \mathrm{H}_{18} \mathrm{O}$ : 214.1358, found: $215.1436(\mathrm{M}+\mathrm{H})$.

(1) For examples of Sonogashira coupling of o-bromobenzaldehydes and alkynes, see: (a) Fkyerat, A.; Dubin, G.; Tabacchi, R. Helv. Chem. Acta 1999, 82, 1418. (b) Zhu, J.; Grigoriadis, N. P.; Lee, J. P.; Porco, J. A., Jr. J. Am. Chem. Soc. 2005, 127, 9342. 


\section{Analytical Reaction Screening and Profiling}

General procedure for analytical reaction screening: Glass 96-well plates were prepared by addition of $10 \mu \mathrm{L}$ of a $0.5 \mathrm{M}$ solution $\left(\mathrm{C}_{2} \mathrm{H}_{4} \mathrm{Cl}_{2}\right)$ of $o$-alkynyl benzaldehyde 5 ( $\left.5 \mu \mathrm{mol}\right)$ in dry dichloroethane in a nitrogen purged glove box. To the 96-well plates was added $10 \mu \mathrm{L}$ of a $0.7 \mathrm{M}$ solution $\left(\mathrm{C}_{2} \mathrm{H}_{4} \mathrm{Cl}_{2}\right)$ of each reaction partner and $5 \mathrm{mg}$ of catalyst $/ 4 \AA$ molecular sieve mixtures $(2.2 \mathrm{mmol} / \mathrm{g}, 1 \mu \mathrm{mol})$. 96-well plates were sealed with individual septa and mixed on an orbital shaker for $3 \mathrm{~h}$. Reaction mixtures were applied to preconditioned (saturated sodium bicarbonate) hydromatrix cartridges and the products eluted with $1.5 \mathrm{~mL}$ of $\mathrm{CH}_{2} \mathrm{Cl}_{2}$. The solutions were evaporated, dissolved in $1 \mathrm{~mL}$ of acetonitrile, and analyzed by LC/MS/ELSD $\left(60->85 \% \mathrm{CH}_{3} \mathrm{CN}, 10 \mathrm{~min}\right)$.

\section{a) LC-ELSD Profile for Terminal Alkyne 15}
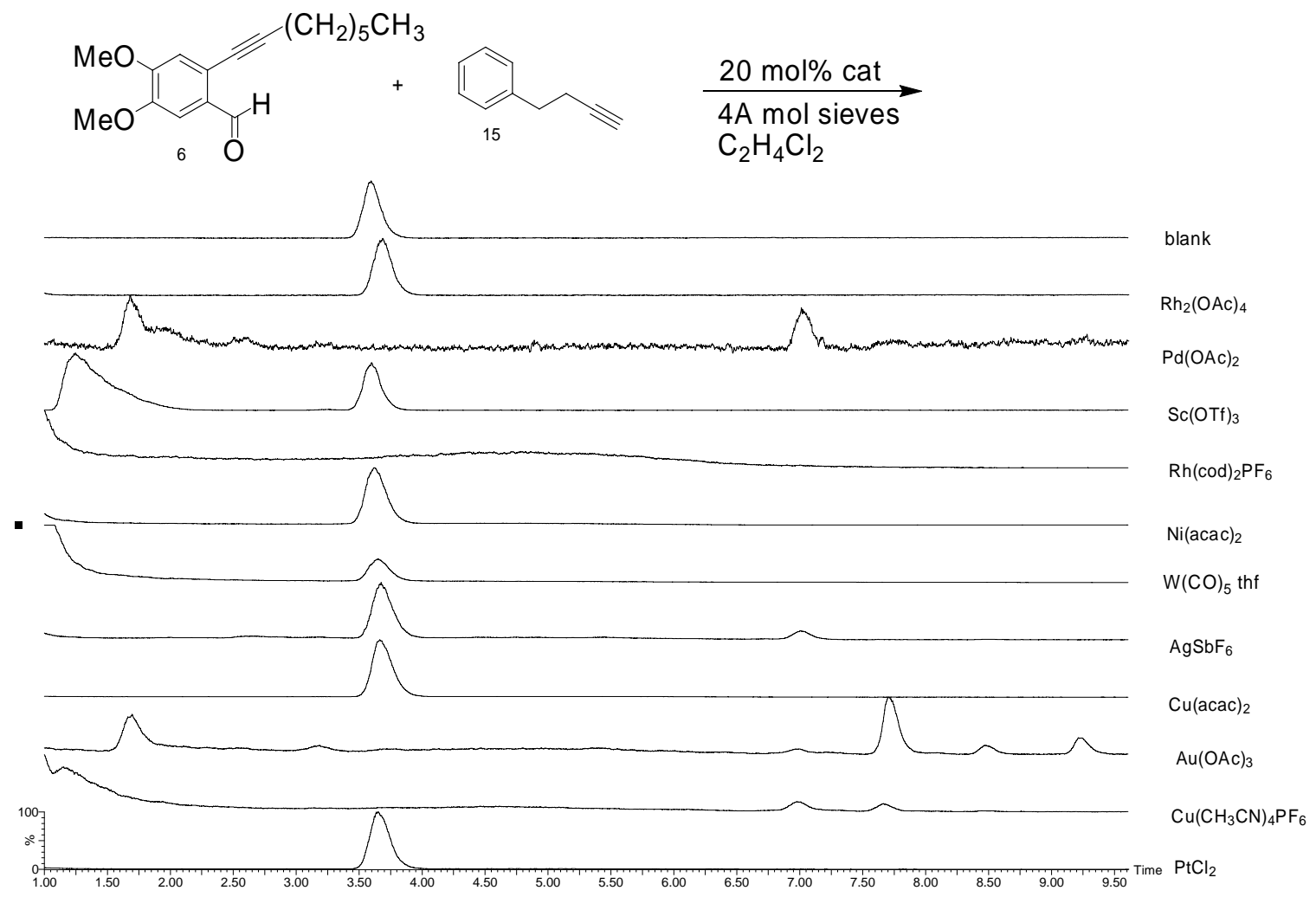
b) LC-ELSD Profile for Diethyl Malonate 13

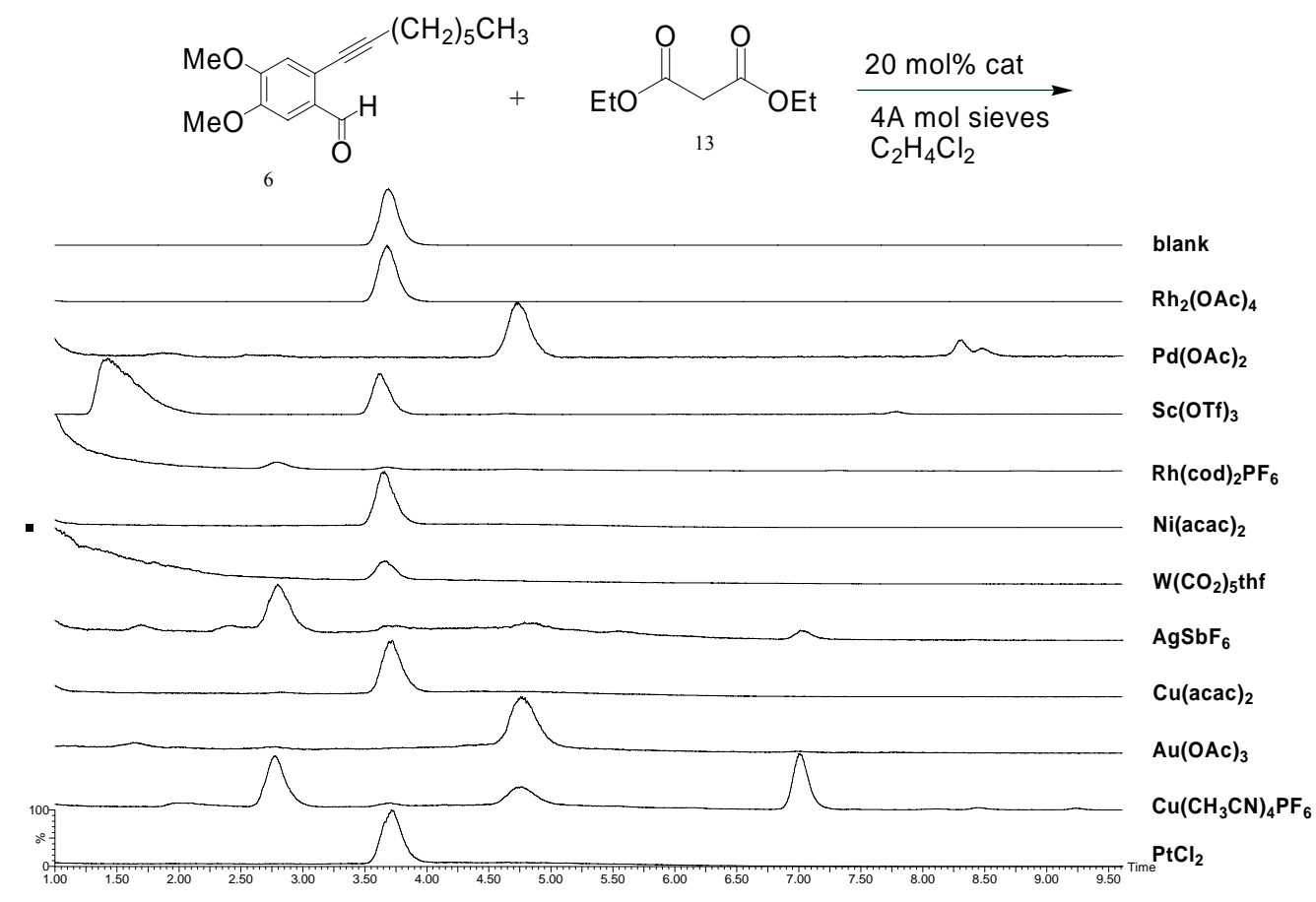

\section{c) LC-ELSD Profile for Methyl Indole 12}

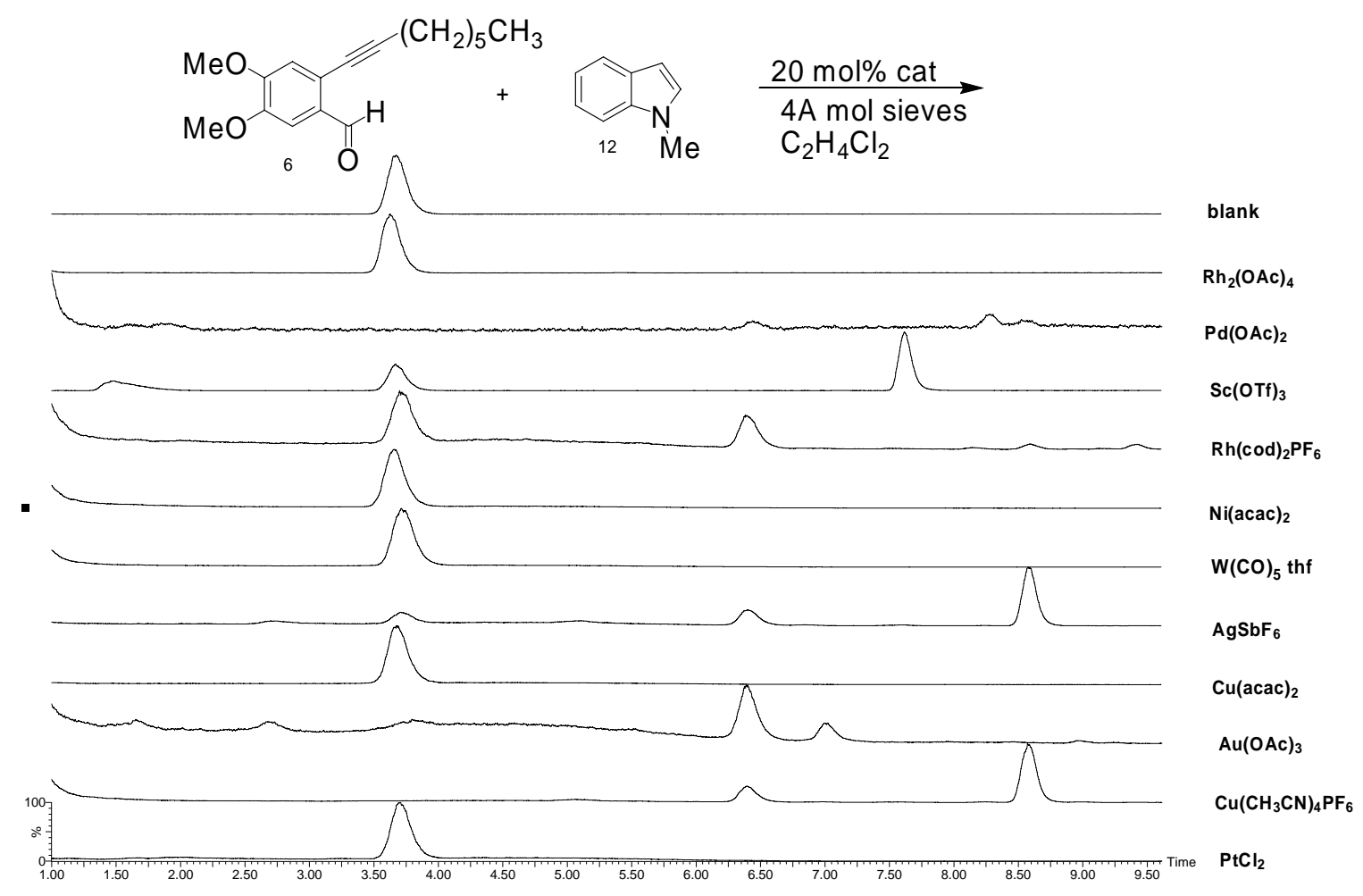


d) Full Reaction Screening Profile

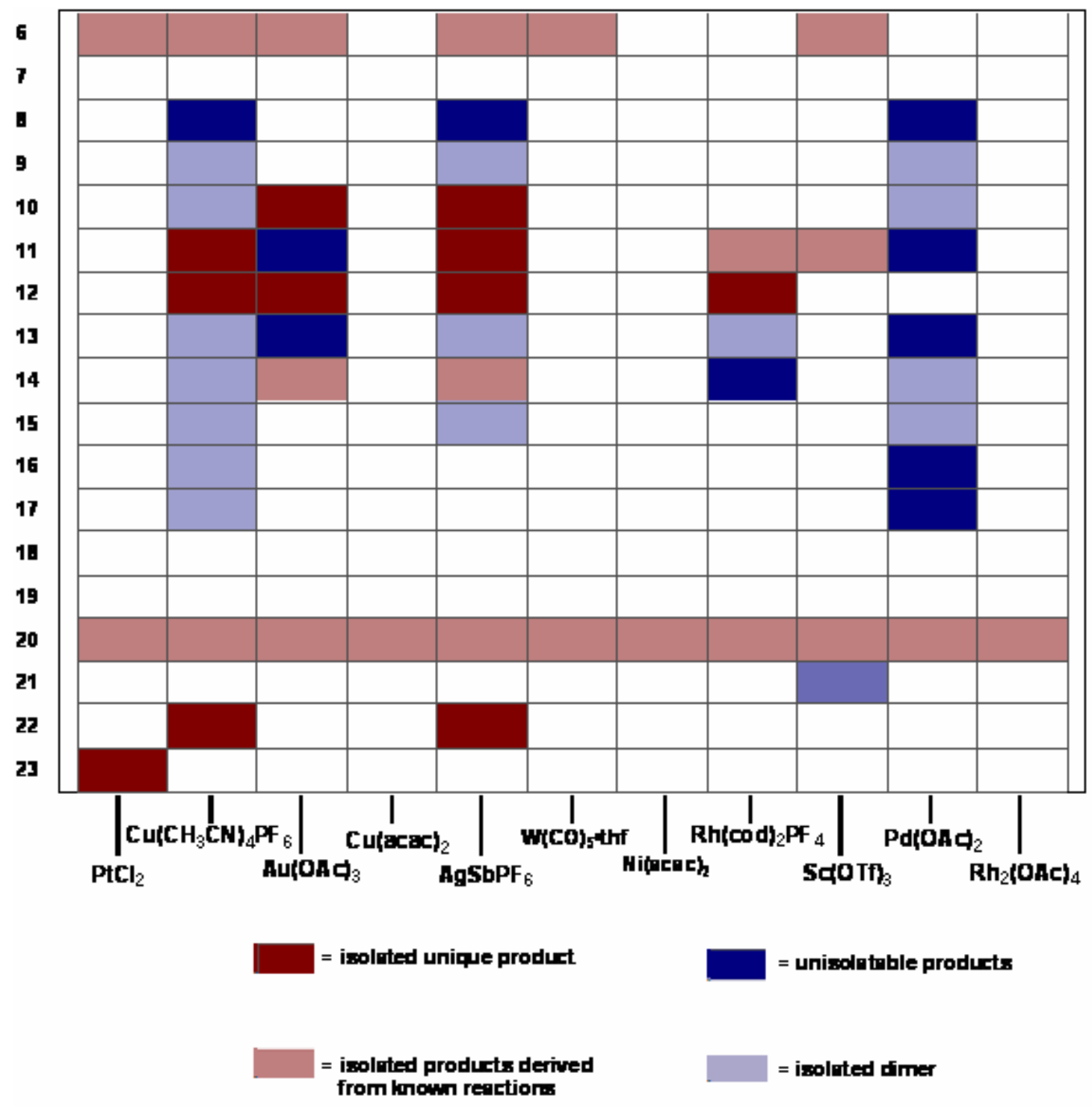




\section{Products Identified from Known Processes ${ }^{2}$}

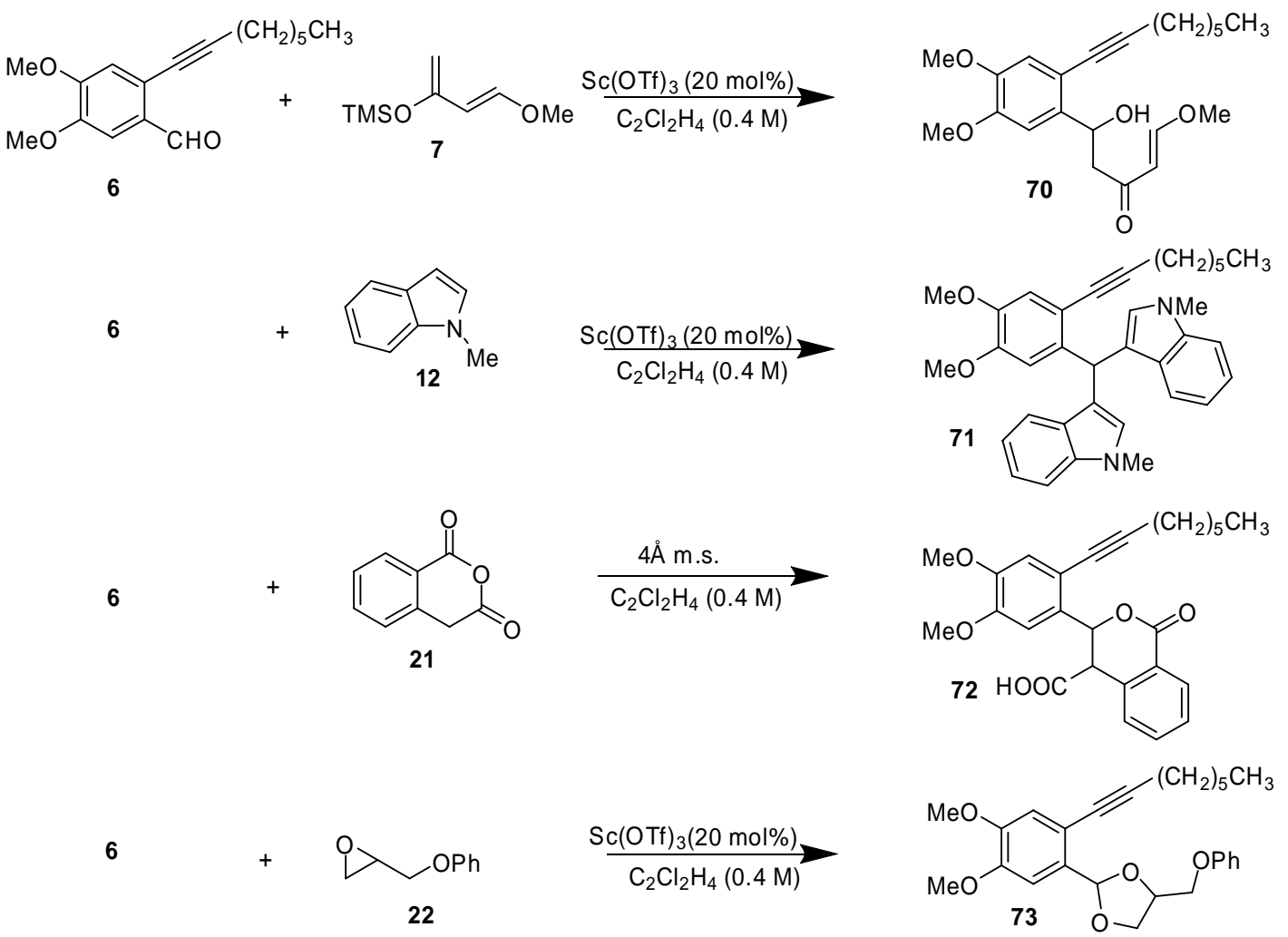

(2) (a) Yang, W.; Shang, D.; Liu, Y.; Du, Y.; Feng, X. J. Org. Chem. 2005, 70, 8533. (b) Lie, W.; Lin, X.F.; Wang, J.; Li, G.-L.; Wang, Y.-G. Syn. Comm. 2005, 35, 2765. (c) Azizian, J.; Mohammadi, A. A.;

Karimi, A. R.; Mohammadizadeh, M. R. J. Org. Chem. 2005, 70, 350. (d) Mucsi, I.; Meleg, A.; Molnar, A.; Bartok, M. J. Mol. Cat. A 2001, 168, 47. 
IV. LC-ELSD Profiles for Productive Reactions

a) Product Profile for Enol Ether 11
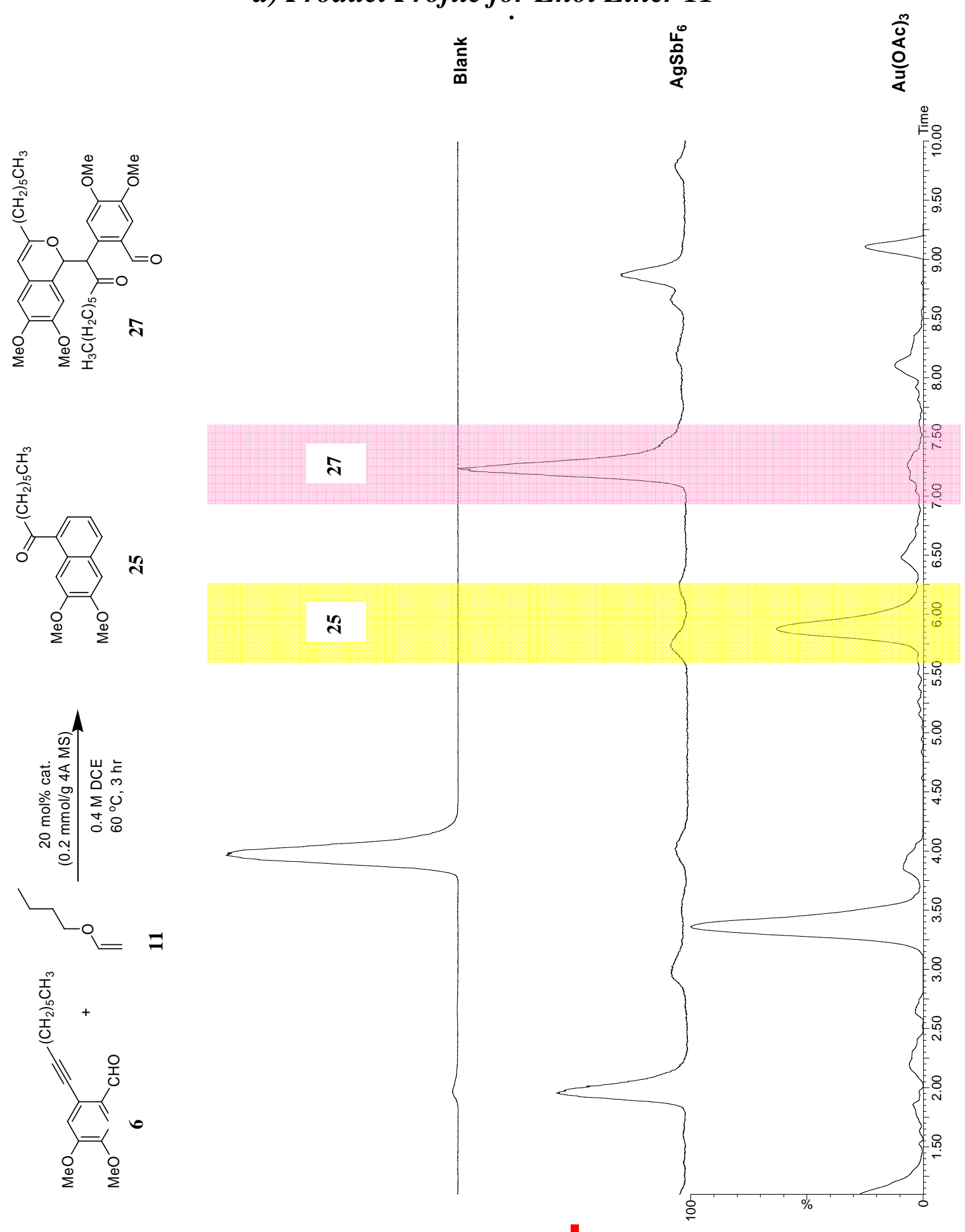


\section{b)Product Profile for Alkyne 16}
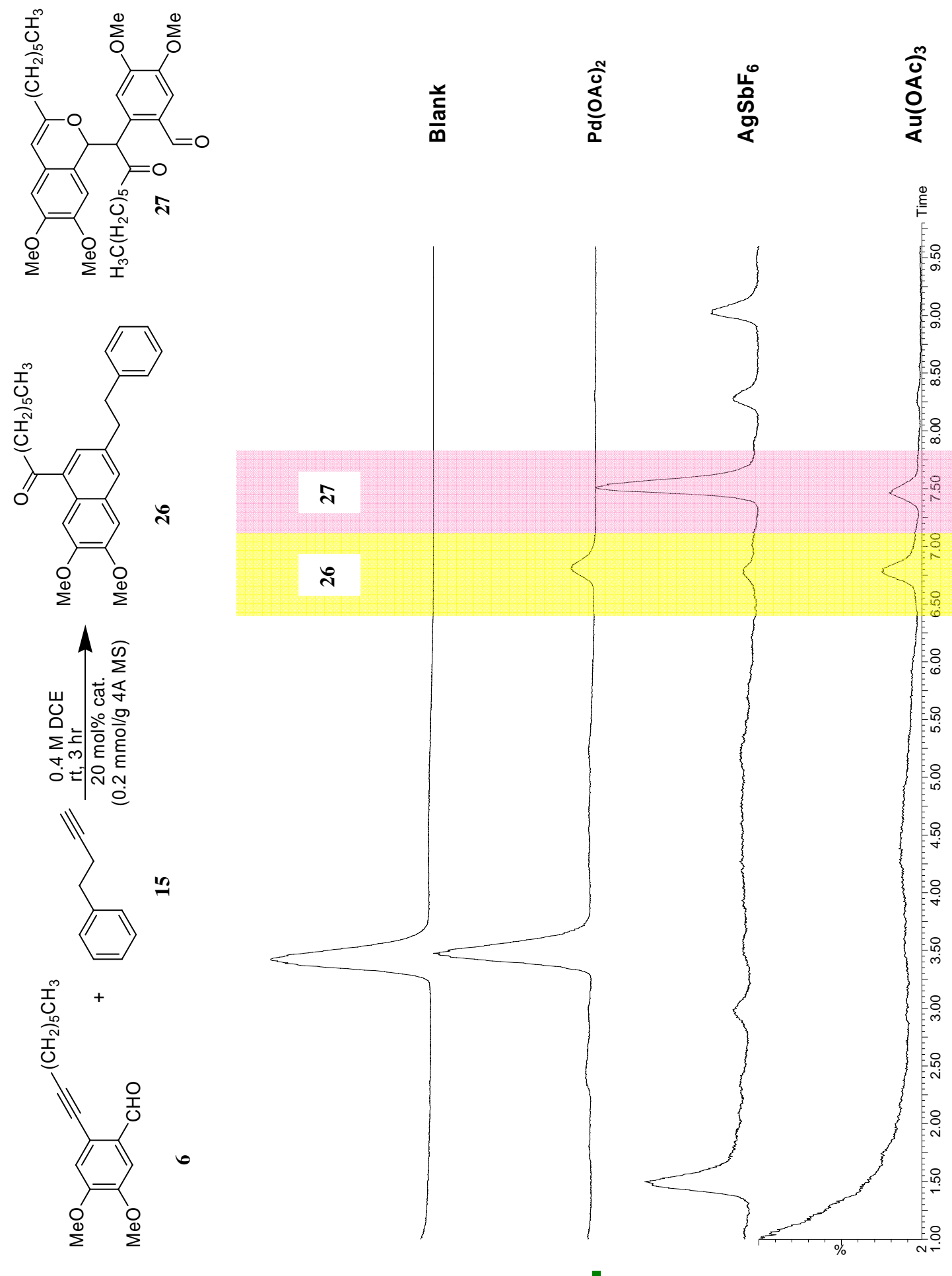


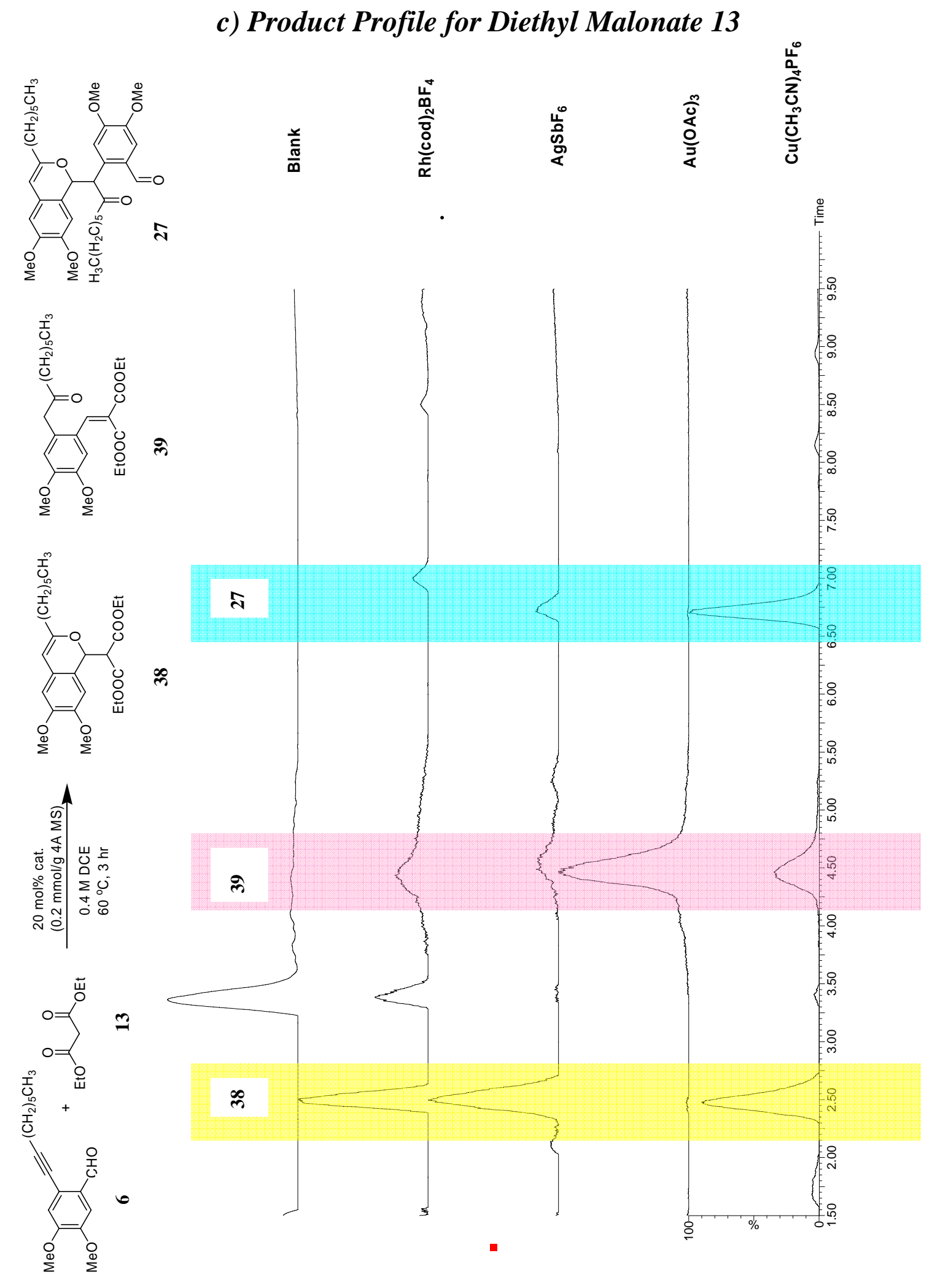




\section{d) Product Profile for Methyl Indole 6}

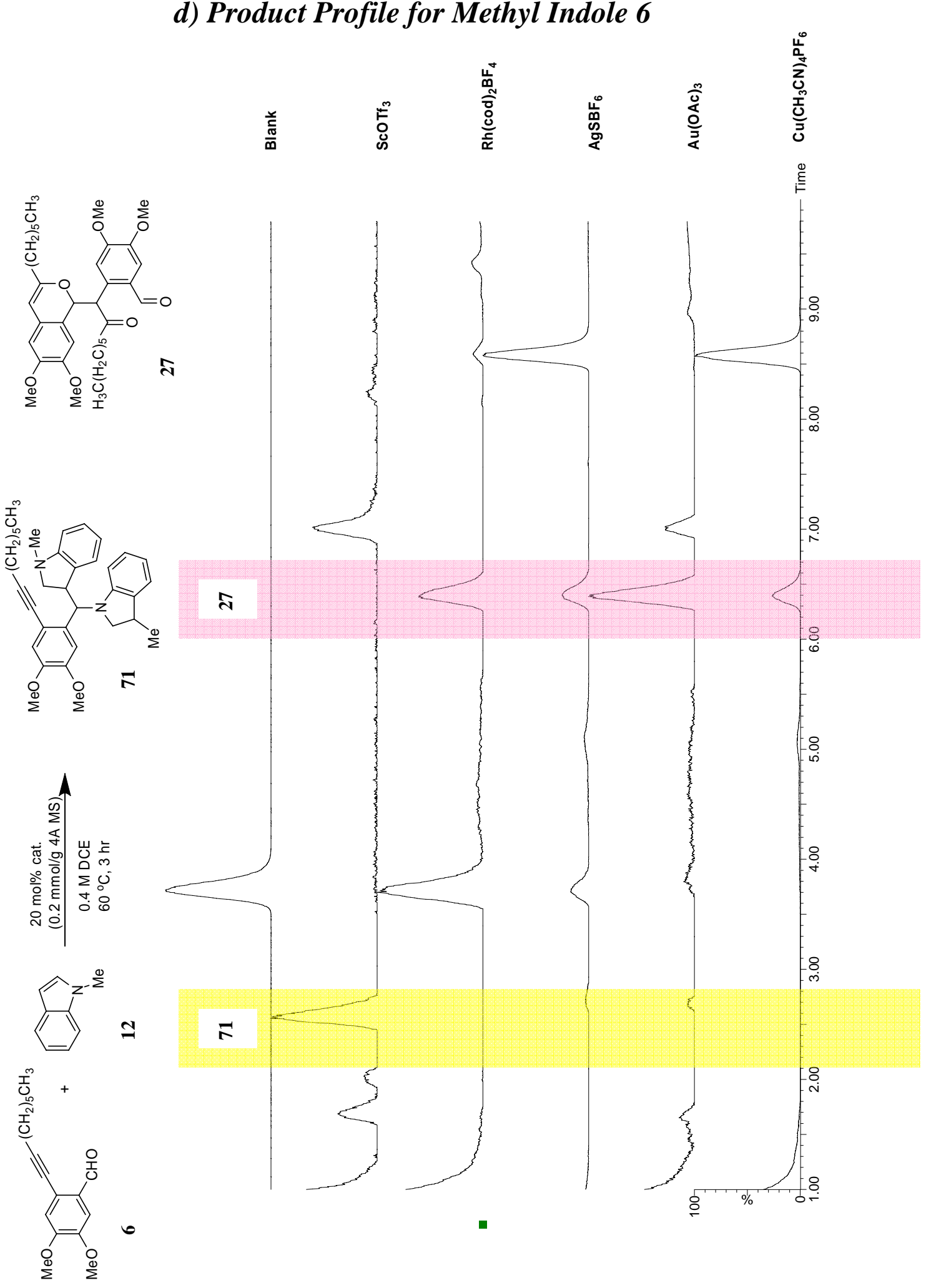


e) Product Profile for Dimethyl Aniline 24

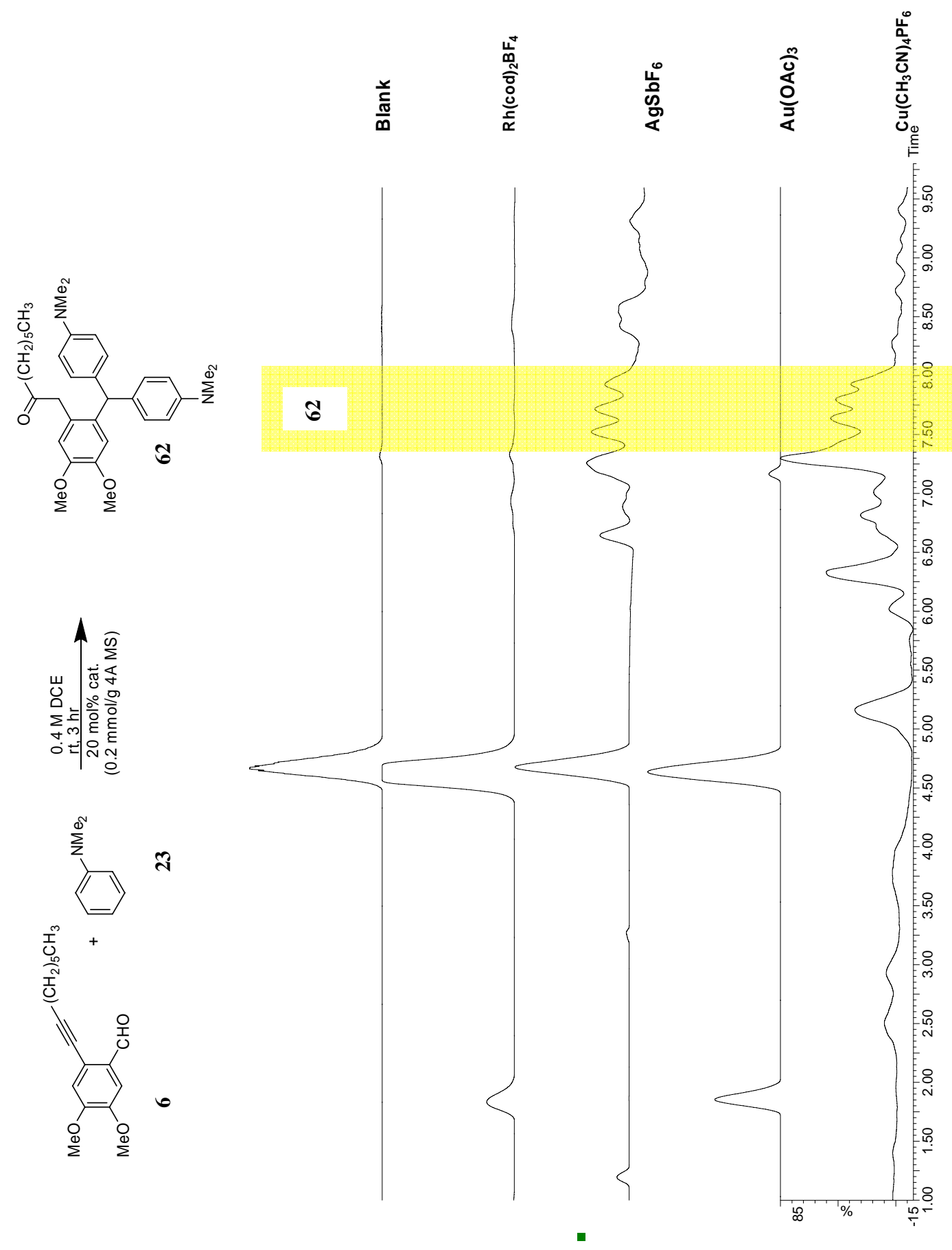




\section{f) Product Profile for Phenol 25}

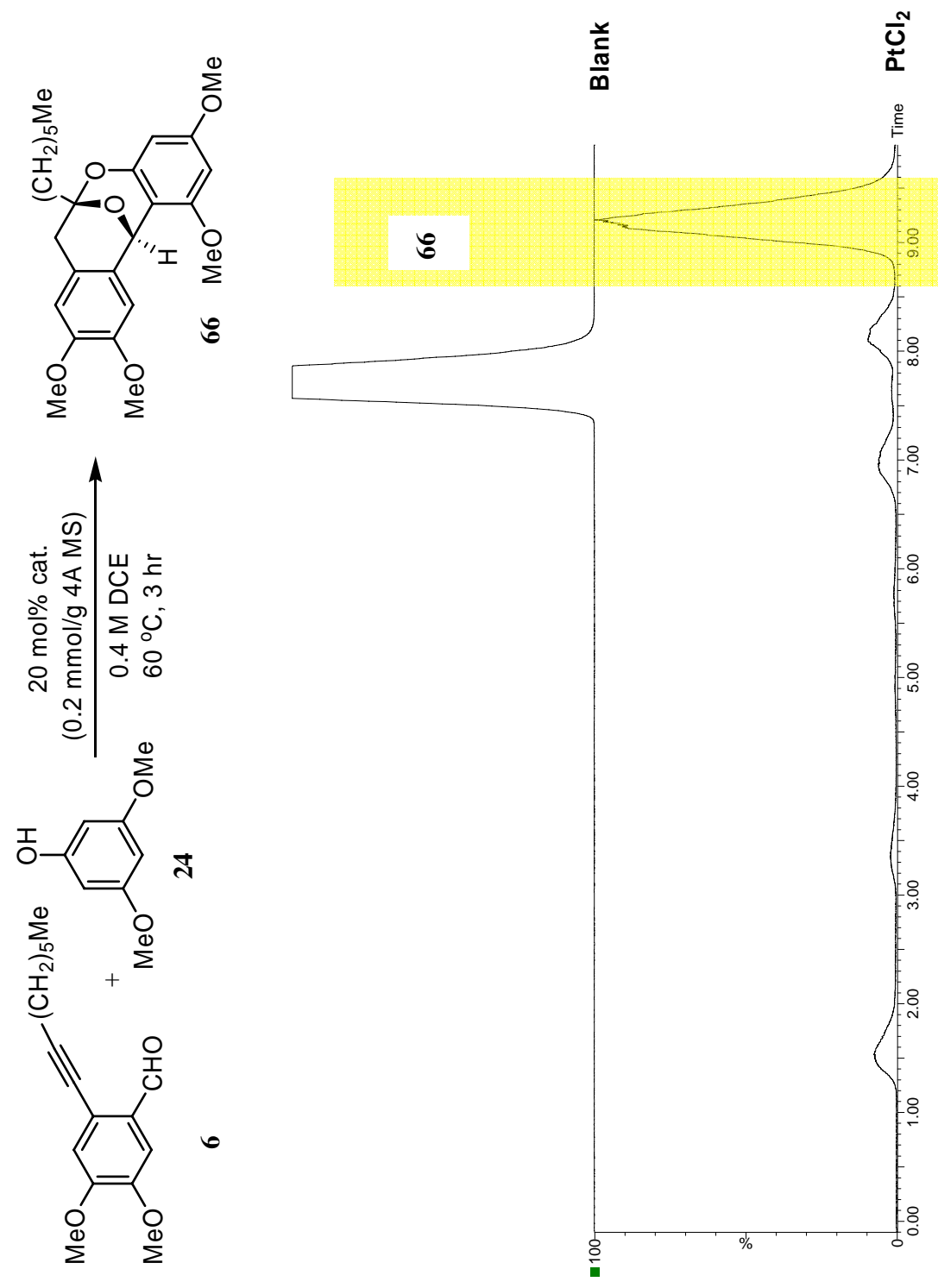




\section{Experimental Procedures and Characterization Data}

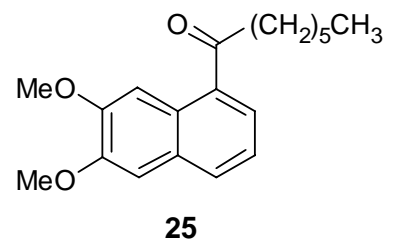

1-(2,3-Dimethoxynaphthalen-5-yl)heptan-1-one (25).

To a flame-dried reaction vessel was added alkynyl benzaldehyde 6 ( $20.0 \mathrm{mg}, 0.073 \mathrm{mmol})$, vinyl ether $11(14 \mu \mathrm{L}$, $0.11 \mathrm{mmol})$, gold (III) chloride $(2.2 \mathrm{mg}, 0.0073 \mathrm{mmol})$, and dichloroethane $(400 \mu \mathrm{L})$. The reaction was stirred at $\mathrm{rt}$ for $4 \mathrm{~h}$ and the solvent evaporated in vacuo. The crude material was purified by flash chromatography $\left(\mathrm{SiO}_{2}, 25: 75 \mathrm{EtOAc} /\right.$ petroleum ether) to afford naphthyl ketone 25 (64 $\%, 14 \mathrm{mg}, 0.047 \mathrm{mmol})$ as a yellow oil. ${ }^{1} \mathrm{H} \mathrm{NMR}\left(400 \mathrm{MHz}, \mathrm{CDCl}_{3}\right) \delta 8.28(\mathrm{~s}, 1 \mathrm{H}), 7.85$ (dd, $1 \mathrm{H}, J=7.77,2.88 \mathrm{~Hz}$ ), 7.36 (dd, $1 \mathrm{H}, J=8.04,7.45 \mathrm{~Hz}), 7.14$ (s, $1 \mathrm{H}), 4.03$ (s, 3 H), 4.01 (s, $3 \mathrm{H}), 3.07$ (m, $2 \mathrm{H}), 1.79$ (m, $2 \mathrm{H}), 1.36$ (ddd, $6 \mathrm{H}, J=10.96,10.10,4.99$ $\mathrm{Hz}), 0.89$ (t, $3 \mathrm{H}, J=7.07 \mathrm{~Hz})$; $\left(100.0 \mathrm{MHz}, \mathrm{CDCl}_{3}\right) 205.2,151.3,149.7,133.5,131.5$, 130.6, 127.1, 126.7, 123.0, 106.8, 105.1, 56.2, 56.0, 41.9, 31.9, 29.9, 29.3, 25.1, 22.8, 14.3; IR (thin film) vmax $\mathrm{cm}^{-1}$; HRMS calculated for $\mathrm{C}_{19} \mathrm{H}_{24} \mathrm{O}_{3}: 300.1725$, found: $301.1894(\mathrm{M}+\mathrm{H})$.

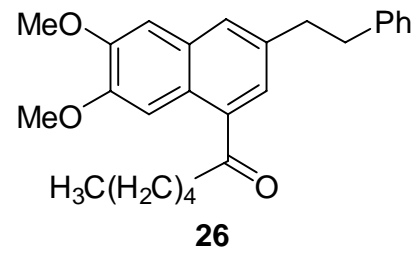

\section{1-(2,3-dimethoxy-6-phenethylnaphthalen-8-yl)hexan-1-one} (26).

$1 \mathrm{H}{ }^{1} \mathrm{H}$ NMR $\left(400 \mathrm{MHz}, \mathrm{CDCl}_{3}\right) \delta 8.19(\mathrm{~s}, 1 \mathrm{H}), 7.65$ (t, $J=3.55,3.55 \mathrm{~Hz}, 1 \mathrm{H}), 7.30$ (s, $1 \mathrm{H}), 7.21(\mathrm{~s}, 1 \mathrm{H}), 7.08$ (s, 1 $\mathrm{H}), 5.30(\mathrm{~s}, 1 \mathrm{H}), 4.01(\mathrm{~s}, 1 \mathrm{H}), 4.00(\mathrm{~s}, 1 \mathrm{H}), 3.05(\mathrm{~s}, 1 \mathrm{H}), 2.81$ (s, $1 \mathrm{H}), 2.70(\mathrm{~s}, 1 \mathrm{H}), 2.05$ (s, $1 \mathrm{H}), 1.78$ (td, $J=14.99,7.47$, $7.47 \mathrm{~Hz}, 1 \mathrm{H}), 1.41$ (s, $1 \mathrm{H}), 1.33$ (s, $1 \mathrm{H}), 0.89$ (t, J=7.08, $7.08 \mathrm{~Hz}, 1 \mathrm{H}) ;(100.0 \mathrm{MHz}$, $\left.\mathrm{CDCl}_{3}\right) \delta 130.3,128.7,128.3,126.1,125.1,106.4,105.0,56.1,56.0,41.9,35.6,35.4$, 33.1, 31.9, 29.3, 25.1, 22.8, 14.3; IR (thin film) vmax $\mathrm{cm}^{-1}$; HRMS calculated for $\mathrm{C}_{26} \mathrm{H}_{30} \mathrm{O}_{3}: 390.2195$, found: $(\mathrm{M}+\mathrm{H})$.

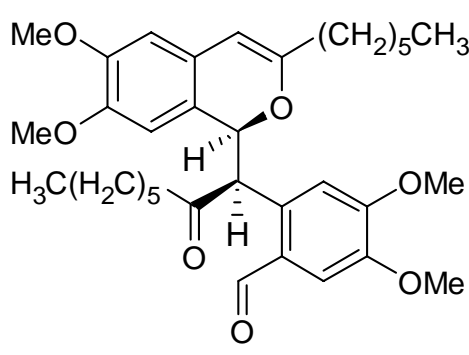

27
2-(1-(3-Hexyl-6,7-dimethoxy-1H-isochromen-1-yl)-2oxooctyl)-4,5-dimethoxybenzaldehyde (27).

To a reaction vessel was added alkynyl benzaldehyde $\mathbf{6}$ (40.0 mg, $0.15 \mathrm{mmol}$ ), AgOTf (14 mg, $0.06 \mathrm{mmol}), \mathrm{H}_{2} \mathrm{O}$ $(2.7 \mu \mathrm{L}, 0.15 \mathrm{mmol})$, and $\mathrm{CH}_{3} \mathrm{CN}(500 \mu \mathrm{L})$. The reaction was stirred for $3 \mathrm{~h}$ at $\mathrm{rt}$ and the solvent evaporated in vacuo. The crude material was purified by flash chromatography $\left(\mathrm{SiO}_{2}, 40: 60 \mathrm{EtOAc} /\right.$ petroleum ether) to afford dimer $27(71 \%, 29 \mathrm{mg}, 0.11 \mathrm{mmol})$ as a yellow

oil. ${ }^{1} \mathrm{H}$ NMR $\left(400 \mathrm{MHz}, \mathrm{CDCl}_{3}\right) \delta 9.61(\mathrm{~s}, 1 \mathrm{H}), 7.12(\mathrm{~s}, 1 \mathrm{H}), 6.97(\mathrm{~s}, 1 \mathrm{H}), 6.40(\mathrm{~s}, 1 \mathrm{H})$, $5.70(\mathrm{dd}, 2 \mathrm{H}, J=48.87,9.59 \mathrm{~Hz}), 5.59(\mathrm{~s}, 1 \mathrm{H}), 5.48(\mathrm{~s}, 1 \mathrm{H}), 3.97(\mathrm{~s}, 3 \mathrm{H}), 3.87$ (s, 3 H), $3.79(\mathrm{~s}, 3 \mathrm{H}), 3.33(\mathrm{~s}, 3 \mathrm{H}), 2.45(\mathrm{dt}, 2 \mathrm{H}, J=7.12,2.83 \mathrm{~Hz}), 2.16(\mathrm{~m}, 2 \mathrm{H}), 1.54(\mathrm{~m}$, $2 \mathrm{H}), 1.32(\mathrm{~m}, 8 \mathrm{H}), 1.21(\mathrm{~m}, 8 \mathrm{H}), 0.89$ (t, $3 \mathrm{H}, J=6.82 \mathrm{~Hz}), 0.83$ (t, $3 \mathrm{H}, J=6.93 \mathrm{~Hz}$ );

${ }^{13} \mathrm{C}$ NMR $\left(100.0 \mathrm{MHz}, \mathrm{CDCl}_{3}\right) \delta 208.6,188.7,154.0,153.5,149.2,148.7,146.5,131.0$, $128.9,124.1,118.1,112.2,109.3,106.4,99.6,78.8,56.7,56.2,56.0,56.0,44.4,34.1$, $31.9,31.7,29.3,29.0,27.4,23.6,22.8,22.7,14.3,14.2$; IR (thin film) vmax $\mathrm{cm}^{-1}$; HRMS calculated for $\mathrm{C}_{34} \mathrm{H}_{46} \mathrm{O}_{7} 566.3244$, found: $589.3077(\mathrm{M}+\mathrm{Na})$. 


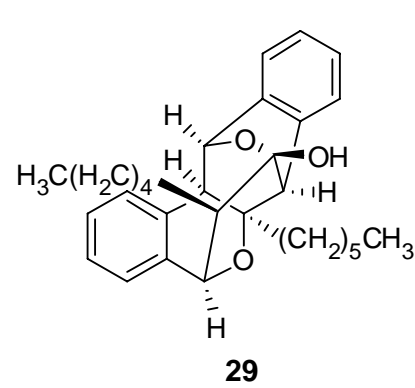

\section{Homodimer (39)}

To a reaction vessel was added alkynyl benzaldehyde 28 (40.0 $\mathrm{mg}, 0.19 \mathrm{mmol})$ and $\operatorname{AgOTf}(14 \mathrm{mg}, 0.04 \mathrm{mmol}), \mathrm{H}_{2} \mathrm{O}(3 \mu \mathrm{L}$, $0.19 \mathrm{mmol})$ and $\mathrm{CH}_{3} \mathrm{CN}(500 \mu \mathrm{L})$. The reaction was stirred for $5 \mathrm{~h}$ at $\mathrm{rt}$ and the solvent evaporated in vacuo. The crude material was flash chromatographed $\left(\mathrm{SiO}_{2}, \quad 40: 60\right.$ EtOAc/petroleum ether) to afford dimer $29(50 \%, 21 \mathrm{mg}$, $0.095 \mathrm{mmol})$ as a yellow oil. ${ }^{1} \mathrm{H} \mathrm{NMR}\left(400 \mathrm{MHz}, \mathrm{CDCl}_{3}\right) \delta$ $7.36(\mathrm{~m}, 6 \mathrm{H}), 7.21(\mathrm{~m}, 2 \mathrm{H}), 4.87(\mathrm{~s}, 1 \mathrm{H}), 4.49(\mathrm{~d}, 1 \mathrm{H}, J=$ $1.70 \mathrm{~Hz}), 3.32(\mathrm{~s}, 1 \mathrm{H}), 2.75(\mathrm{~d}, 1 \mathrm{H}, J=1.61 \mathrm{~Hz}), 2.07(\mathrm{~s}, 1 \mathrm{H}), 1.84(\mathrm{~s}, 1 \mathrm{H}), 1.65(\mathrm{~s}, 1$ $\mathrm{H}), 1.39(\mathrm{~s}, 1 \mathrm{H}), 1.18(\mathrm{~s}, 1 \mathrm{H}), 0.94(\mathrm{t}, 3 \mathrm{H}, J=6.94 \mathrm{~Hz}), 0.80(\mathrm{t}, 3 \mathrm{H}, J=6.94 \mathrm{~Hz}) ;{ }^{13} \mathrm{C}$ NMR $\left(100.0 \mathrm{MHz}, \mathrm{CDCl}_{3}\right) \delta 139.6,139.4,135.8,134.5,128.7,128.3,128.2,128.0$, $127.8,126.5,125.8,123.4,96.2,78.0,75.4,75.0,32.4,31.9,31.8,29.7,29.4,28.0,24.3$, 22.8, 22.7, 22.4, 14.4, 14.2; IR (thin film) vmax 2926, 2859, 1698, 1650, 1459, 1103, 1010, $747 \mathrm{~cm}^{-1}$; HRMS calculated for $\mathrm{C}_{30} \mathrm{H}_{38} \mathrm{O}_{3}$ : 446.2821, found: $469.2719(\mathrm{M}+\mathrm{Na})$. See H-NOESY data (page S43) for complete stereochemical assignment.

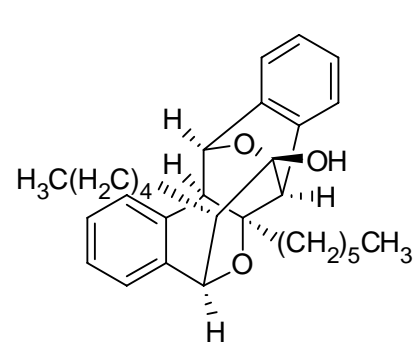

29'

\section{Homodimer (29)}

m.p. $={ }^{1} \mathrm{H}$ NMR $\left(400 \mathrm{MHz}, \mathrm{CDCl}_{3}\right) \delta 7.26$ (s, $\left.1 \mathrm{H}\right), 7.13$ (ddd, $1 \mathrm{H}, J=16.54,12.28,4.67 \mathrm{~Hz}), 7.26(\mathrm{~m}, 1 \mathrm{H}), 4.91(\mathrm{~d}, J=$ $6.07 \mathrm{~Hz}, 1 \mathrm{H}), 4.44(\mathrm{~d}, 1 \mathrm{H}, J=1.81 \mathrm{~Hz}), 3.13(\mathrm{~s}, 1 \mathrm{H}), 2.65$ (d, $1 \mathrm{H}, J=1.81 \mathrm{~Hz}$ ), 2.31 (ddd, $1 \mathrm{H}, J=9.49,6.11,3.33 \mathrm{~Hz}$ ), $1.70(\mathrm{~s}, 1 \mathrm{H}), 1.56(\mathrm{~s}, 1 \mathrm{H}), 1.45(\mathrm{~s}, 1 \mathrm{H}), 1.39(\mathrm{~s}, 1 \mathrm{H}), 1.15(\mathrm{~s}$, $1 \mathrm{H}), 0.82(\mathrm{t}, 1 \mathrm{H}, J=6.86 \mathrm{~Hz}), 0.71(\mathrm{t}, 1 \mathrm{H}, J=7.06 \mathrm{~Hz}) ;{ }^{13} \mathrm{C}$ NMR $\left(100.0 \mathrm{MHz}, \mathrm{CDCl}_{3}\right) \delta 179.7,179.4,125.8,134.5,128.8$, $128.7,128.4,128.3,128.2,128.1,128.1,128.0,127.8,126.6$, $126.5,125.8,123.5,78.0,75.4,75.0,53.2,49.4,47.4,41.6,32.4,31.8,29.7,29.4,28.0$, 24.3, 22.8, 22.7, 22.4, 14.4, 14.2 IR (thin film) vmax 2926, 2859, 1698, 1650, 1459, 1103, 1010, $747 \mathrm{~cm}^{-1}$; HRMS calculated for $\mathrm{C}_{30} \mathrm{H}_{38} \mathrm{O}_{3}$ : 446.2821 , found: 469.2719 $(\mathrm{M}+\mathrm{Na})$. See H-NOESY data (page S44) for complete stereochemical assignment.

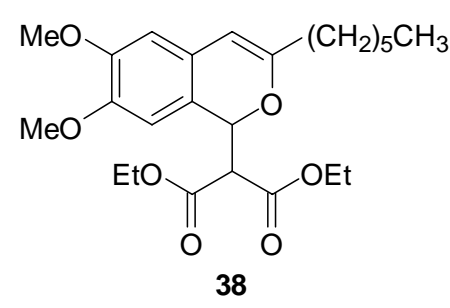

\section{Diethyl 2-(3-hexyl-6,7-dimethoxy-1H-isochromen-1-yl)- malonate (38).}

General procedure for condition a (Table 2): To a flamedried microwave reaction vessel was added alkynyl benzaldehyde 6 (20.0 mg, $0.07 \mathrm{mmol}), \mathrm{Au}(\mathrm{OAc})_{3}(2.7 \mathrm{mg}, 7$ $\mu \mathrm{mol})$, diethyl malonate $13(17 \mu \mathrm{L}, 0.11 \mathrm{mmol})$, and dichloroethane $(210 \mu \mathrm{L})$. The reaction was incubated in the microwave at $110{ }^{\circ} \mathrm{C}(300 \mathrm{~W})$ for $15 \mathrm{~min}$. The solvent was evaporated in vacuo and the crude material purified by flash chromatography $\left(\mathrm{SiO}_{2}, 30: 70 \mathrm{EtOAc} /\right.$ petroleum ether) to afford $38(62 \%, 29 \mathrm{mg}, 0.067 \mathrm{mmol})$ as a colorless oil. ${ }^{1} \mathrm{H}$ NMR $\left(400 \mathrm{MHz}, \mathrm{CDCl}_{3}\right) \delta$ $6.66(\mathrm{~s}, 1 \mathrm{H}), 6.51(\mathrm{~s}, 1 \mathrm{H}), 5.82(\mathrm{~d}, 1 \mathrm{H}, J=10.1 \mathrm{~Hz}), 5.61(\mathrm{~s}, 1 \mathrm{H}), 4.27(\mathrm{~m}, 2 \mathrm{H}), 4.08$ $(\mathrm{d}, 1 \mathrm{H}, J=10.1 \mathrm{~Hz}), 4.00(\mathrm{dddd}, 2 \mathrm{H}, J=3.7,7.1,14.4,17.9 \mathrm{~Hz}), 3.86(\mathrm{~s}, 3 \mathrm{H}), 3.82$ (s, $3 \mathrm{H}), 2.12$ (dt, $2 \mathrm{H}, J=2.7,7.1 \mathrm{~Hz}), 1.49$ (tdd, $2 \mathrm{H}, J=5.0,10.2,15.4 \mathrm{~Hz}), 1.31$ (t ovrlp, $3 \mathrm{H}, J=7.1 \mathrm{~Hz}), 1.29(\mathrm{~m}, 8 \mathrm{H}), 1.09(\mathrm{t}, 3 \mathrm{H}, J=7.1 \mathrm{~Hz}), 0.88(\mathrm{t}, 3 \mathrm{H}, J=6.9 \mathrm{~Hz}) ;{ }^{13} \mathrm{C}$ NMR (100.0 MHz, $\left.\mathrm{CDCl}_{3}\right) \delta$ 166.8. 166.6, 154.0, 149.4, 147.2, 124.1, 118.4, 109.8, 
$106.8,100.3,75.9,61.9,61.8,56.3,56.1,55.4,33.8,31.9,29.2,36.6,22.8,14.4,14.3$, 14.1; IR (thin film) vmax $\mathrm{cm}^{-1}$; HRMS calculated for $\mathrm{C}_{24} \mathrm{H}_{34} \mathrm{O}_{7}$ : 434.2305, found: $457.2125(\mathrm{M}+\mathrm{Na})$.

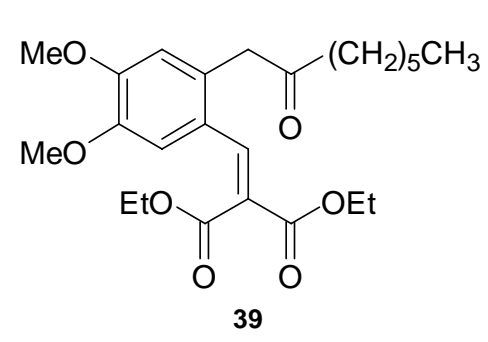

\section{Diethyl 2-(4,5-dimethoxy-2-(2-oxooctyl)benzylidene)- malonate (39).}

To a microwave reaction vessel was added alkynyl benzaldehyde 6 ( $20.0 \mathrm{mg}, 0.07 \mathrm{mmol}), \mathrm{Au}(\mathrm{OAc})_{3}(2.7 \mathrm{mg}$, $7 \mu \mathrm{mol})$, diethyl malonate $13(17 \mu \mathrm{L}, 0.11 \mathrm{mmol})$, and acetonitrile $(210 \mu \mathrm{L})$. The reaction was incubated in the microwave at $110^{\circ} \mathrm{C}(300 \mathrm{~W})$ for $15 \mathrm{~min}$. The solvent was removed and the crude material purified by flash chromatography $\left(\mathrm{SiO}_{2}, 30: 70\right.$ EtOAc/petroleum ether) to afford alkylidene malonate $39(90 \%, 29 \mathrm{mg}, 0.067 \mathrm{mmol})$ as a colorless oil. ${ }^{1} \mathrm{H}$ NMR $\left(400 \mathrm{MHz}, \mathrm{CDCl}_{3}\right) \delta 7.81(\mathrm{~s}, 1 \mathrm{H}), 7.00(\mathrm{~s}, 1 \mathrm{H}), 6.68(\mathrm{~s}, 1 \mathrm{H})$, 4.30 (q, $1 \mathrm{H}, J=7.1 \mathrm{~Hz}), 4.23(\mathrm{q}, 1 \mathrm{H}, J=7.1 \mathrm{~Hz}), 3.73(\mathrm{~s}, 2 \mathrm{H}), 2.42(\mathrm{t}, 1 \mathrm{H}, J=7.4 \mathrm{~Hz})$ 1.59 (m, $2 \mathrm{H}), 1.33$ (t ovtlp, $3 \mathrm{H}, J=7.1 \mathrm{~Hz}), 1.24$ (m ovrlp, $6 \mathrm{H}$ ), 1.20 (t ovrlp, $3 \mathrm{H}, J=$ $7.1 \mathrm{~Hz}) 0.85(\mathrm{t}, 3 \mathrm{H}, J=6.9 \mathrm{~Hz}) ;{ }^{13} \mathrm{C} \mathrm{NMR}\left(100.0 \mathrm{MHz}, \mathrm{CDCl}_{3}\right) \delta 208.1,166.9,164.2$, $150.8,148.1,140.7,128.2,127.2,125.3,113.5,111.3,61.9,61.8,56.2,56.1,47.5,42.3$, 31.8, 29.0, 23.9, 22.7, 14.4, 14.2, 14.2; ; IR (thin film) vmax $\mathrm{cm}^{-1} 2954,2931,2857$, 1720, 1600, 1572, 1515, 1464, 1405, 1248, 1273, 1204, 1108, 1066; HRMS calculated for $\mathrm{C}_{24} \mathrm{H}_{34} \mathrm{O}_{7}: 434.2305$, found: $435.2372(\mathrm{M}+\mathrm{H})$.

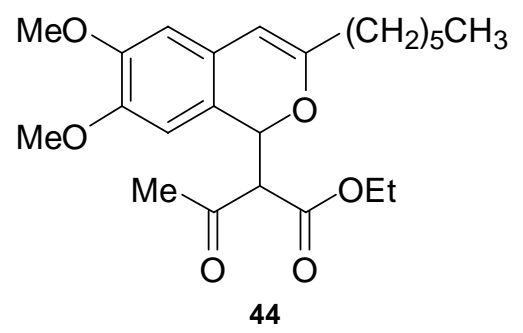

\section{Ethyl 2-(3-hexyl-6,7-dimethoxy-1H-isochromen-1-yl)- 3-oxobutanoate (44).}

General procedure for condition $b$ : To a flame-dried reaction vessel was added alkynyl benzaldehyde 6 (20.0 $\mathrm{mg}, \quad 0.07 \mathrm{mmol}), \mathrm{Rh}(\mathrm{cod})_{2} \mathrm{BF}_{4}(1.5 \mathrm{mg}, 3.6 \mu \mathrm{mol})$, and keto ester $43(14 \mathrm{mg}, 0.1 \mathrm{mmol})$. The reaction was diluted with dichloroethane $(210 \mu \mathrm{L})$ and stirred at room temperature for $4 \mathrm{~h}$. The solvent was removed and the crude material purified by flash chromatography $\left(\mathrm{SiO}_{2}, 30: 70 \mathrm{EtOAc} /\right.$ petroleum ether) to afford pure isochromane $\mathbf{4 4}$ (85 $\%, 25 \mathrm{mg}, 0.06 \mathrm{mmol})$ as a colorless oil. ${ }^{1} \mathrm{H}$ NMR $\left(400 \mathrm{MHz}, \mathrm{CDCl}_{3}, 1: 1\right.$ mixture of diastereomers). $\delta 6.55$ (s, $0.5 \mathrm{H}$ ), $6.51(\mathrm{~s}, 0.5 \mathrm{H}), 6.45$ (s ovrlp, $0.5 \mathrm{H}$ ), 6.44 (s ovrlp, 0.5 H), $5.87(\mathrm{~d}, 0.5 \mathrm{H}, J=10.1 \mathrm{~Hz}), 5.82(\mathrm{~d}, 0.5 \mathrm{H}, J=10.0 \mathrm{~Hz}), 5.53(\mathrm{~s}, 1 \mathrm{H}), 4.34(\mathrm{~d}, 0.5$ $\mathrm{H}, J=10.0 \mathrm{~Hz}), 4.25(\mathrm{dq}, 1 \mathrm{H}, J=3.5,7.1 \mathrm{~Hz}), 4.12(\mathrm{~d}, 1 \mathrm{H}, J=7.2 \mathrm{~Hz}), 4.02(\mathrm{dq}, 1 \mathrm{H}, J$ $=3.5,7.1 \mathrm{~Hz}), 3.79(\mathrm{~d}, 3 \mathrm{H}), 3.75(\mathrm{~s}, 3 \mathrm{H}), 2.30(\mathrm{~s}, 1.5 \mathrm{H}), 2.10(\mathrm{dtd}, 1 \mathrm{H}, J=3.4,7.2$, $14.4 \mathrm{~Hz}), 1.82(\mathrm{~s}, 1.5 \mathrm{H}), 1.47(\mathrm{~m}, 1 \mathrm{H}), 1.24(\mathrm{t}$ ovrlp, $1.5 \mathrm{H}, J=7.1 \mathrm{~Hz}), 1.22(\mathrm{~m}, 4 \mathrm{H})$, $1.11(\mathrm{t}, 1.5 \mathrm{H}, J=7.1 \mathrm{~Hz}), 0.88(\mathrm{t}, 1 \mathrm{H}, J=7.1 \mathrm{~Hz}) ;{ }^{13} \mathrm{C} \mathrm{NMR}\left(100.0 \mathrm{MHz}, \mathrm{CDCl}_{3}\right) \delta$ $164.2,150.8,148.1,140.6,128.1,125.3,113.5,111.4,61.8,61.8,56.2,56.1,47.4,42.3$, $31.8,29.0,23.9,22.7,14.2,14.2$; IR (thin film) vmax 2956, 2933, 2859, 1753, 1735m $1517,1458,1260,1227,1147,1116,1026 \mathrm{~cm}^{-1}$; HRMS calculated for $\mathrm{C}_{23} \mathrm{H}_{32} \mathrm{O}_{6}$ : 404.2199, found: $427.2083(\mathrm{M}+\mathrm{Na})$. 


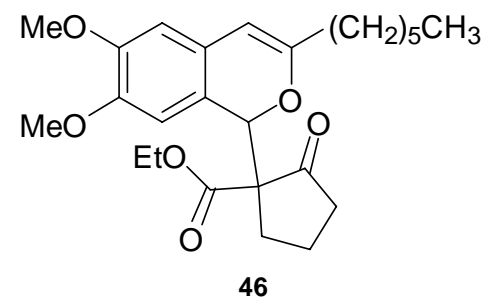

Ethyl 1-(3-hexyl-6,7-dimethoxy-1H-isochromen-1-yl)-2oxocyclopentanecarboxylate (46).

${ }^{1} \mathrm{H}$ NMR $\left(400 \mathrm{MHz}, \mathrm{CDCl}_{3}, 2: 1\right.$ mixture of diastereomers) $\delta 6.47(\mathrm{~s}, 0.33 \mathrm{H}), 6.46(\mathrm{~s}, 0.66 \mathrm{H}), 6.42(\mathrm{~s}, 0.66), 6.40(\mathrm{~s}$, $0.33 \mathrm{H}), 6.18(\mathrm{~s}, 0.66 \mathrm{H}), 5.91(\mathrm{~s}, 0.33 \mathrm{H}), 5.47(\mathrm{~s}, 0.33 \mathrm{H})$, $5.37(\mathrm{~s}, 0.66 \mathrm{H}), 4.36(\mathrm{~m}, 1 \mathrm{H}), 4.24(\mathrm{~m}$ ovrlp, $1 \mathrm{H}), 4.20(\mathrm{q}$ ovrlp, $1 \mathrm{H}, J=7.2 \mathrm{~Hz}) 3.14(\mathrm{t}, 1 \mathrm{H}, J=9.0 \mathrm{~Hz}) 2.70(\mathrm{~m}$, 1H) $0.88(\mathrm{dt}, 1 \mathrm{H}, J=1.9 \mathrm{~Hz}, J=6.8 \mathrm{~Hz}) ;{ }^{13} \mathrm{C}$ NMR $(100.0$ $\left.\mathrm{MHz}, \mathrm{CDCl}_{3}\right) \delta 200.9,200.6,167.4,167.1,154.2,153.9,149.5,149.4,147.4,147.4$, 124.1, 118.5, 118.4, 109.9, 109.1, 107.0, 106.9, 105.0, 100.4, 100.1, 75.9, 63.4, 61.9, 56.4, 56.1, 52.8, 52.7, 52.6, 50.1, 33.8, 33.7, 32.2, 31.9, 31.8, 30.4, 29.2, 29.1, 28.8, 26.7, 26.6, 22.8, 14.3; IR (thin film) vmax 2956, 2933, 2859, 1753, 1725, 1517, 1458, 1260, 1227, 1147, 1116, $1026 \mathrm{~cm}^{-1}$; HRMS calculated for $\mathrm{C}_{25} \mathrm{H}_{34} \mathrm{O}_{6}$ : 430.2355, found: $453.2200(\mathrm{M}+\mathrm{Na})$.

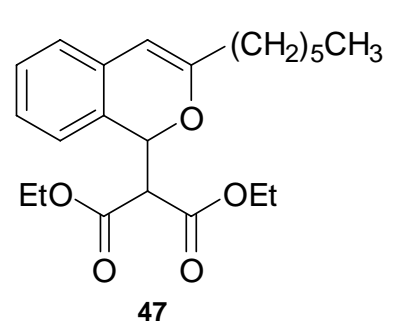

Diethyl 2-(3-hexyl-1H-isochromen-1-yl)malonate (47).

${ }^{1} \mathrm{H}$ NMR $\left(400 \mathrm{MHz}, \mathrm{CDCl}_{3}\right) \delta 4.27(\mathrm{~m}, 2 \mathrm{H}), 4.11(\mathrm{~d}, 1 \mathrm{H}, J=$ $10.09 \mathrm{~Hz}$ ), 3.99 (dddd, $2 \mathrm{H}, J=17.91,10.78,7.14,3.64 \mathrm{~Hz}$ ), $2.14(\mathrm{dt}, 2 \mathrm{H}, J=7.23,7.21,3.17 \mathrm{~Hz}), 1.50(\mathrm{~m}, 2 \mathrm{H}), 1.30(\mathrm{t}$ obsc, $3 \mathrm{H}), 1.29(\mathrm{~m}, 8 \mathrm{H}), 1.07(\mathrm{t}, 3 \mathrm{H}, J=4.78 \mathrm{~Hz}), 0.88(\mathrm{t}, 3 \mathrm{H}$, $J=6.79 \mathrm{~Hz}),{ }^{13} \mathrm{C} \mathrm{NMR}\left(100.0 \mathrm{MHz}, \mathrm{CDCl}_{3}\right) \delta 166.8,166.3$, $155.7,130.9,128.9,126.4,125.9,125.8,123.3,100.8,76.0$, $61.9,61.7,55.3,33.9,31.8,29.2,26.6,22.8,14.4,14.3,14.0$; IR (thin film) vmax 2956, $2930,1735,1459,1371,1256,1156,1030 \mathrm{~cm}^{-1}$; HRMS calculated for $\mathrm{C}_{22} \mathrm{H}_{30} \mathrm{O}_{5}$ : 374.2093, found: $397.1991(\mathrm{M}+\mathrm{Na})$.

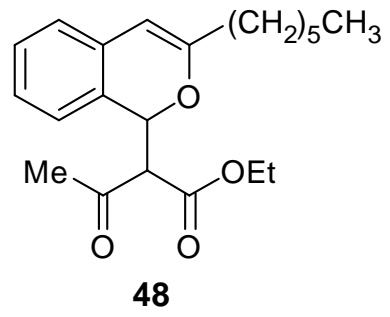

Ethyl 2-(3-hexyl-1H-isochromen-1-yl)-3-oxobutanoate (48).

${ }^{1} \mathrm{H}$ NMR (400 MHz, $\mathrm{CDCl}_{3}, 1: 1$ mixture of diastereomers) $\delta 7.20$ (ddt, $1 \mathrm{H}, J=7.49,3.63,1.38 \mathrm{~Hz}$ ), 7.07 (ddt, $1 \mathrm{H}, J=7.47,3.65$, $1.21 \mathrm{~Hz}), 7.10(\mathrm{~m}, 2 \mathrm{H}), 5.91(\mathrm{~d}, 0.5 \mathrm{H}, J=10.16 \mathrm{~Hz}), 5.88(\mathrm{~d}$, $0.5 \mathrm{H}, J=10.02 \mathrm{~Hz}), 5.7(\mathrm{~s}, 1 \mathrm{H}) 4.25(\mathrm{qd}, 1 \mathrm{H}, J=6.10,3.69$ $\mathrm{Hz}), 4.19(\mathrm{~d}, 0.5 \mathrm{H}, J=10.08 \mathrm{~Hz}), 4.01(\mathrm{dq}, 1 \mathrm{H}, J=7.15,1.57$ $\mathrm{Hz}), 2.37(\mathrm{~s}, 1.5 \mathrm{H}), 2.17-2.06(\mathrm{~m}, 2 \mathrm{H}), 1.86(\mathrm{~s}, 1.5 \mathrm{H}), 1.53-$ $1.39(\mathrm{~m}, 2 \mathrm{H}), 1.36$ (t obsc, $1.5 \mathrm{H}), 1.36-1.23(\mathrm{~m}, 8 \mathrm{H}), 1.09(\mathrm{t}, 1.5 \mathrm{H}, J=7.14 \mathrm{~Hz}), 0.88$ $(\mathrm{t}, 3 \mathrm{H}, J=6.82 \mathrm{~Hz}) ;{ }^{13} \mathrm{C}$ NMR $\left(100.0 \mathrm{MHz}, \mathrm{CDCl}_{3}\right) \delta 166.8,156.0,155.6,130.9,130.8$, $128.9,126.4,126.1,126.0,125.2,123.4,123.4,100.9,100.6,63.3,62.0,61.9,61.7,33.9$, $33.8,31.8,31.8,29.2,29.1,28.8,26.7,26.6,22.8,14.4,14.3,14.1$; IR (thin film) vmax $3443,2056,2930,2859,2736,1719,1459,1370,1256,1159,1027 \mathrm{~cm}^{-1}$; HRMS calculated for $\mathrm{C}_{21} \mathrm{H}_{28} \mathrm{O}_{4}$ : 344.1988 , found: $367.2035(\mathrm{M}+\mathrm{Na})$. 


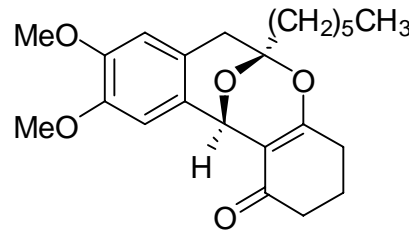

53

\section{Polycyclic ketal (53).}

To a flame-dried reaction vessel was added alkynyl benzaldehyde 6 ( $77 \mathrm{mg}, 0.28 \mathrm{mmol}), \mathrm{Rh}(\mathrm{cod})_{2} \mathrm{BF}_{4}(5.7 \mathrm{mg}, 14$ $\mu \mathrm{mol})$, and 1,3-cylcohexanedione 52 (47 $\mathrm{mg}, 0.42 \mathrm{mmol})$. The reaction was diluted with dichloroethane $(800 \mu \mathrm{L})$ and stirred at room temperature for $4 \mathrm{~h}$. The solvent was removed in vacuo and the crude material urified by flash chromatography $\left(\mathrm{SiO}_{2}, 30: 70 \mathrm{EtOAc} /\right.$ petroleum ether) to afford polycyclic ketal $53(68 \%, 74 \mathrm{mg}, 0.19$ mmol) as a colorless oil. m.p. $=59-60{ }^{\circ} \mathrm{C} ;{ }^{1} \mathrm{H}$ NMR $\left(400 \mathrm{MHz}, \mathrm{CDCl}_{3}\right) \delta 6.98(\mathrm{~s}, 1 \mathrm{H})$, $6.58(\mathrm{~s}, 1 \mathrm{H}), 5.66(\mathrm{~s}, 1 \mathrm{H}), 3.87(\mathrm{~s}, 3 \mathrm{H}), 3.82$ (s, $3 \mathrm{H}), 3.07$ (dd, $2 \mathrm{H}, J=115.82,17.90$ $\mathrm{Hz}$ ), 2.41-2.22 (m, $4 \mathrm{H}$ ), 1.94 (dddd, $4 \mathrm{H}, J=14.35,13.14,10.77,9.54 \mathrm{~Hz}$ ), 1.61-1.42 $(\mathrm{m}, 2 \mathrm{H}), 1.41-1.21(\mathrm{~m}, 6 \mathrm{H}), 0.89(\mathrm{t}, 3 \mathrm{H}, J=6.19 \mathrm{~Hz}) ;{ }^{13} \mathrm{C} \mathrm{NMR}\left(100.0 \mathrm{MHz}, \mathrm{CDCl}_{3}\right) \delta$ $194.9,168.7,148.3,147.5,130.2,121.2,114.9,110.9,108.8,102.9,67.1,56.2,56.2$, $41.0,38.2,36.6,31.9,29.5,28.0,22.8,22.7,20.9,14.3$; IR (thin film) vmax 2945, 2863, $1627,1513,1459,1392,1261,1234,1133,1000 \mathrm{~cm}^{-1}$; HRMS calculated for $\mathrm{C}_{23} \mathrm{H}_{30} \mathrm{O}_{5}$ : 386.2093, found: $387.2101(\mathrm{M}+\mathrm{H})$.

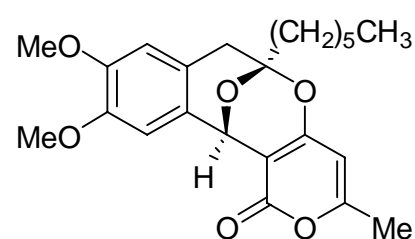

57

Polycyclic ketal (57).

m.p. $=110-115{ }^{\circ} \mathrm{C} ;{ }^{1} \mathrm{H}$ NMR $\left(400 \mathrm{MHz}, \mathrm{CDCl}_{3}\right) \delta 7.02$ (s, 1 $\mathrm{H}), 6.59(\mathrm{~s}, 1 \mathrm{H}), 5.76(\mathrm{~s}, 1 \mathrm{H}), 5.70(\mathrm{~s}, 1 \mathrm{H}), 3.88(\mathrm{~s}, 3 \mathrm{H}), 3.82$ (s, $3 \mathrm{H}), 3.11$ (dd, $2 \mathrm{H}, J=121.18,18.10 \mathrm{~Hz}), 2.15(\mathrm{~s}, 3 \mathrm{H})$, 1.97 (ddd, $2 \mathrm{H}, J=12.50,10.61,5.95 \mathrm{~Hz}), 1.53(\mathrm{~m}, 2 \mathrm{H}), 1.33$ $(\mathrm{m}, 6 \mathrm{H}), 0.89(\mathrm{t}, 3 \mathrm{H}, J=6.91 \mathrm{~Hz}) ;{ }^{13} \mathrm{C} \mathrm{NMR}(100.0 \mathrm{MHz}$, $\left.\mathrm{CDCl}_{3}\right) \delta 162.2,162.0,161.9,148.6,147.8,129.0,121.0$, 111.1, 108.4, 103.6, 101.2, 100.0, 67.4, 56.3, 56.2, 40.9, 38.0, 31.9, 29.5, 22.8, 22.6, 20.2, 14.3; IR (thin film) vmax 2929, 2859, 1716, 1650, 1514, 1458, 1234, 1108, $989 \mathrm{~cm}^{-1}$; HRMS calculated for $\mathrm{C}_{23} \mathrm{H}_{38} \mathrm{O}_{6}$ : 400.1886 , found: $401.1964(\mathrm{M}+\mathrm{H})$.

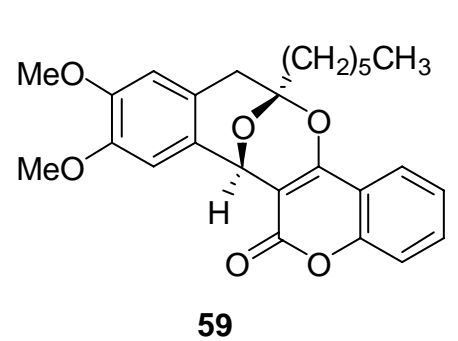

\section{Polycyclic ketal (59).}

${ }^{1} \mathrm{H}$ NMR $\left(400 \mathrm{MHz}, \mathrm{CDCl}_{3}\right) \delta 7.74(\mathrm{dd}, 1 \mathrm{H}, J=7.81,1.61$ $\mathrm{Hz}), 7.58-7.38(\mathrm{~m}, 1 \mathrm{H}), 7.32-7.22(\mathrm{~m}, 2 \mathrm{H}), 7.09(\mathrm{~s}, 1 \mathrm{H})$, $6.61(\mathrm{~s}, 1 \mathrm{H}), 5.85(\mathrm{~s}, 1 \mathrm{H}), 3.90(\mathrm{~s}, 3 \mathrm{H}), 3.82(\mathrm{~s}, 3 \mathrm{H}), 3.23$ $(\mathrm{dd}, 2 \mathrm{H}, J=106.33,17.99 \mathrm{~Hz}), 2.37-1.80(\mathrm{~m}, 2 \mathrm{H}), 1.75-$ $1.56(\mathrm{~m}, 2 \mathrm{H}), 1.45-1.38(\mathrm{~m}, 2 \mathrm{H}), 1.38-1.27(\mathrm{~m}, 4 \mathrm{H}), 0.90$ $(\mathrm{t}, 3 \mathrm{H}, J=6.95 \mathrm{~Hz}) ;{ }^{13} \mathrm{C} \mathrm{NMR}\left(100.0 \mathrm{MHz}, \mathrm{CDCl}_{3}\right) \delta$ $160.5,157.6,153.1,148.8,147.9,132.1,128.5,124.1,122.5$, $121.1,117.1,115.4,111.1,108.6,104.3,104.0,67.7,56.3,56.2,40.9,38.2,31.9,29.5$, 22.8, 22.7, 14.3; IR (thin film) vmax 2954, 2931, 2857, 2360, 2339, 1720, 1600, 1515, $1464,1273,1248,1204,1108,1066 \mathrm{~cm}^{-1}$; HRMS calculated for $\mathrm{C}_{26} \mathrm{H}_{28} \mathrm{O}_{6}: 436.1886$, found: $459.2359(\mathrm{M}+\mathrm{Na})$. 


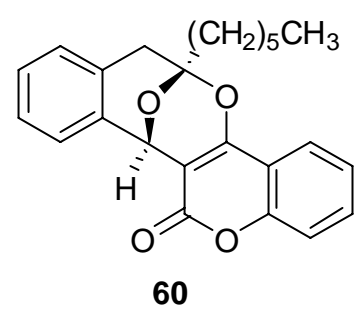

Polycyclic ketal (60).

${ }^{1} \mathrm{H}$ NMR $\left(400 \mathrm{MHz}, \mathrm{CDCl}_{3}\right) \delta 7.75(\mathrm{dd}, 1 \mathrm{H}, J=7.76,1.61 \mathrm{~Hz})$, $7.59(\mathrm{dd}, 1 \mathrm{H}, J=6.13,2.85 \mathrm{~Hz}), 7.51(\mathrm{ddd}, 1 \mathrm{H}, J=8.57,7.01$, $1.47 \mathrm{~Hz}), 7.27(\mathrm{~m}, 4 \mathrm{H}), 7.21(\mathrm{dd}, 1 \mathrm{H}, J=5.65,3.30 \mathrm{~Hz}), 7.18(\mathrm{~m}$, $2 \mathrm{H}), 5.92(\mathrm{~s}, 1 \mathrm{H}), 3.30(\mathrm{dd}, 1 \mathrm{H}, J=110.00,18.36 \mathrm{~Hz}), 2.24-2.02$ $(\mathrm{m}, 2 \mathrm{H}), 1.73-1.57(\mathrm{~m}, 2 \mathrm{H}), 1.44-1.25(\mathrm{~m}, 6 \mathrm{H}), 0.90(\mathrm{t}, 3 \mathrm{H}, J=$ $7.02 \mathrm{~Hz}) ;{ }^{13} \mathrm{C}$ NMR $\left(100.0 \mathrm{MHz}, \mathrm{CDCl}_{3}\right) \delta 160.3,157.5,153.2$, $136.2,132.2$, 128.1, 127.9, 125.8, 124.1, 122.6, 117.1, 104.2, 103.5, 68.0, 40.9, 38.6, $31.9,31.5,29.5,28.9,28.7,22.8,22.7,19.8,14.3$; IR (thin film) vmax 2931, 2859, 1710, $1635,1513,1458,1403,1262,1233,1114,1032,758 \mathrm{~cm}^{-1}$ HRMS calculated for $\mathrm{C}_{24} \mathrm{H}_{24} \mathrm{O}_{4}: 376.1675$, found: $393.2061(\mathrm{M}+\mathrm{Na})$.

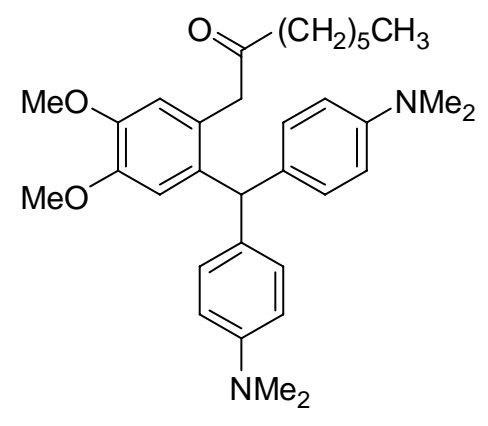

62

4-(1-(4-(dimethylamino)phenyl)-3-hexyl-6,7-dimethoxy1H-isochromen-1-yl)- $\mathrm{N}, \mathrm{N}$-dimethylbenzenamine (62).

To a flame-dried reaction vessel was added benzaldehyde $\mathbf{6}$ (20 mg, $73 \mu \mathrm{mol})$, dimethyl aniline $24(10 \mathrm{mg}, 80 \mu \mathrm{mol})$, and $\mathrm{C}_{2} \mathrm{H}_{4} \mathrm{Cl}_{2}$. Into the reaction was added $\mathrm{AgSbF}_{6}(25 \mathrm{mg}$, $73 \mu \mathrm{mol})$ and the reaction immediately turned dark purple. The reaction was stirred for $24 \mathrm{~h}$ at $\mathrm{rt}$. The solution was filtered through a $\mathrm{SiO}_{2}$ plug eluting with EtOAc. The solvent was removed in vacuo and the crude material was purified by flash chromatography $\left(\mathrm{SiO}_{2}, \quad 40: 60\right.$ EtOAc/petroleum ether) to afford bis-aniline $62(48 \%, 18 \mathrm{mg}, 37 \mu \mathrm{mol})$ as a yellowish oil. ${ }^{1} \mathrm{H} \mathrm{NMR}\left(400 \mathrm{MHz}, \mathrm{CDCl}_{3}\right) \delta 6.89(\mathrm{~d}, J=8.62 \mathrm{~Hz}, 1 \mathrm{H}), 6.64(\mathrm{~s}, 1 \mathrm{H}), 6.46(\mathrm{~s}, 1 \mathrm{H})$, $5.35(\mathrm{~s}, 1 \mathrm{H}), 3.84(\mathrm{~s}, 1 \mathrm{H}), 3.63(\mathrm{~s}, 1 \mathrm{H}), 3.58(\mathrm{~s}, 1 \mathrm{H}), 2.91(\mathrm{~s}, 1 \mathrm{H}), 2.16(\mathrm{t}, J=7.51,7.51$ $\mathrm{Hz}, 1 \mathrm{H}), 1.41(\mathrm{~s}, 1 \mathrm{H}), 1.21(\mathrm{~s}, 1 \mathrm{H}), 0.86(\mathrm{t}, J=7.08,7.08 \mathrm{~Hz}, 1 \mathrm{H}) ;\left(100.0 \mathrm{MHz}, \mathrm{CDCl}_{3}\right)$ $\delta 208.5,166.7,164.2,150.3,148.1,140.2,132.0,131.1,130.3,127.1,126.4,112.8,48.7$, 42.2, 41.3, 31.8, 29.1, 24.2, 22.3, 14.4 IR (thin film) vmax 2954, 2931, 2857, 1720, 1600, $1572,1515,1464,1405,1248,1273,1204,1066 \mathrm{~cm}^{-1}$; HRMS calculated for $\mathrm{C}_{33} \mathrm{H}_{42} \mathrm{~N}_{2} \mathrm{O}_{3}$ : 516.3352, found: $517.3425(\mathrm{M}+\mathrm{H})$.

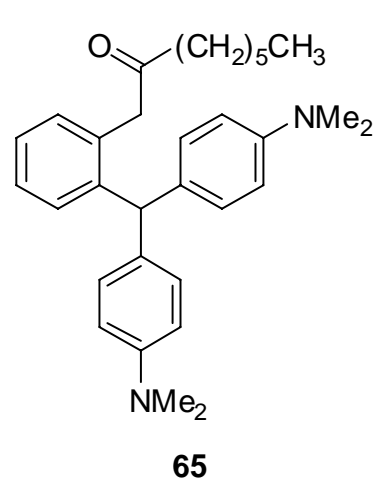

\section{4-(1-(4-(dimethylamino)phenyl)-3-hexyl-6,7-dimethoxy-1H-} isochromen-1-yl)-N,N-dimethylbenzenamine (65).

${ }^{1} \mathrm{H}$ NMR $\left(400 \mathrm{MHz}, \mathrm{CDCl}_{3}\right) \delta 6.89(\mathrm{~d}, J=8.62 \mathrm{~Hz}, 1 \mathrm{H}), 6.64(\mathrm{~s}$, $1 \mathrm{H}), 6.46(\mathrm{~s}, 1 \mathrm{H}), 5.35(\mathrm{~s}, 1 \mathrm{H}), 3.84(\mathrm{~s}, 1 \mathrm{H}), 3.63(\mathrm{~s}, 1 \mathrm{H}), 3.58$ (s, $1 \mathrm{H}), 2.91(\mathrm{~s}, 1 \mathrm{H}), 2.16(\mathrm{t}, J=7.51,7.51 \mathrm{~Hz}, 1 \mathrm{H}), 1.41(\mathrm{~s}, 1$ H), $1.21(\mathrm{~s}, 1 \mathrm{H}), 0.86(\mathrm{t}, J=7.08,7.08 \mathrm{~Hz}, 1 \mathrm{H}) ;(100.0 \mathrm{MHz}$, $\left.\mathrm{CDCl}_{3}\right) \delta 209.2,149.2,144.2,133.5,132.0,131.1,130.3,127.1$, 126.4, 112.8, 51.7, 47.7, 42.4, 40.9, 31.8, 29.1, 24.0, 22.7, 14.3; IR (thin film) 2960, 2754, 1760, 1601, 1572, 1522, 1238, 1275, 1209, 1108,vmax cm $\mathrm{cm}^{-1}$; HRMS calculated for $\mathrm{C}_{31} \mathrm{H}_{38} \mathrm{~N}_{2} \mathrm{O}$ : 456.3141, found: $457.3323(\mathrm{M}+\mathrm{H})$. 


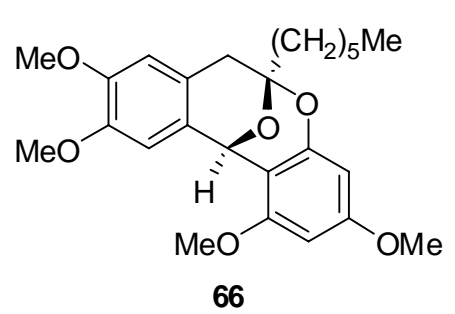

\section{Polycyclic ketal (66).}

Into a microwave reaction vessel was added aldehyde 6 (33.5 $\mathrm{mg}, 0.122 \mathrm{mmol})$, phenol 25 (37.8 $\mathrm{mg}, 0.244 \mathrm{mmol}), \mathrm{PtCl}_{2}$ $(6.4 \mathrm{mg}, 0.024 \mathrm{mmol})$, and dichloroethane $(1.5 \mathrm{~mL})$. The reaction mixture was incubated in the microwave for $3 \mathrm{~h}(300$ $\left.\mathrm{W}, 150{ }^{\circ} \mathrm{C}\right)$. the solvent was evaporated and the crude material was flash chromatographed $\left(\mathrm{SiO}_{2} \quad 0-50 \quad \%\right.$ EtOAc/hexanes) to afford pure polycyclic ketal $66(58 \%, 30.5 \mathrm{mg})$ as a yellow oil. ${ }^{1} \mathrm{H}$ NMR (400 MHz, CDCl $) \delta 6.94(\mathrm{~s}, 1 \mathrm{H}), 6.59(\mathrm{~s}, 1 \mathrm{H}), 6.01(\mathrm{dd}, 2 \mathrm{H}, J=2.2,11.9 \mathrm{~Hz})$, $5.88(\mathrm{~s}, 1 \mathrm{H}), 3.87(\mathrm{~s}, 3 \mathrm{H}), 3.82(\mathrm{~s}, 3 \mathrm{H}), 3.81(\mathrm{~s}, 3 \mathrm{H}), 3.72(\mathrm{~s}, 3 \mathrm{H}), 3.23(\mathrm{~d}, 1 \mathrm{H}, J=$ $17.7 \mathrm{~Hz}), 2.94(\mathrm{~d}, 1 \mathrm{H}, J=17.7 \mathrm{~Hz}), 1.97(\mathrm{~m}, 2 \mathrm{H}), 1.57(\mathrm{~m}, 2 \mathrm{H}), 1.32(\mathrm{~m}, 6 \mathrm{H}), 0.89$ (t, $3 \mathrm{H}, J=6.7 \mathrm{~Hz}) ;{ }^{13} \mathrm{C}$ NMR $\left(100 \mathrm{MHz}, \mathrm{CDCl}_{3}\right) \delta 160.4,156.0,152.0,148.2,147.2$, 131.0, 123.1, 111.2, 108.5, 105.9, 100.2, 93.5, 91.5, 67.9, 56.1, 55.6, 55.5, 41.7, 38.4, 32.0, 29.7, 22.8, 22.8, 14.3; IR (thin film) vmax 2997, 2952, 2856, 1619, 1597, 1513, $1499,1465,1453,1353,1325,1285,1263,1252,1229,1020,1146,1111,1049,999$, 957, 811, 776, 754, 633, $568 \mathrm{~cm}^{-1}$; HRMS calculated for $\mathrm{C}_{25} \mathrm{H}_{33} \mathrm{O}_{6}: 428.2199$, found: 429.2292 $(\mathrm{M}+\mathrm{H})$. See HMQC and HMBC data (pages S45 and S46) for structure assignment. 


\section{Computational Structure Elucidation}

NMR data $\left({ }^{1} \mathrm{H},{ }^{13} \mathrm{C},{ }^{1} \mathrm{H}-\mathrm{COSY}\right.$, HMQC, HMBC) were added to ACD Structure Elucidator program by manually inputting chemical shift and correlation data from processed spectra. Molecular formulas were generated based on the low resolution mass spectral data. A molecular connectivity diagram (MCD) was generated by the software based on the correlation data from 2-D NMR spectra ( $\left.{ }^{1} \mathrm{H}-\mathrm{COSY}, \mathrm{HMQC}, \mathrm{HMBC}\right)$. The MCD was submitted to the Structure Elucidator which generated a list of candidate structures. Duplicate molecules were removed and the resulting list of structures ranked in order of their deviation from the predicted chemical shift of the ${ }^{13} \mathrm{C}$ spectrum. The NMR spectra and elucidation data for homodimer $\mathbf{2 7}$ and tetracyclic ketal $\mathbf{5 3}$ are outlined below (S22 - S35). 


\section{ACD Structure Elucidation for homodimer 27}

${ }^{1} \mathrm{H} \mathrm{NMR}\left(400 \mathrm{MHz}, \mathrm{CDCl}_{3}\right)$
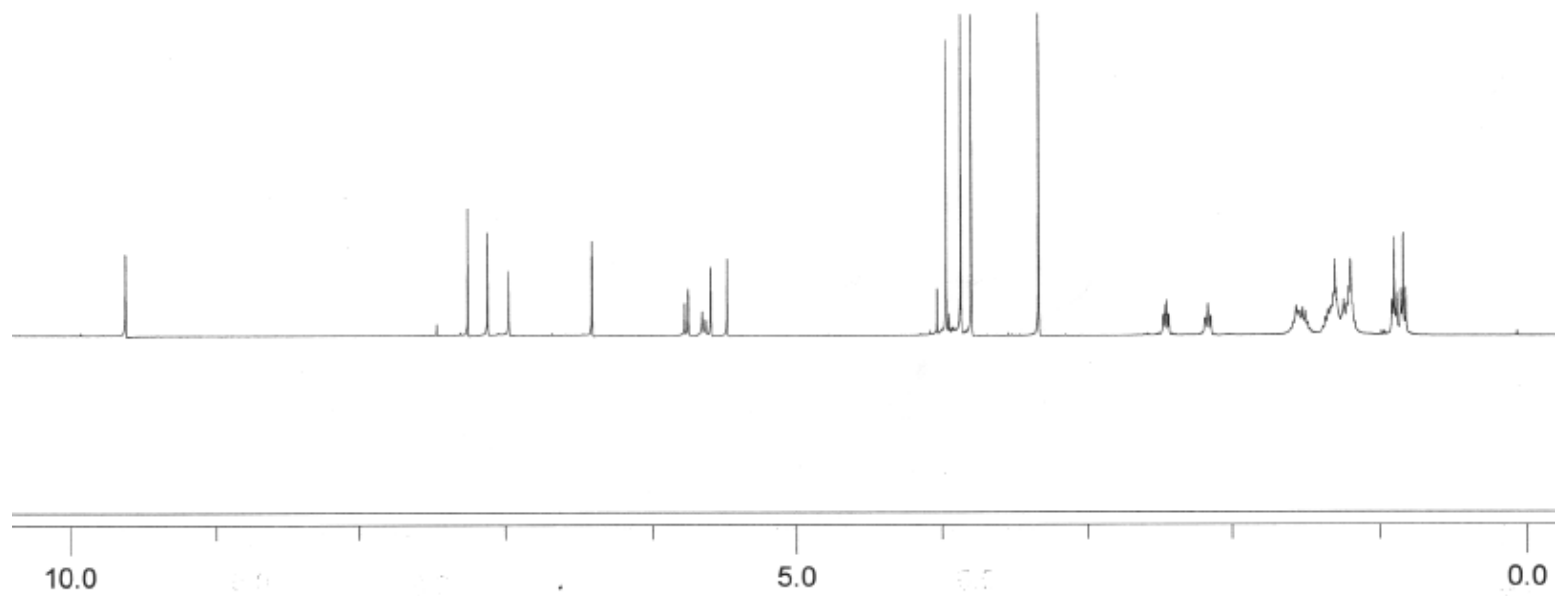

${ }^{13} \mathrm{C}$ NMR $\left(100 \mathrm{MHz}, \mathrm{CDCl}_{3}\right)$
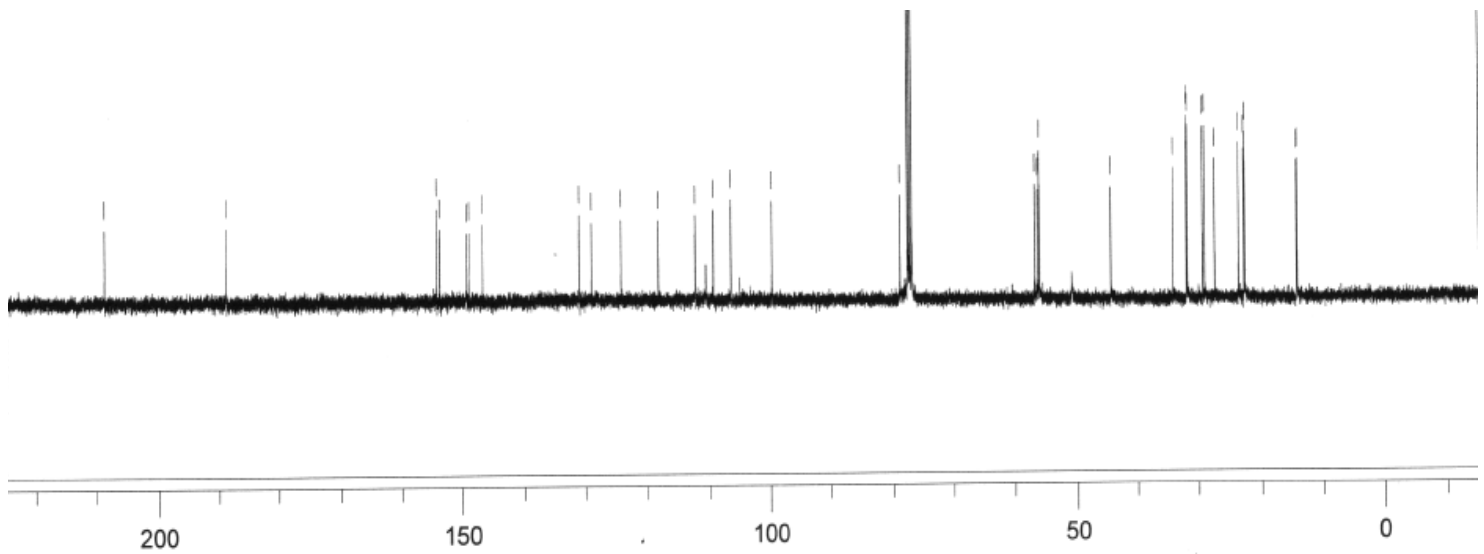

ppm (f1) 


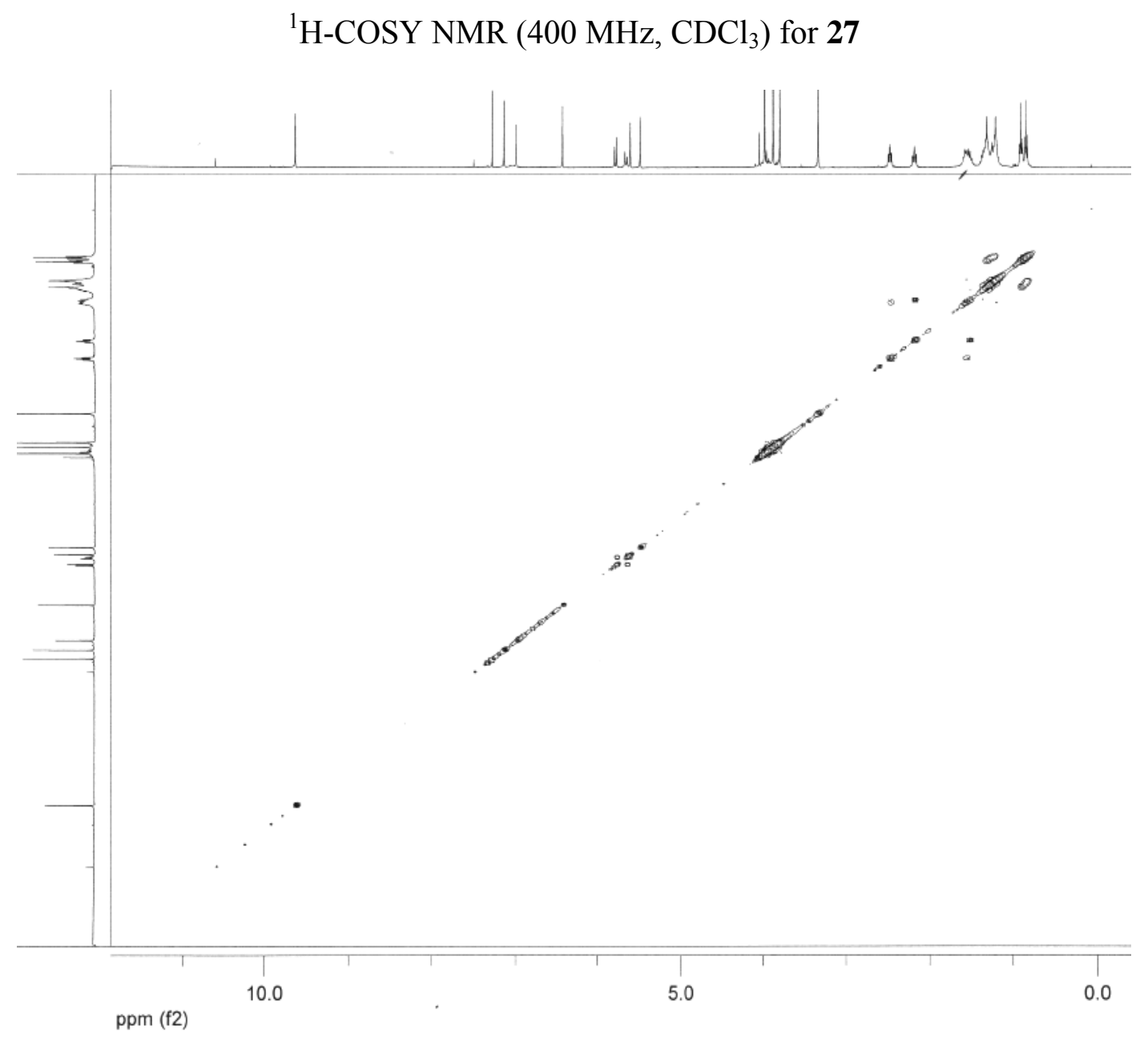


HMQC NMR (100 MHz, $\mathrm{CDCl}_{3}$ ) for 27

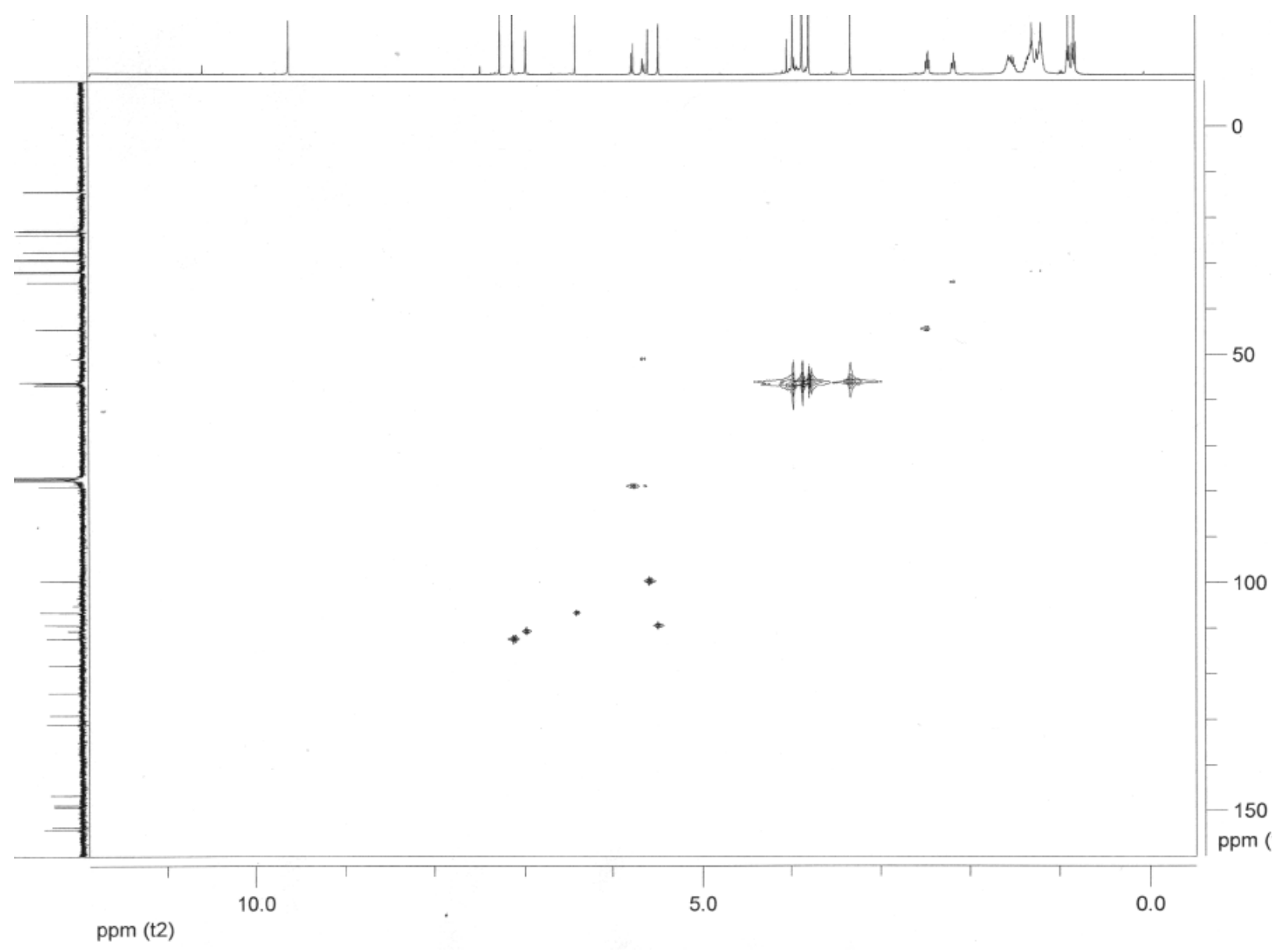


HMBC NMR (100 MHz, $\left.\mathrm{CDCl}_{3}\right)$ for 27

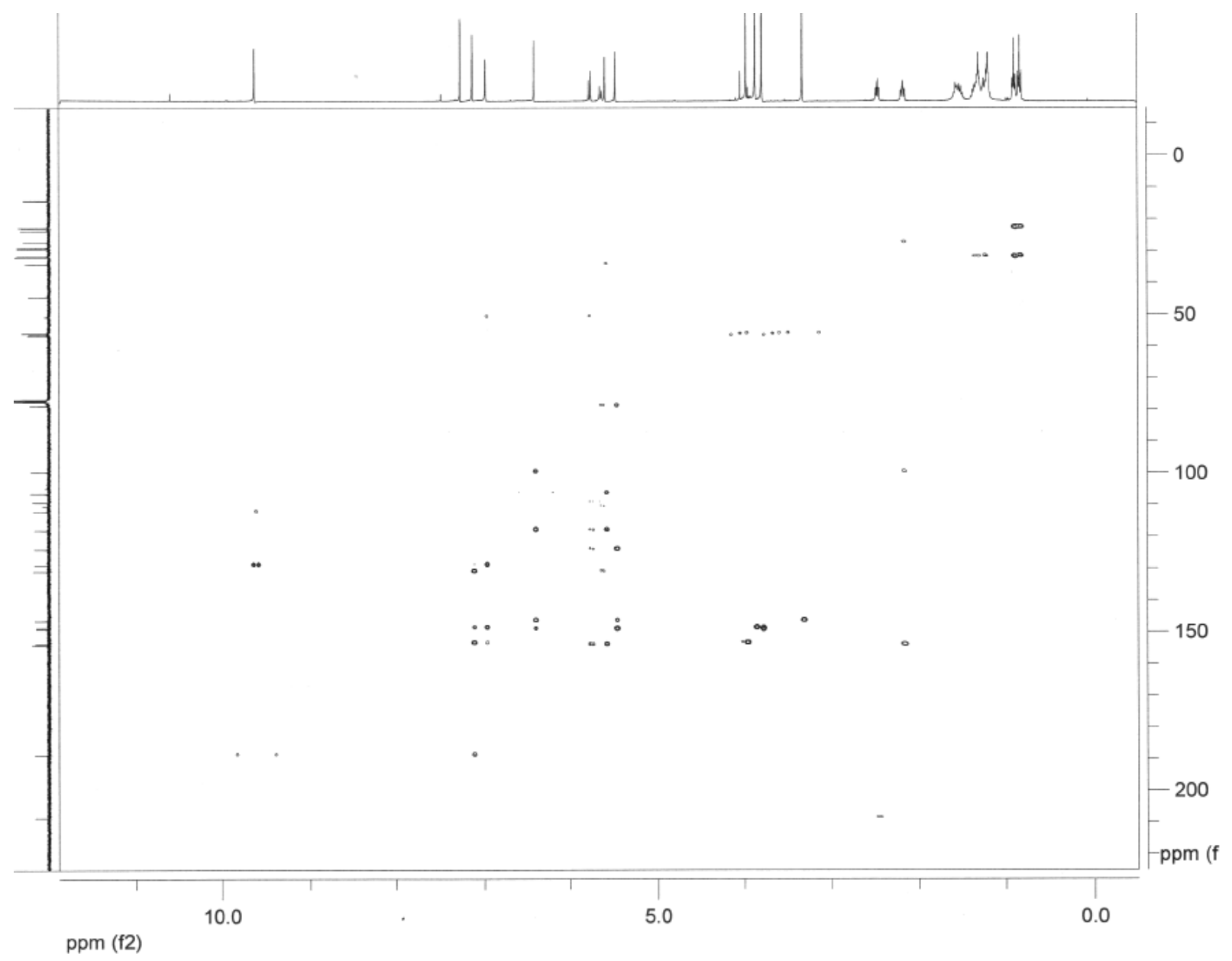


Structure Elucidator Data for $\mathbf{2 7}$

Initial Data

Composition Restrictions:

Molecular Weight $=566.324341$

Double Bonds Equivalent $=12$

Allowed Composition $=\mathrm{C}(34) \mathrm{H}(46) \mathrm{O}(7)$

Molecular Formula $=\mathrm{C} 34 \mathrm{H} 46 \mathrm{O} 7$

Spectral Data:

standard $1 \mathrm{H}$ (exp) - 19 peaks

standard $1 \mathrm{H}$ (user) -22 peaks

standard $13 \mathrm{C}$ (exp) - 34 peaks

COSY $1 \mathrm{H}-1 \mathrm{H}$ (exp) -8 peaks

HMQC $13 \mathrm{C}-1 \mathrm{H}$ (exp) - 22 peaks

HMBC $13 \mathrm{C}-1 \mathrm{H}$ (exp) -49 peaks

HMBC $13 \mathrm{C}-1 \mathrm{H}$ (exp) - 55 peaks

\section{Most Probable Structure}

Following structure was placed to the first position after spectra calculation

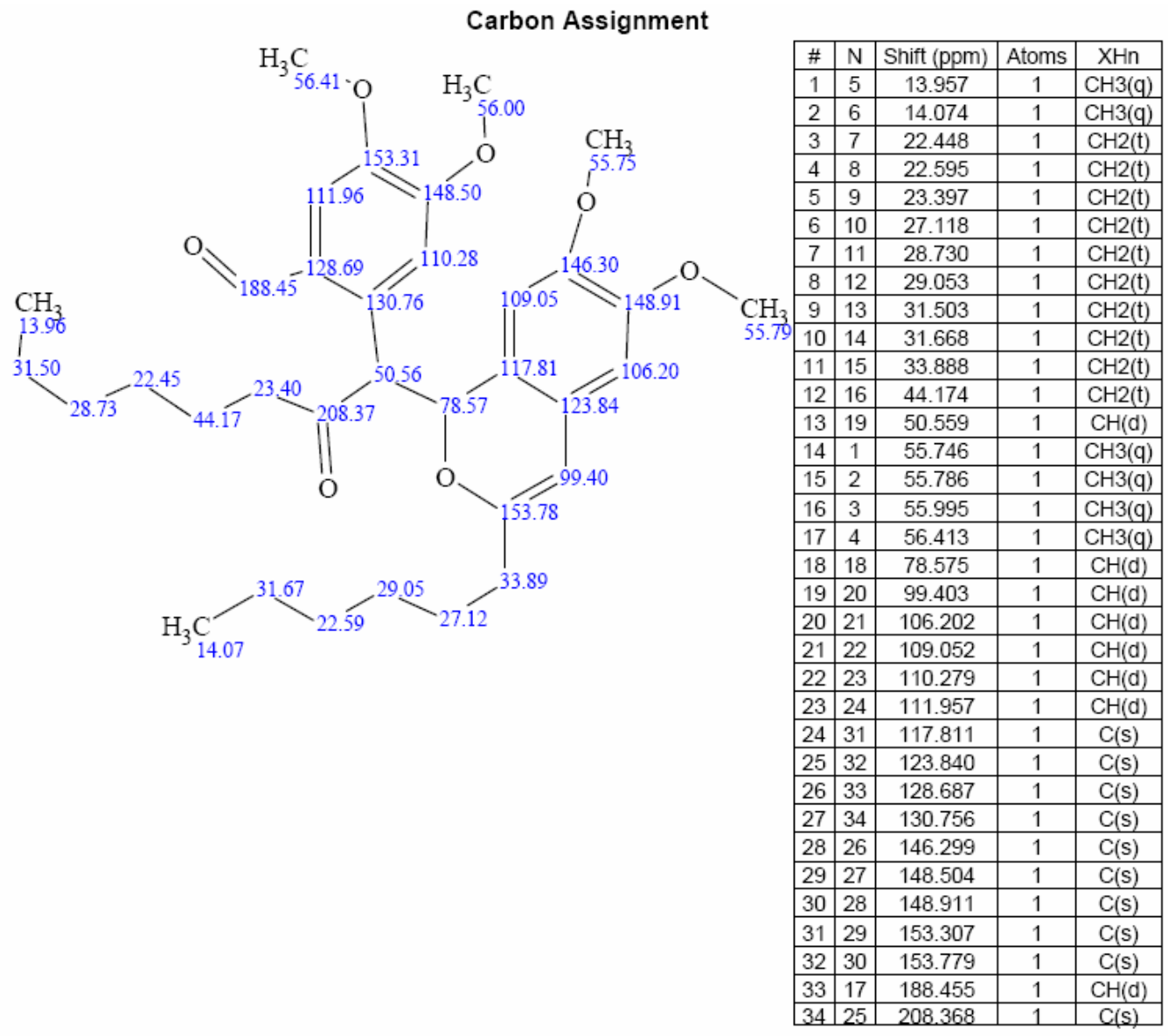




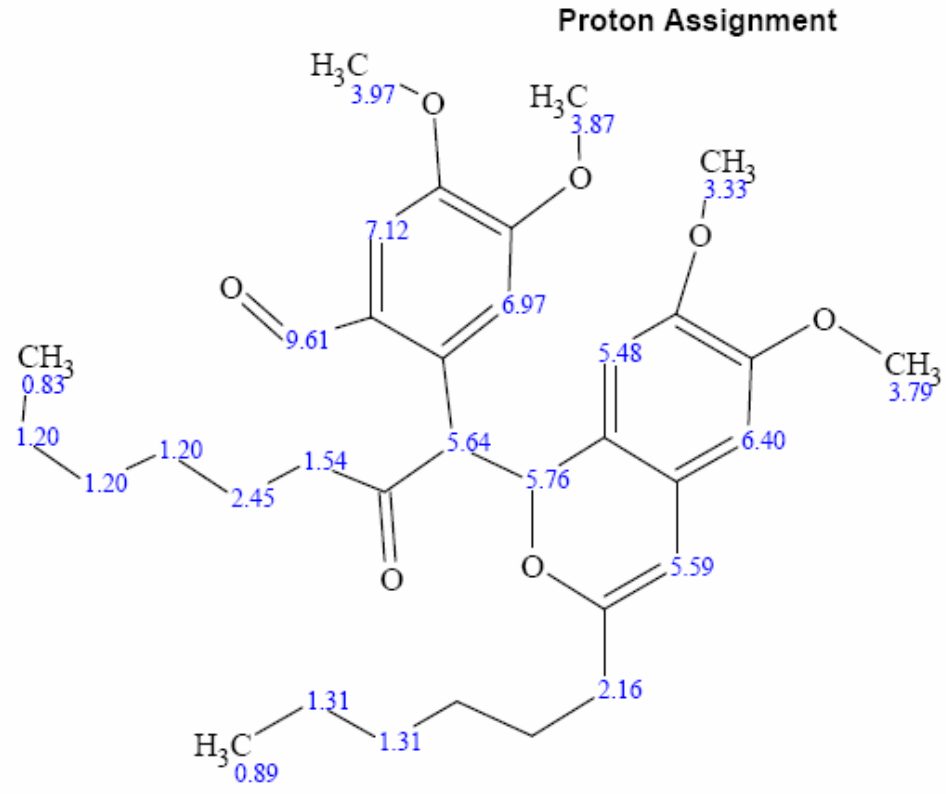

\begin{tabular}{|c|c|c|c|}
\hline$\#$ & $\mathrm{~N}$ & Shift (ppm) & Protons \\
\hline 1 & 5 & 0.832 & 3 \\
\hline 2 & 6 & 0.895 & 3 \\
\hline 3 & 11 & 1.200 & 2 \\
\hline 4 & 7 & 1.200 & 2 \\
\hline 5 & 13 & 1.201 & 2 \\
\hline 6 & 8 & 1.308 & 2 \\
\hline 7 & 14 & 1.309 & 2 \\
\hline 8 & 9 & 1.545 & 2 \\
\hline 9 & 15 & 2.163 & 2 \\
\hline 10 & 16 & 2.453 & 2 \\
\hline 11 & 1 & 3.332 & 3 \\
\hline 12 & 2 & 3.793 & 3 \\
\hline 13 & 3 & 3.867 & 3 \\
\hline 14 & 4 & 3.968 & 3 \\
\hline 15 & 22 & 5.477 & 1 \\
\hline 16 & 20 & 5.593 & 1 \\
\hline 17 & 19 & 5.639 & 1 \\
\hline 18 & 18 & 5.762 & 1 \\
\hline 19 & 21 & 6.402 & 1 \\
\hline 20 & 23 & 6.972 & 1 \\
\hline 21 & 24 & 7.122 & 1 \\
\hline 22 & 17 & 9.610 & 1 \\
\hline
\end{tabular}

Molecular Connectivity Diagrams (MCDs)

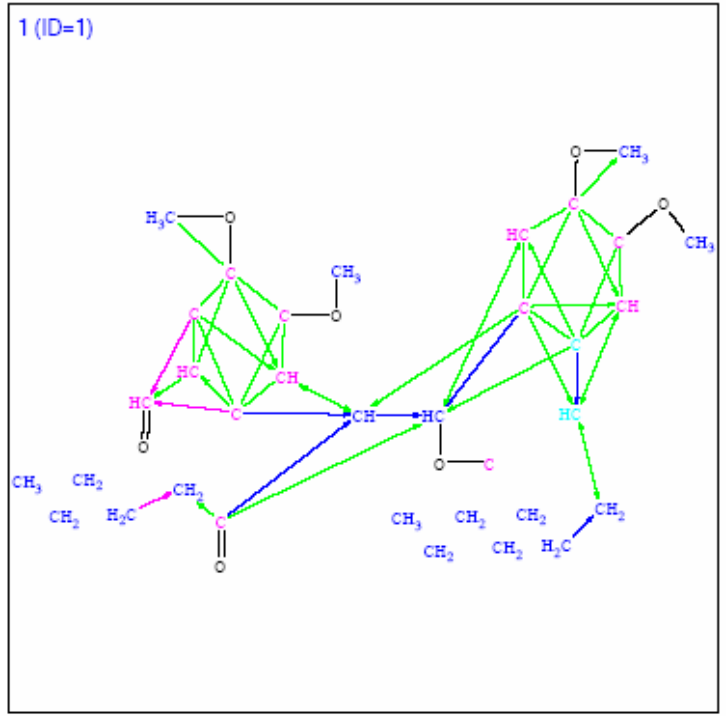




\section{Generated Structures}

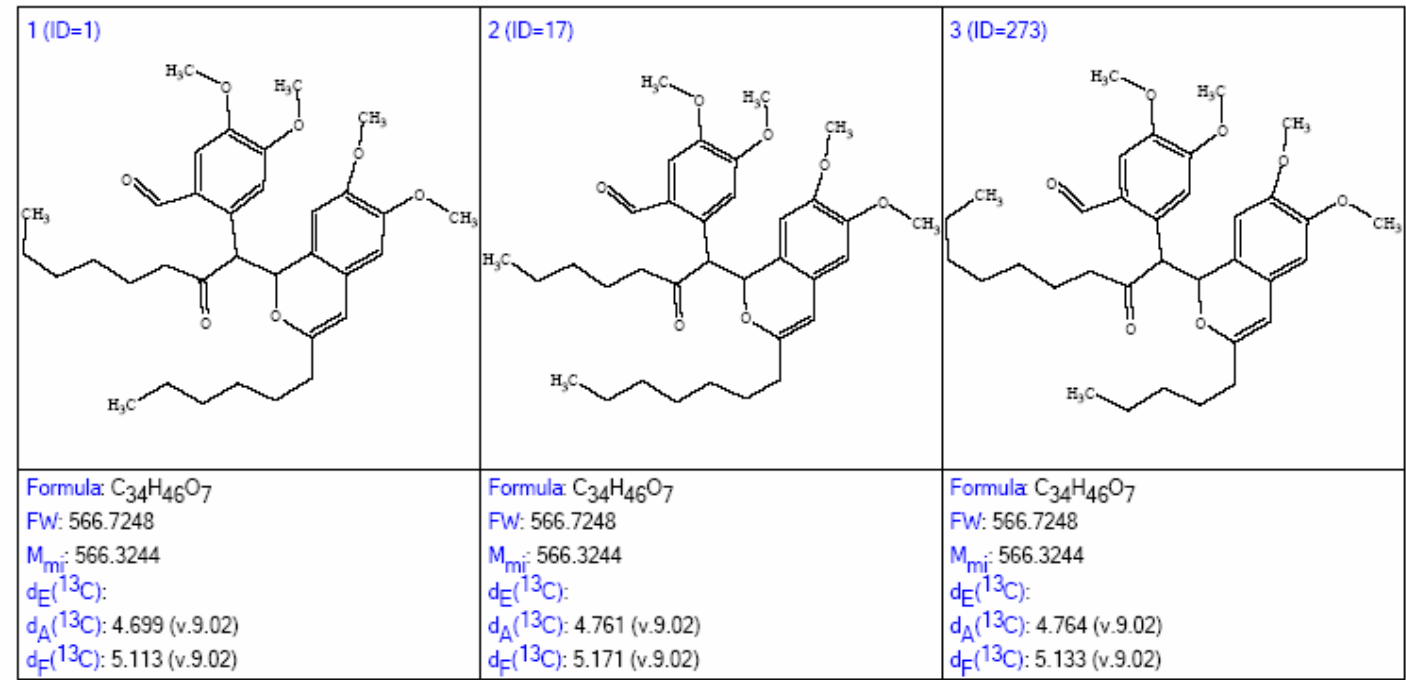




\section{ACD structure elucidation for compound 53}

${ }^{1} \mathrm{H} \mathrm{NMR}\left(400 \mathrm{MHz}, \mathrm{CDCl}_{3}\right)$
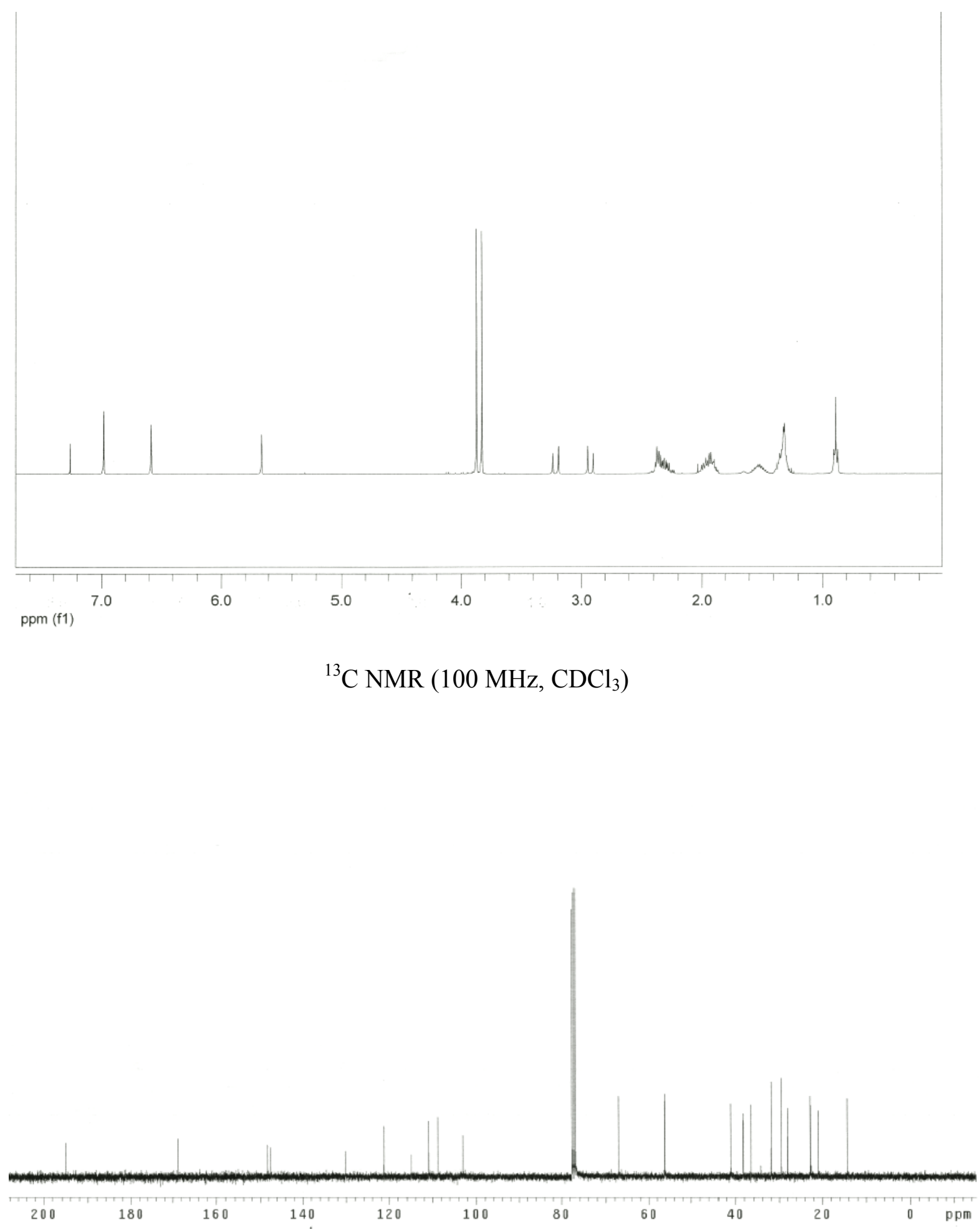


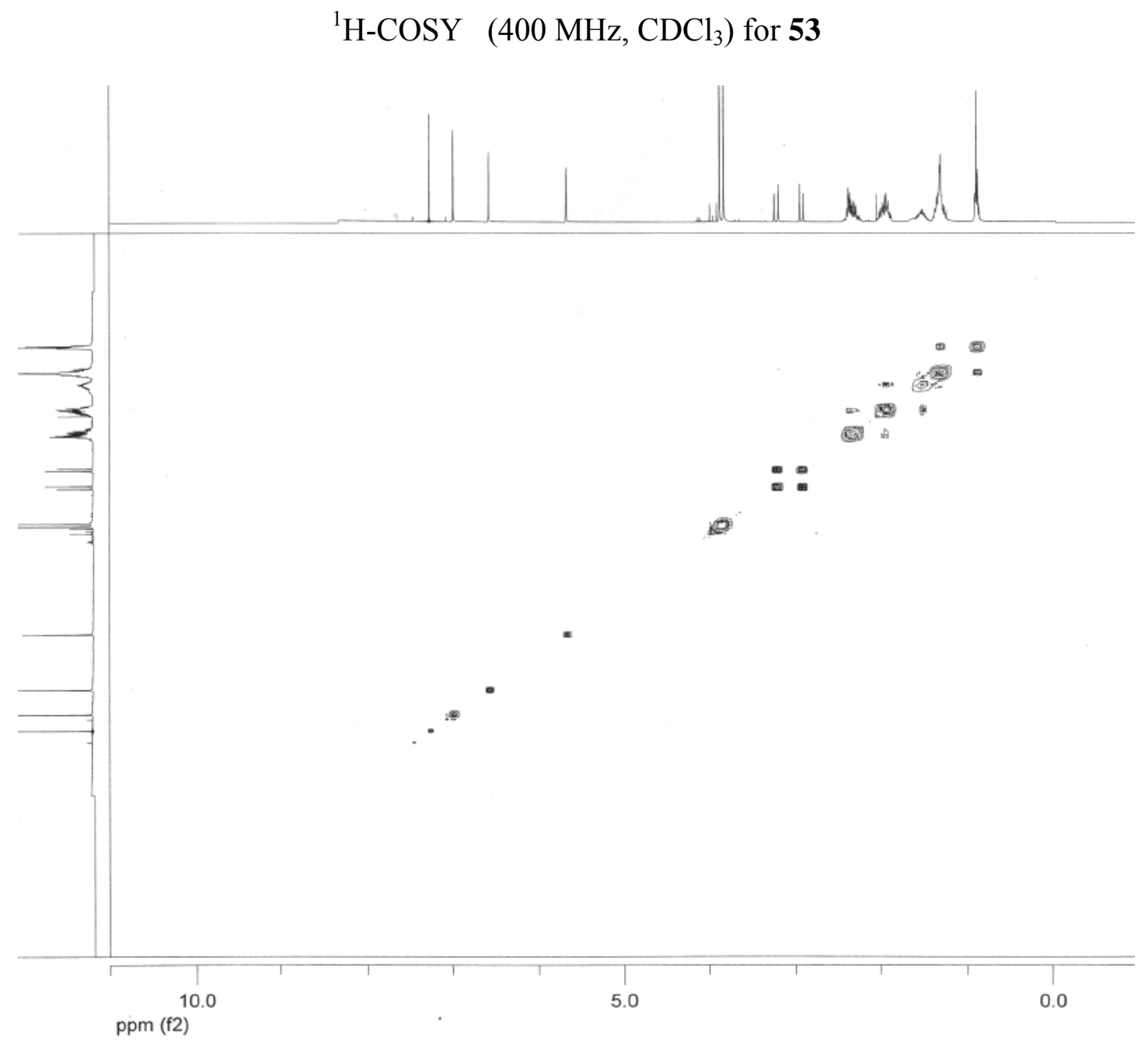


HMQC NMR (100 MHz, $\left.\mathrm{CDCl}_{3}\right)$ for 53

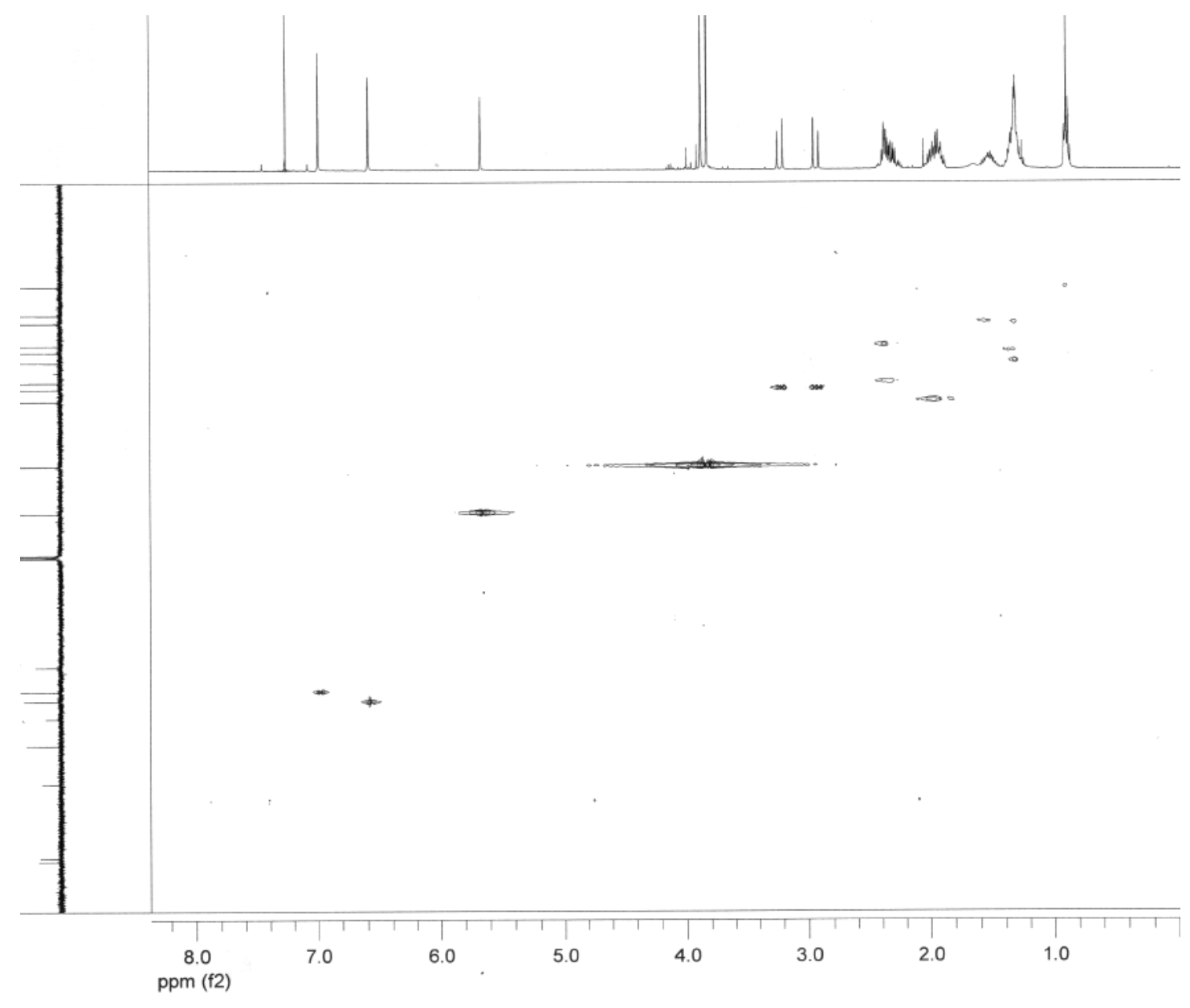


HMBC NMR (100 MHz, $\left.\mathrm{CDCl}_{3}\right)$ for 53

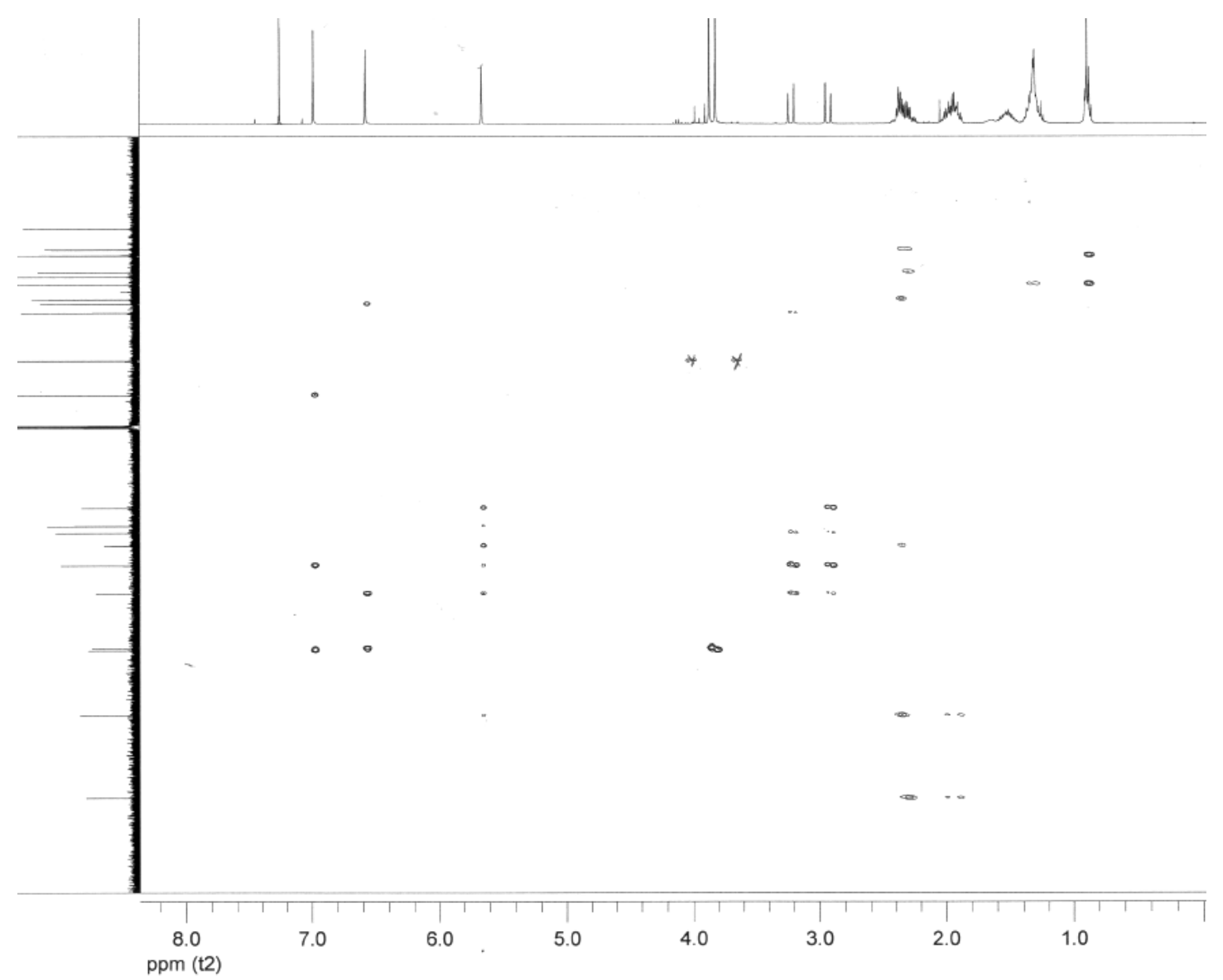


Structure Elucidator Data for 53

Initial Data

Composition Restrictions:

Molecular Weight $=386.209320$

Double Bonds Equivalent $=9$

Allowed Composition $=\mathrm{C}(23) \mathrm{H}(30) \mathrm{O}(5)$

Molecular Formula $=\mathrm{C} 23 \mathrm{H} 30 \mathrm{O} 5$

Spectral Data:

standard $13 C$ (exp) -23 peaks

COSY $1 \mathrm{H}-1 \mathrm{H}$ (exp) - 2 peaks

HMQC $13 \mathrm{C}-1 \mathrm{H}$ (exp) - 16 peaks

HMBC $13 \mathrm{C}-1 \mathrm{H}$ (exp) - 40 peaks

\section{Most Probable Structure}

Following structure was placed to the first position after spectra calculation

Carbon Assignment

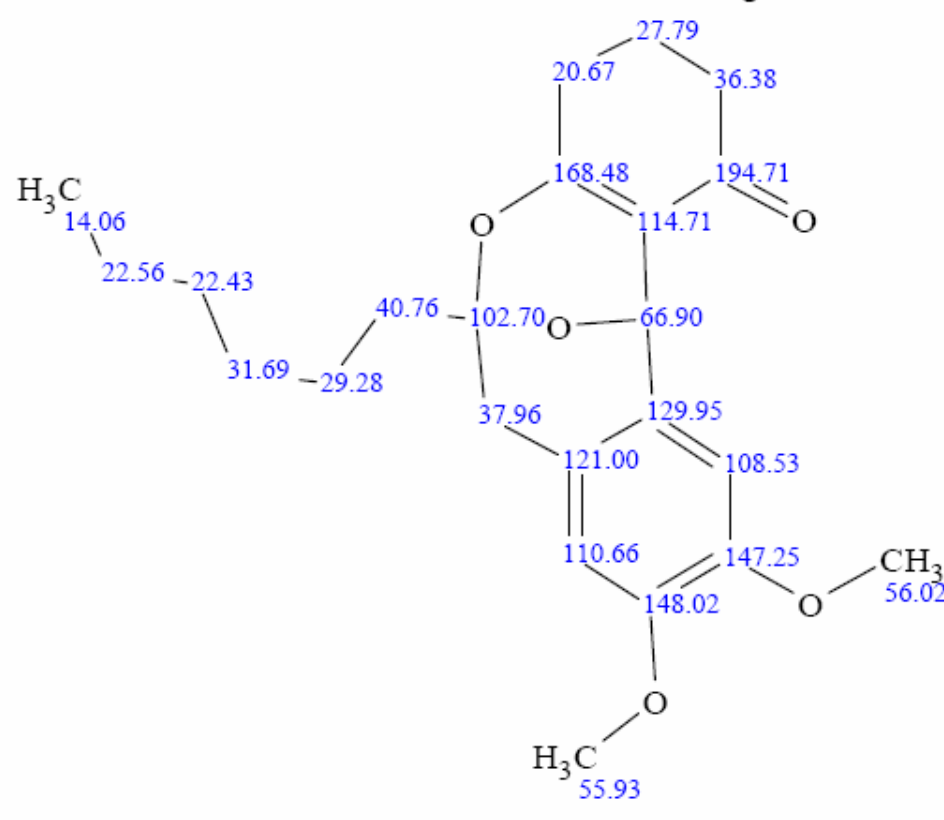

\begin{tabular}{|c|c|c|c|c|}
\hline$\#$ & $\mathrm{~N}$ & Shift (ppm) & Atoms & $\mathrm{XHn}$ \\
\hline 1 & 3 & 14.059 & 1 & $\mathrm{CH} 3(\mathrm{q})$ \\
\hline 2 & 4 & 20.675 & 1 & $\mathrm{CH} 2(\mathrm{t})$ \\
\hline 3 & 5 & 22.426 & 1 & $\mathrm{CH} 2(\mathrm{t})$ \\
\hline 4 & 6 & 22.557 & 1 & $\mathrm{CH} 2(\mathrm{t})$ \\
\hline 5 & 7 & 27.788 & 1 & $\mathrm{CH} 2(\mathrm{t})$ \\
\hline 6 & 8 & 29.276 & 1 & $\mathrm{CH} 2(\mathrm{t})$ \\
\hline 7 & 9 & 31.693 & 1 & $\mathrm{CH} 2(\mathrm{t})$ \\
\hline 8 & 10 & 36.375 & 1 & $\mathrm{CH} 2(\mathrm{t})$ \\
\hline 9 & 11 & 37.958 & 1 & $\mathrm{CH} 2(\mathrm{t})$ \\
\hline 10 & 12 & 40.756 & 1 & $\mathrm{CH} 2(\mathrm{t})$ \\
\hline 11 & 1 & 55.929 & 1 & $\mathrm{CH} 3(\mathrm{q})$ \\
\hline 12 & 2 & 56.017 & 1 & $\mathrm{CH} 3(\mathrm{q})$ \\
\hline 13 & 13 & 66.904 & 1 & $\mathrm{CH}(\mathrm{d})$ \\
\hline 14 & 20 & 102.701 & 1 & $\mathrm{C}(\mathrm{s})$ \\
\hline 15 & 14 & 108.526 & 1 & $\mathrm{CH}(\mathrm{d})$ \\
\hline 16 & 15 & 110.665 & 1 & $\mathrm{CH}(\mathrm{d})$ \\
\hline 17 & 21 & 114.709 & 1 & $\mathrm{C}(\mathrm{s})$ \\
\hline 18 & 22 & 120.995 & 1 & $\mathrm{C}(\mathrm{s})$ \\
\hline 19 & 23 & 129.948 & 1 & $\mathrm{C}(\mathrm{s})$ \\
\hline 20 & 17 & 147.246 & 1 & $\mathrm{C}(\mathrm{s})$ \\
\hline 21 & 18 & 148.022 & 1 & $\mathrm{C}(\mathrm{s})$ \\
\hline 22 & 19 & 168.478 & 1 & $\mathrm{C}(\mathrm{s})$ \\
\hline 23 & 16 & 194.706 & 1 & $\mathrm{C}(\mathrm{s})$ \\
\hline
\end{tabular}




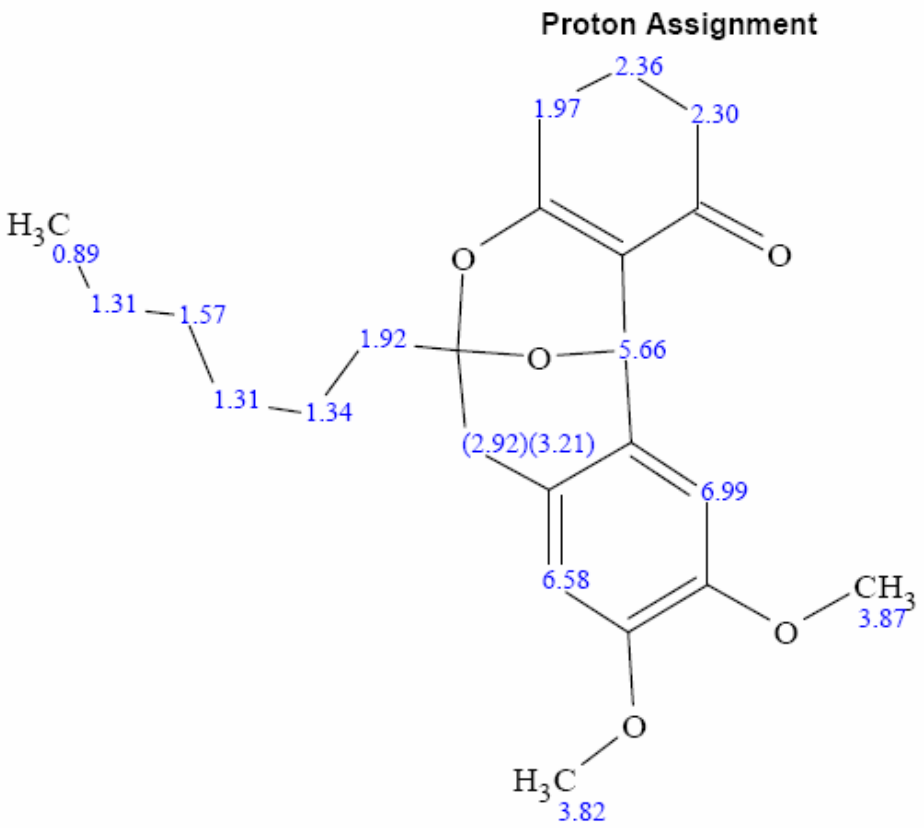

\begin{tabular}{|c|c|c|c|}
\hline$\#$ & $\mathrm{~N}$ & Shift (ppm) & Protons \\
\hline 1 & 3 & 0.889 & 3 \\
\hline 2 & 6 & 1.310 & 2 \\
\hline 3 & 9 & 1.310 & 2 \\
\hline 4 & 8 & 1.341 & 2 \\
\hline 5 & 5 & 1.567 & 2 \\
\hline 6 & 12 & 1.919 & 2 \\
\hline 7 & 4 & 1.973 & 2 \\
\hline 8 & 10 & 2.304 & 2 \\
\hline 9 & 7 & 2.363 & 2 \\
\hline 10 & 11 & 2.925 & 1 \\
\hline 11 & 11 & 3.213 & 1 \\
\hline 12 & 1 & 3.823 & 3 \\
\hline 13 & 2 & 3.870 & 3 \\
\hline 14 & 13 & 5.660 & 1 \\
\hline 15 & 15 & 6.580 & 1 \\
\hline 16 & 14 & 6.985 & 1 \\
\hline
\end{tabular}




\section{Molecular Connectivity Diagrams (MCDs)}

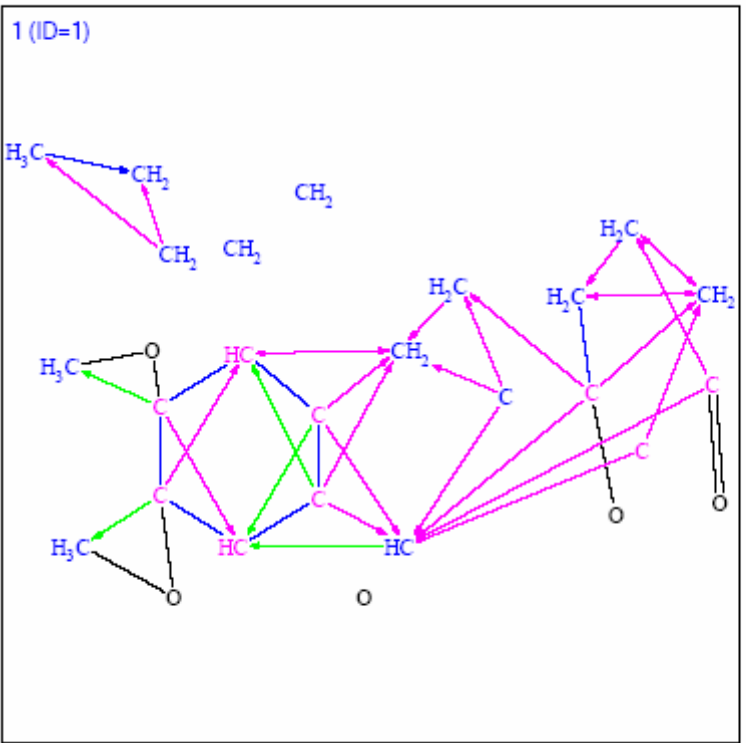

\section{Generated Structures}

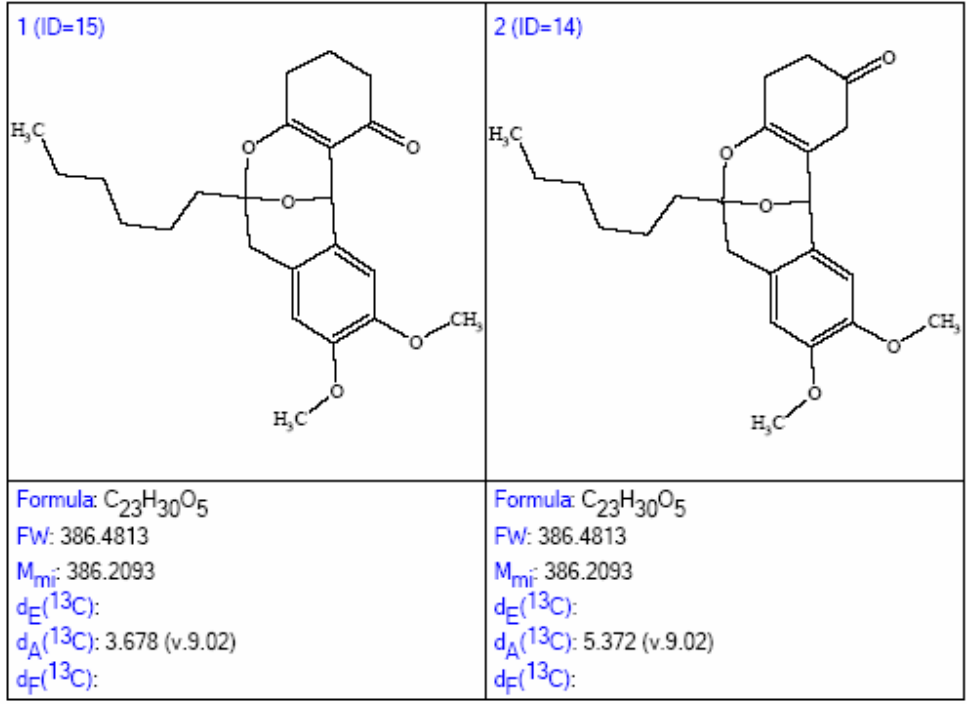




\section{Select NMR Spectra}

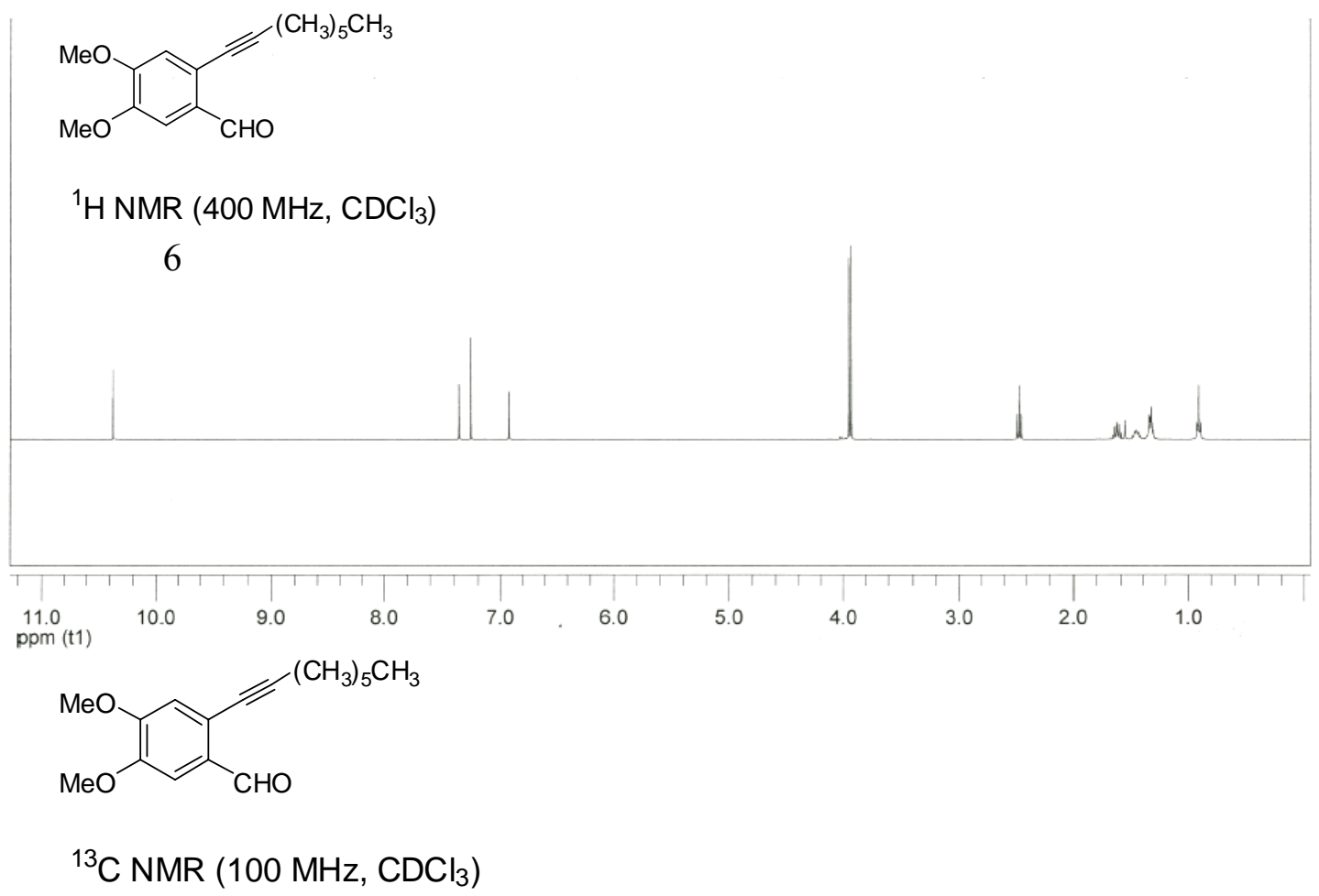

6

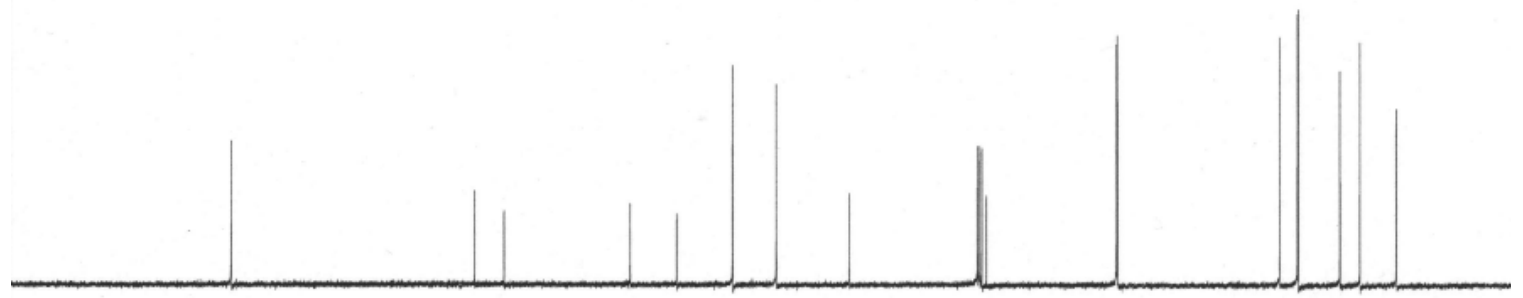

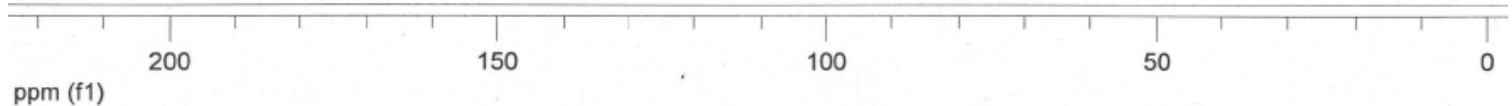




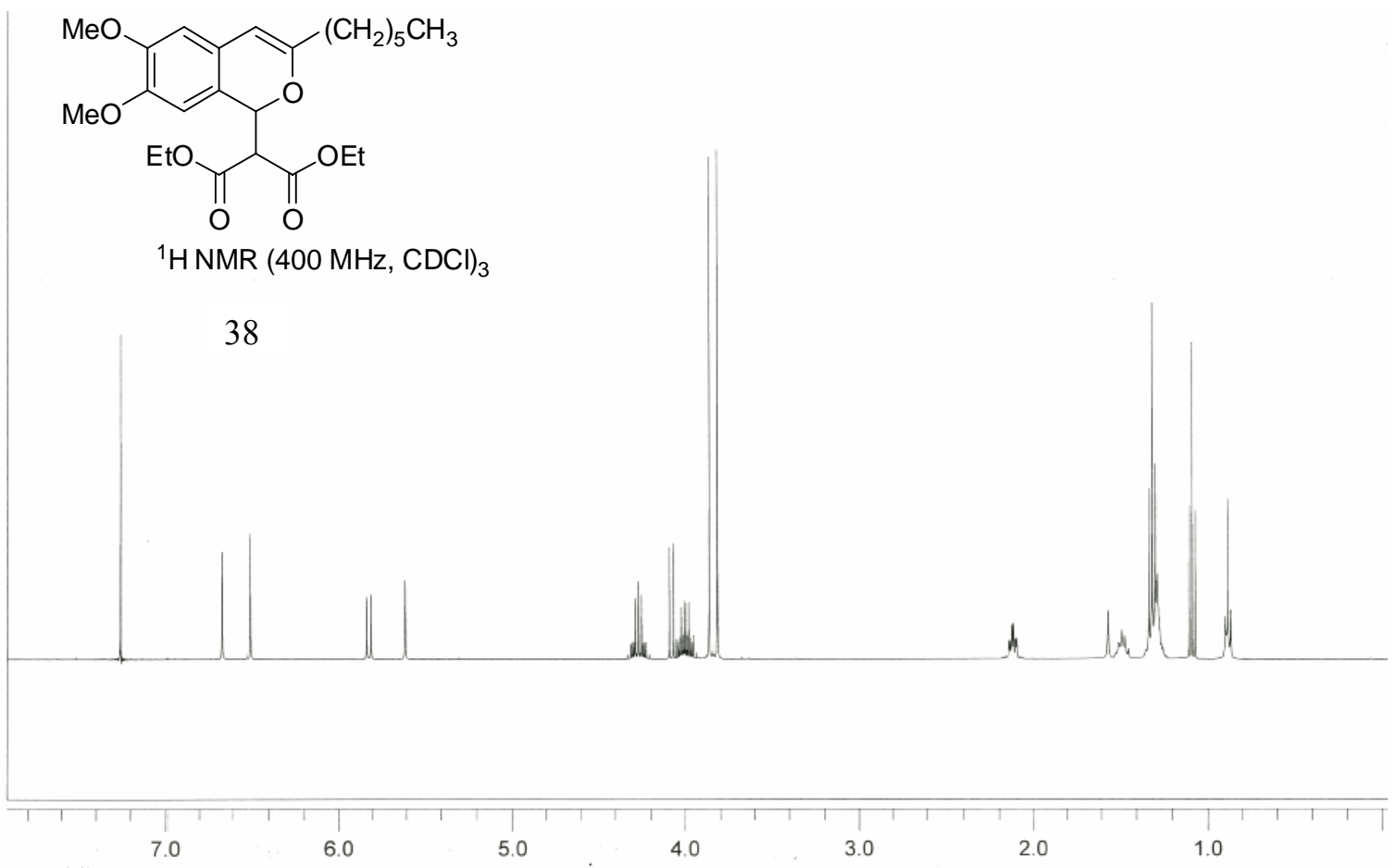

ppm (t1)

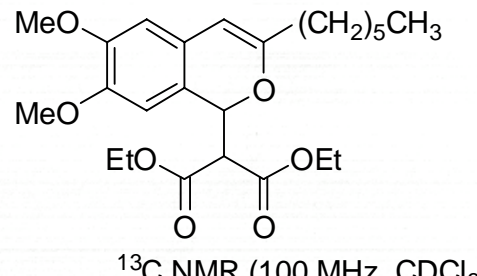

38

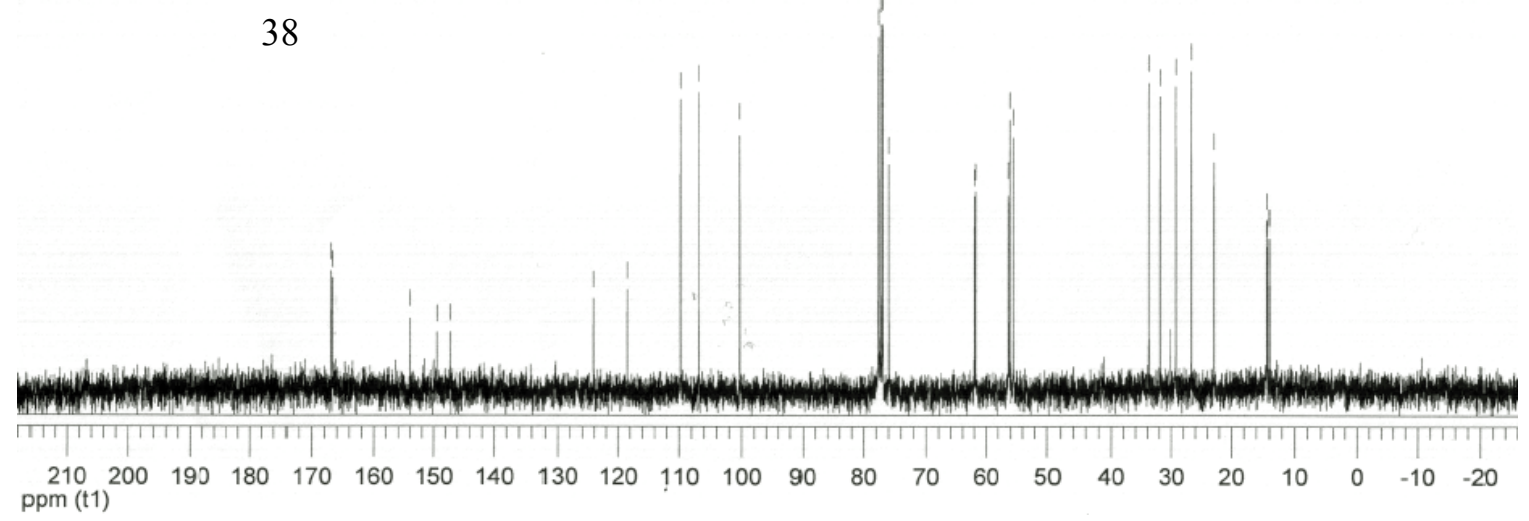



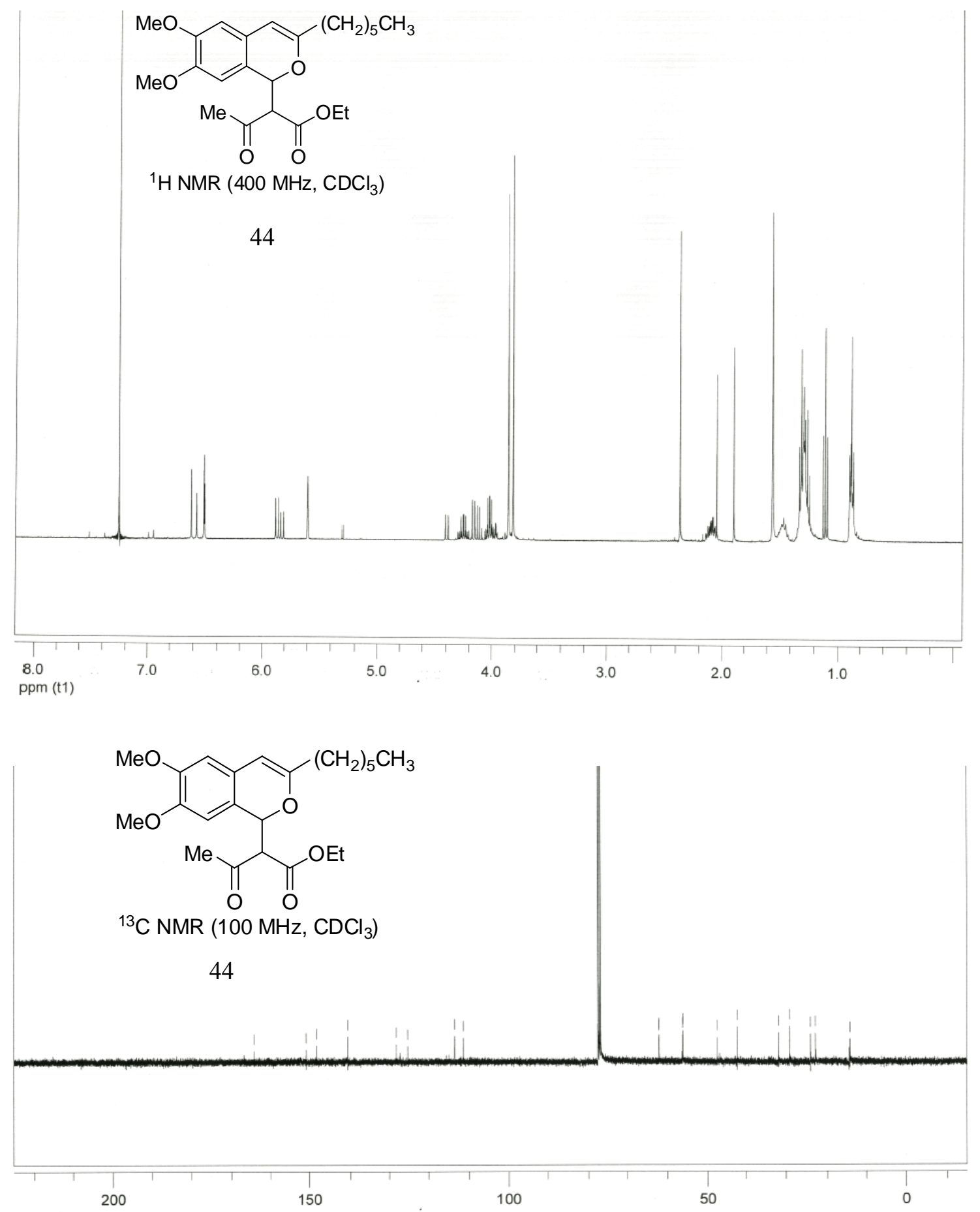

ppm (f1) 

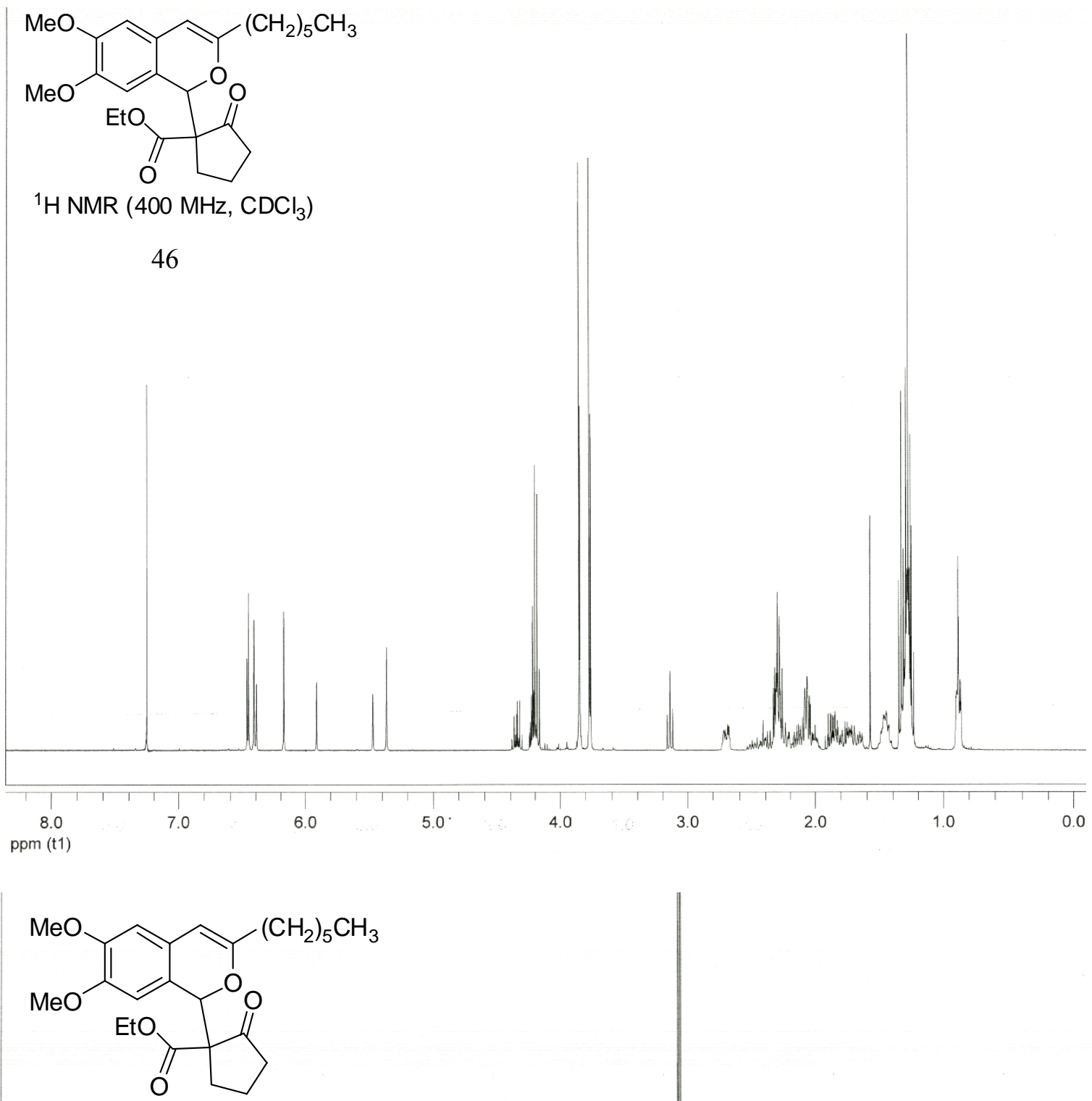

${ }^{13} \mathrm{C}$ NMR $\left(100 \mathrm{MHz}, \mathrm{CDCl}_{3}\right)$

46
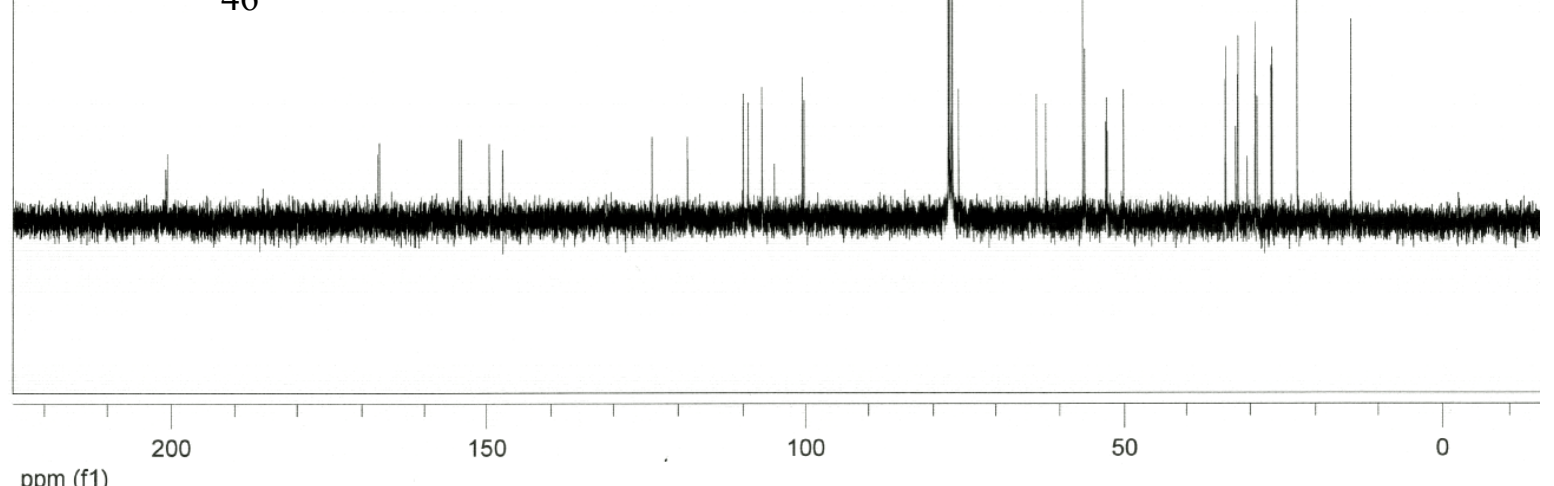


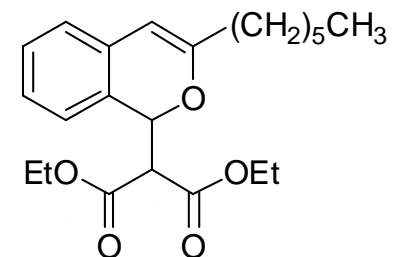

${ }^{1} \mathrm{H}$ NMR $\left(400 \mathrm{MHz}, \mathrm{CDCl}_{3}\right)$
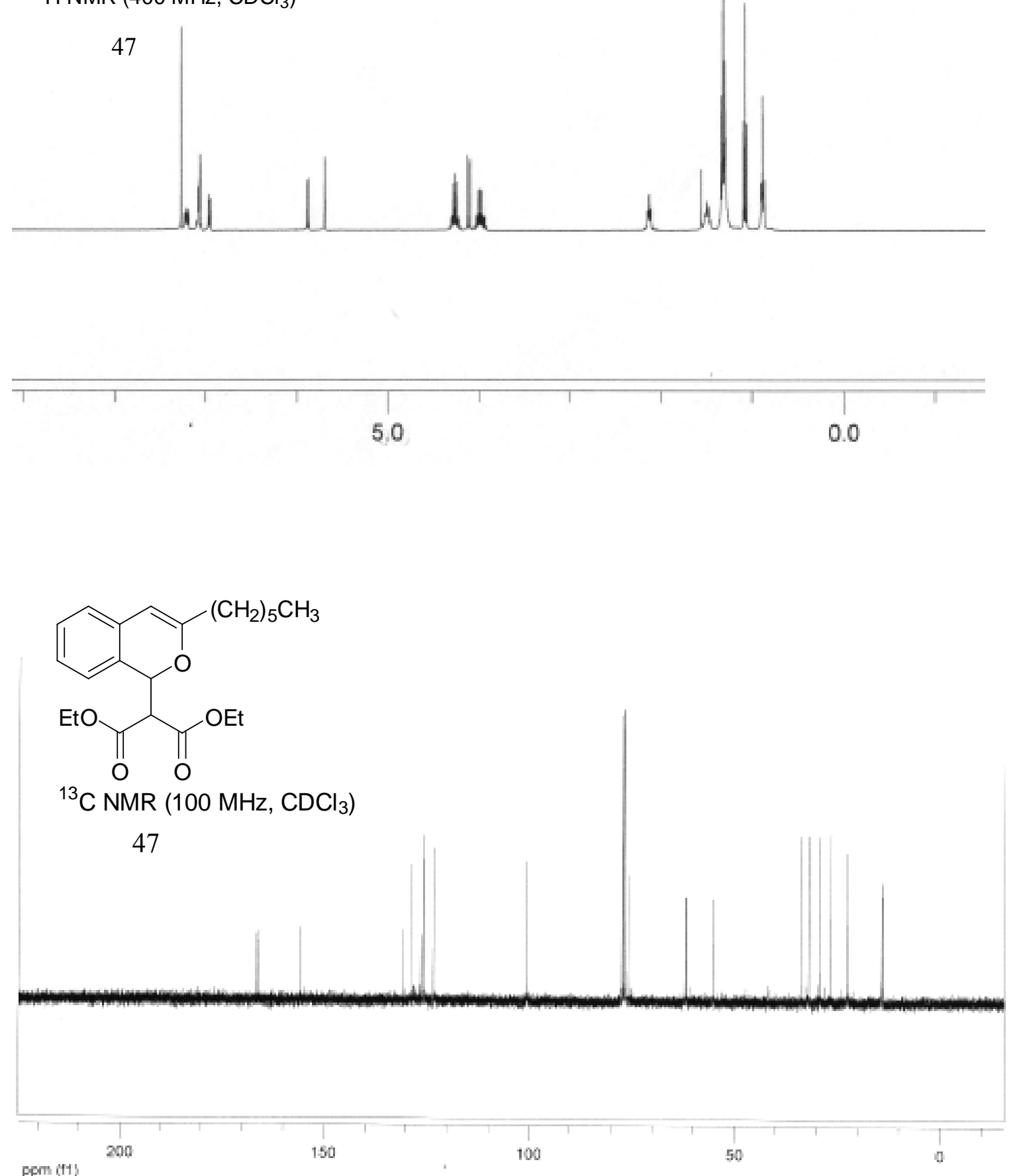


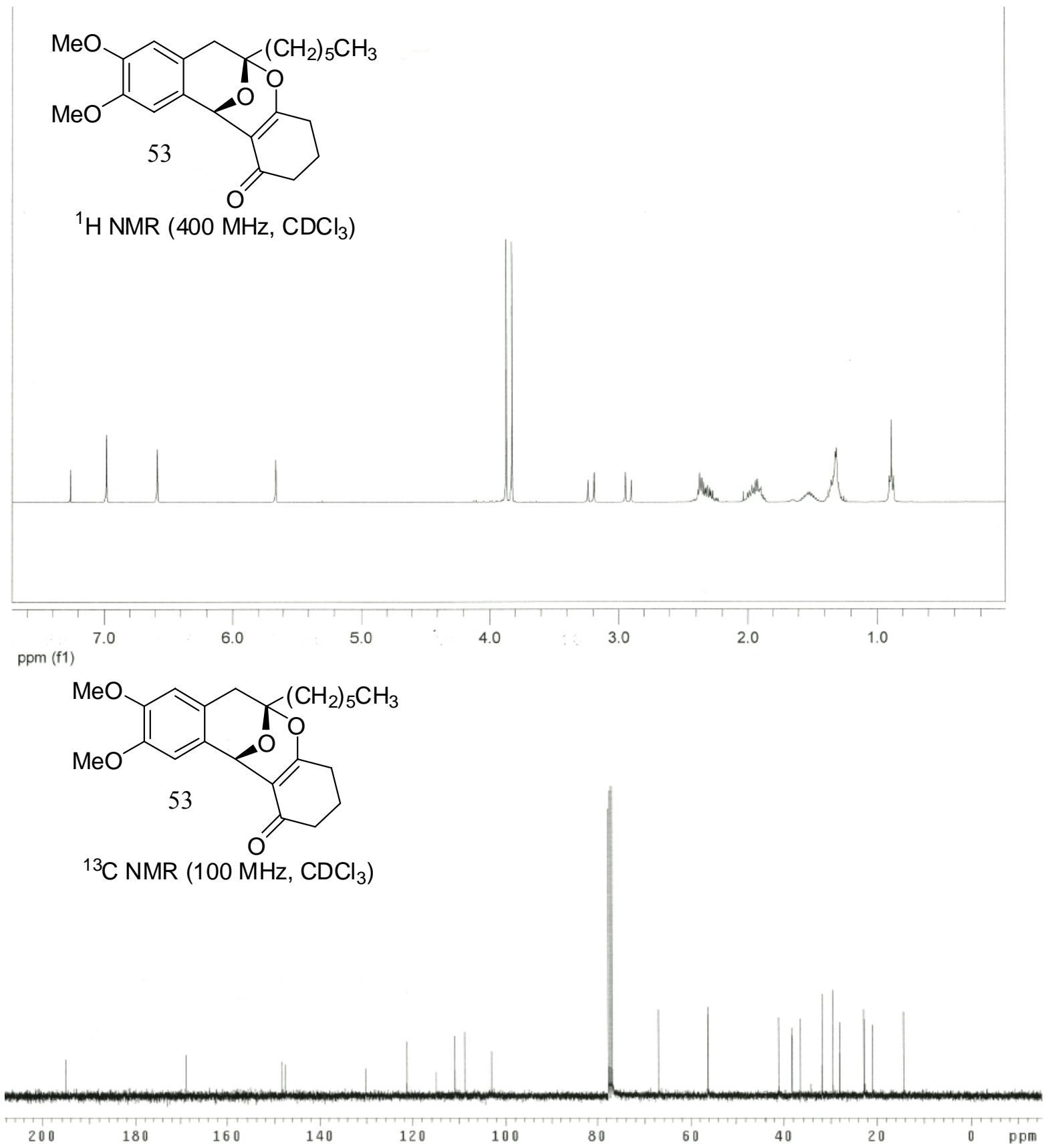




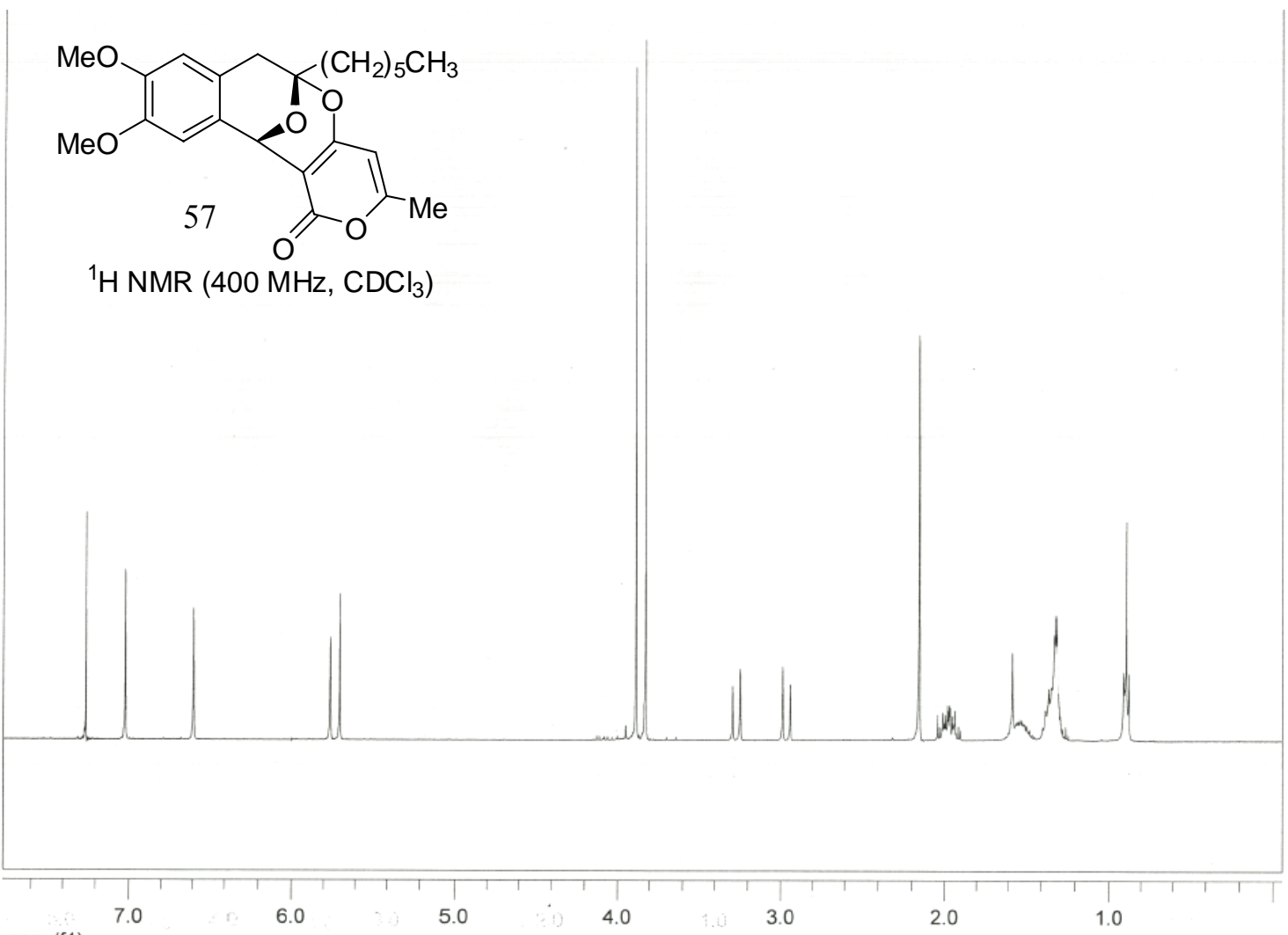

ppm (f1)

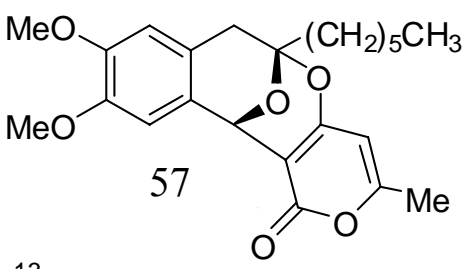

${ }^{13} \mathrm{C}$ NMR $\left(100 \mathrm{MHz}, \mathrm{CDCl}_{3}\right)$
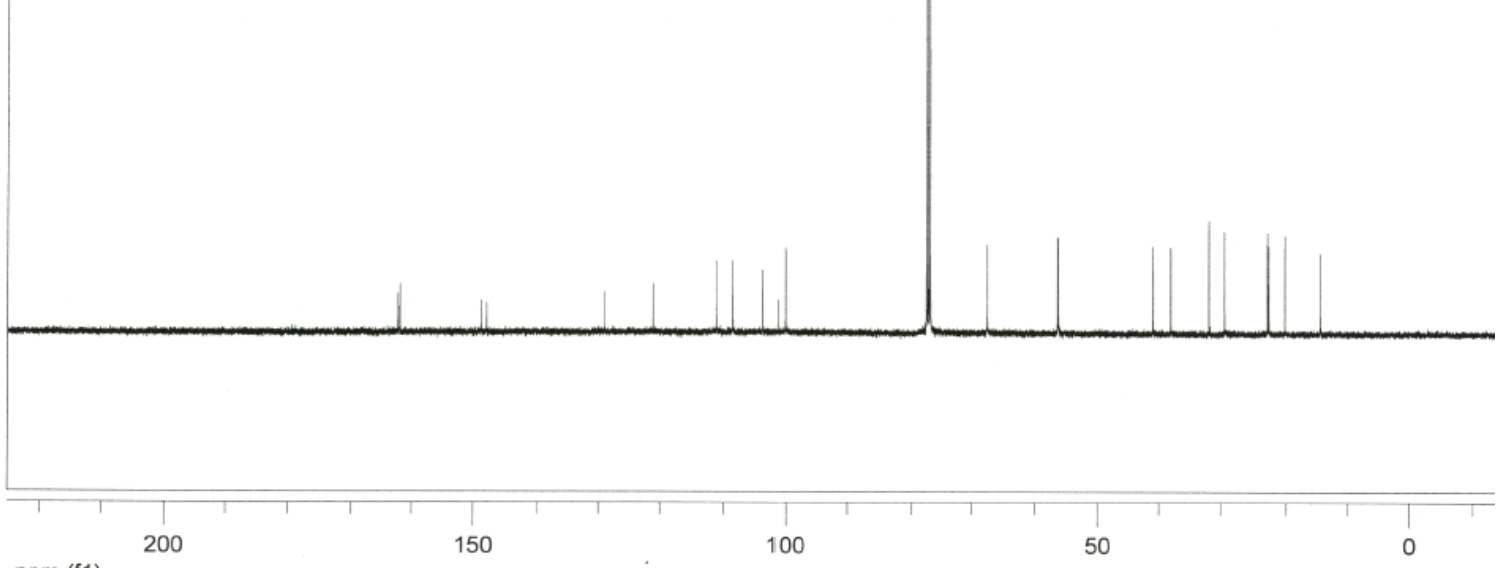

ppm (f1) 


\section{VIII. ${ }^{1} \mathrm{H}$-NOESY (400 MHz, $\mathrm{CDCl}_{3}$ ) analysis of 39 and 39'}
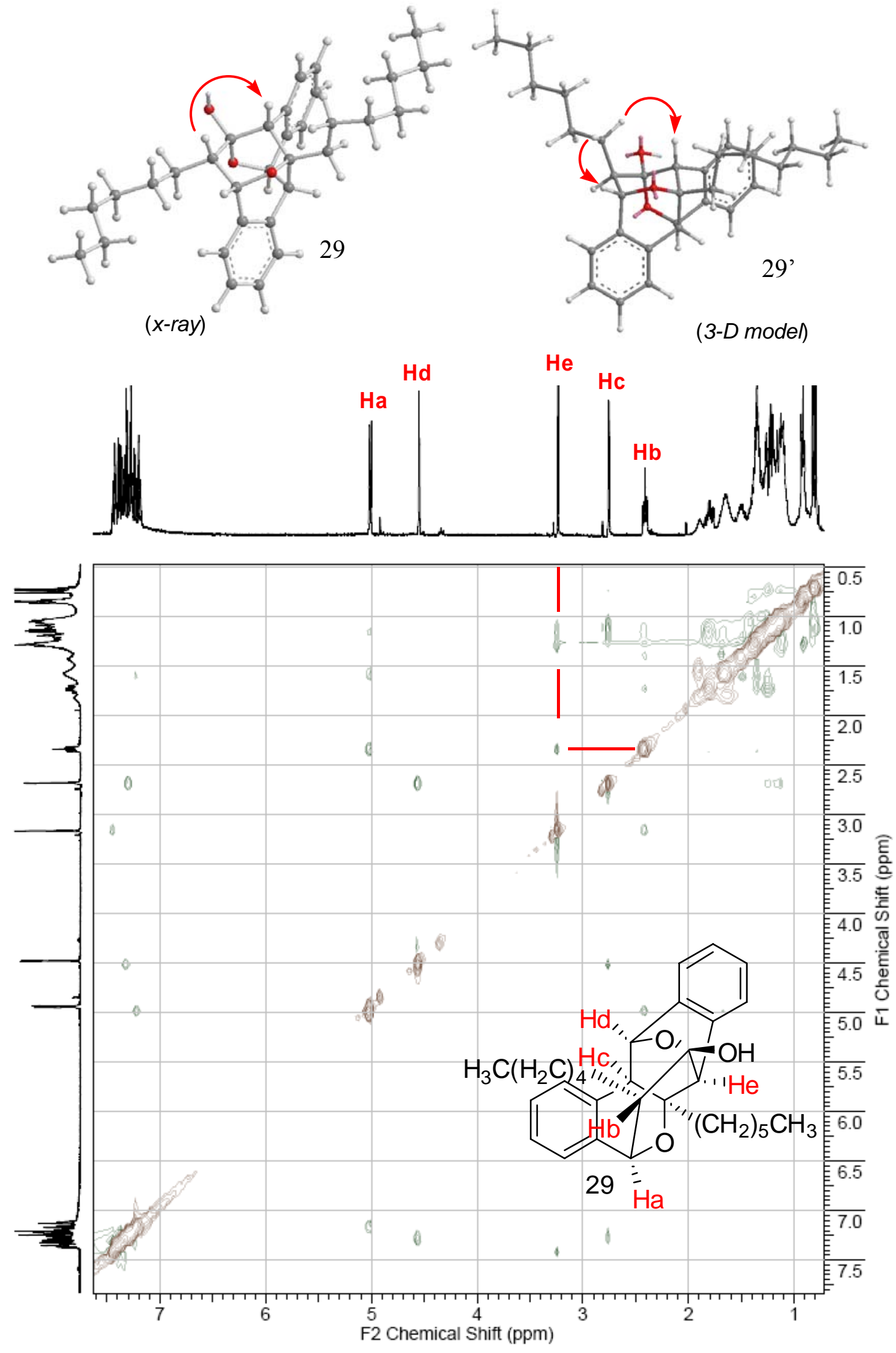


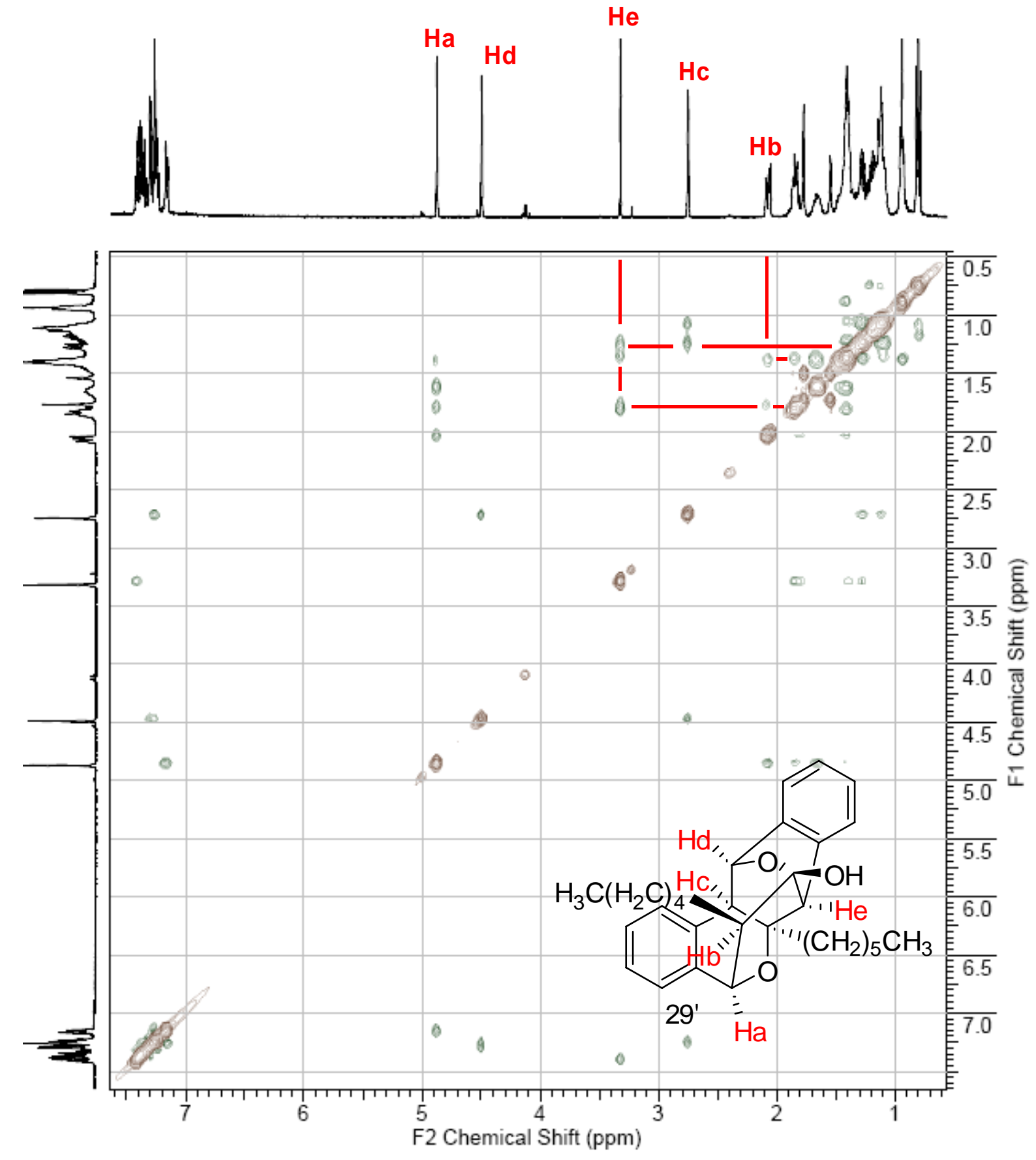




\section{NMR Analysis of $\mathbf{6 6}$}

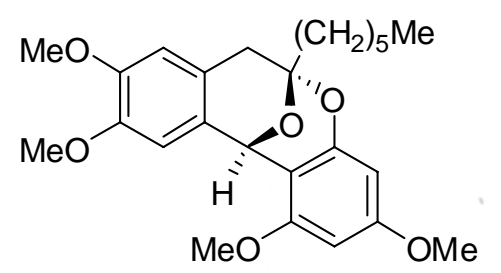

${ }^{1} \mathrm{H}$ NMR, (400 MHz, $\mathrm{CDCl}_{3}$ )

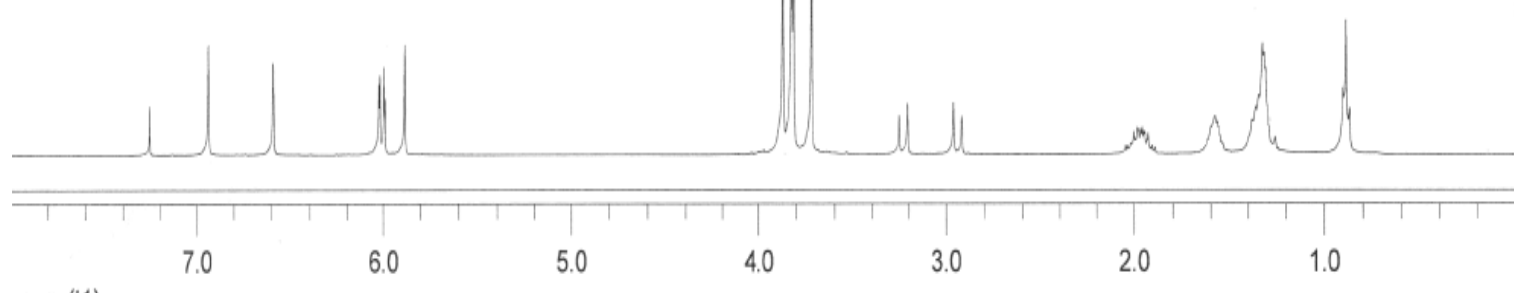

ppm (t1)

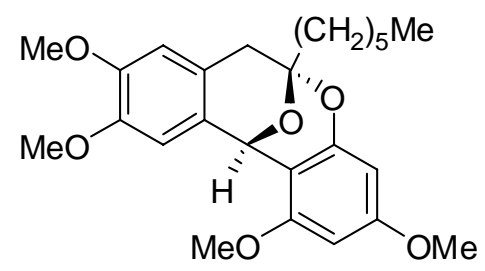

${ }^{13} \mathrm{C}$ NMR (100 MHz, $\left.\mathrm{CDCl}_{3}\right)$

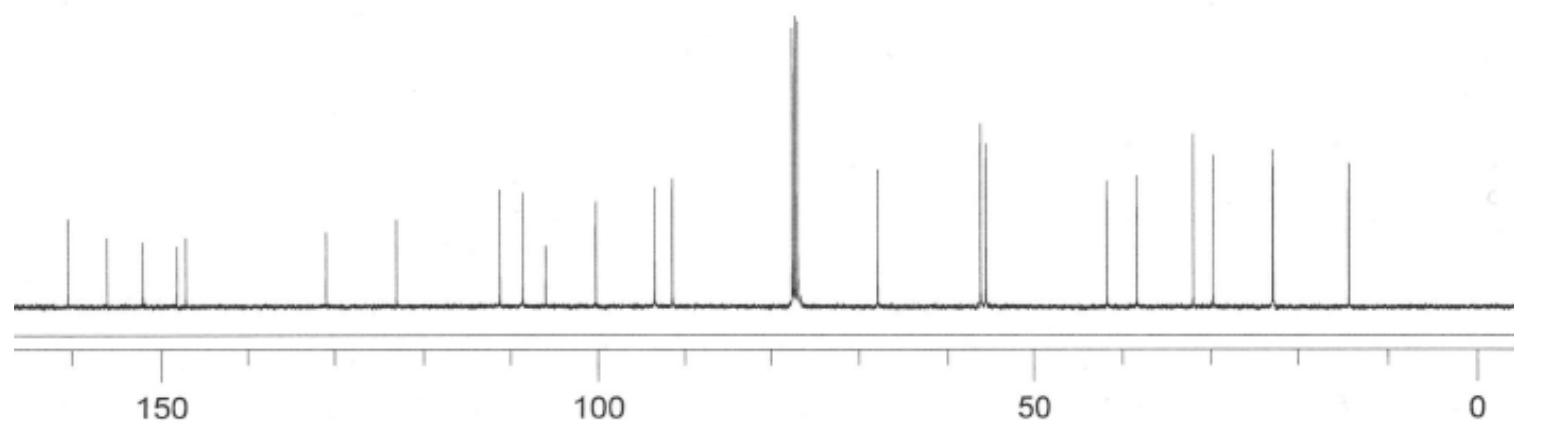




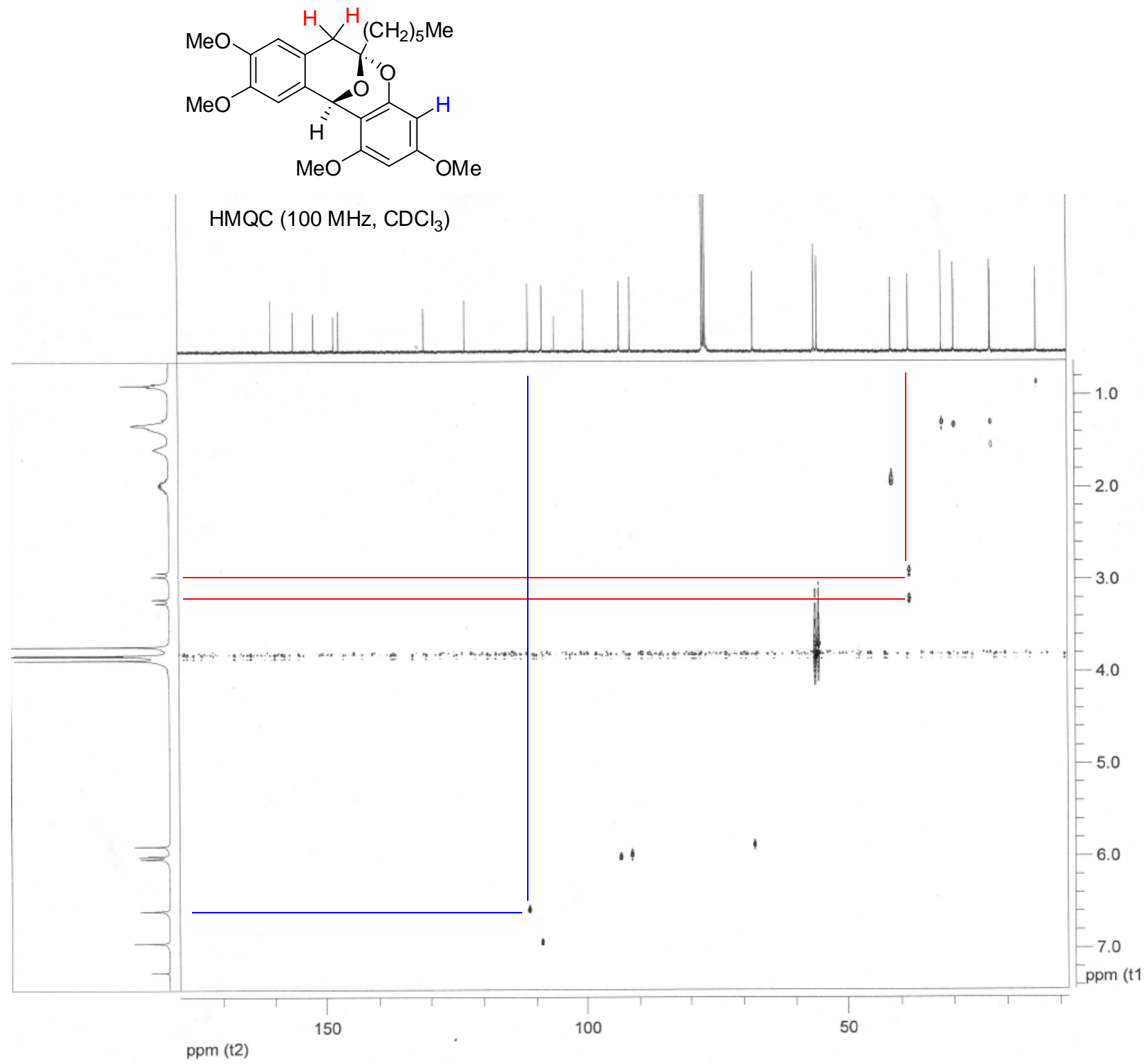



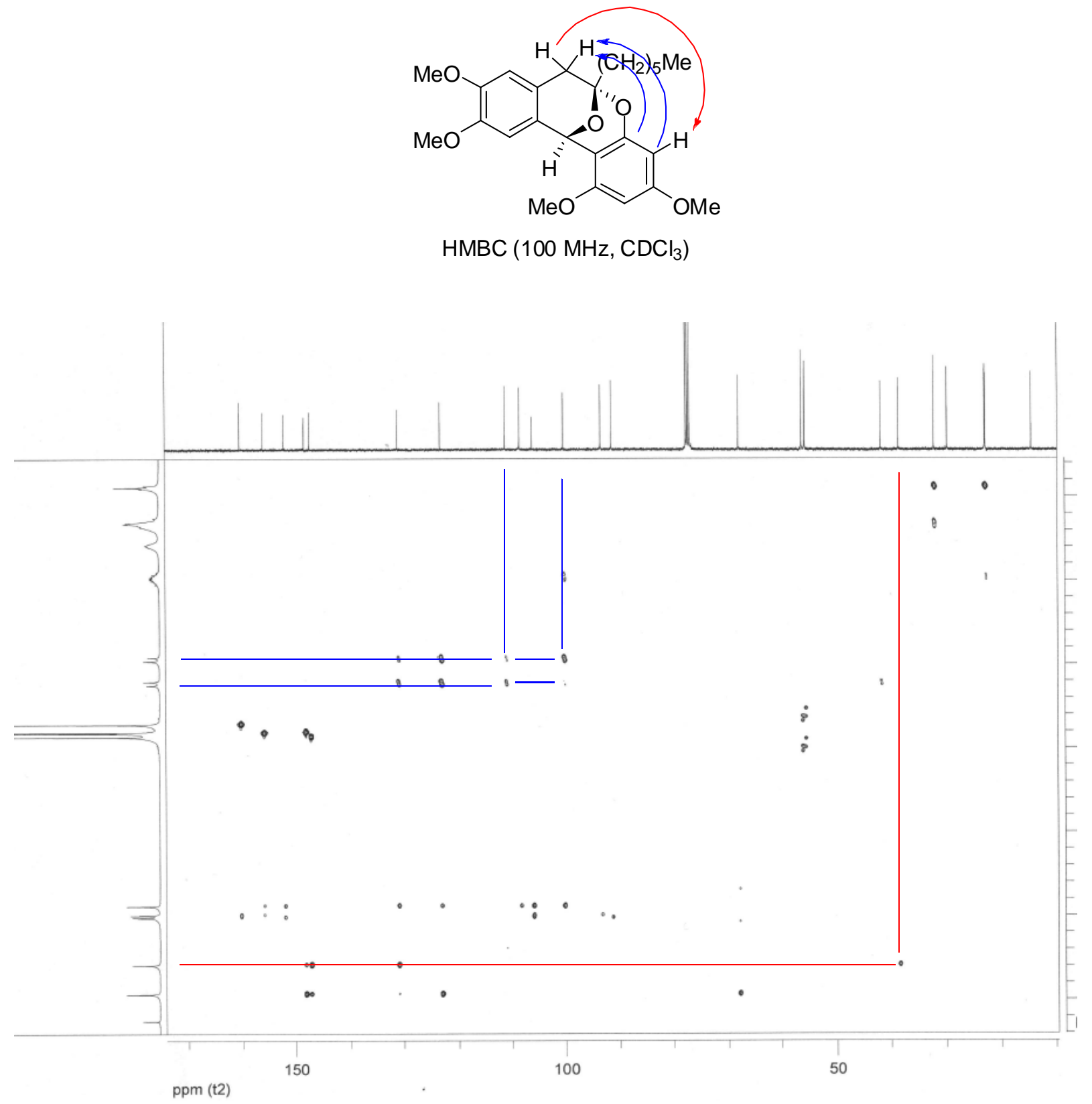


\section{X-ray Crystal Structure Data}

\section{X-ray Crystal Structure of Compound 29:}

Crystals of compound 29 suitable for x-ray analysis were obtained by slow evaporation from hexane. Crystallographic data have been deposited with the Cambridge Crystallographic Data Centre (CCDC \# 619278). Copies of the data can be obtained free of charge onapplication to the CCDC, 12 Union Road, Cambridge CB21EZ, UK (fax: (+44)-1223-336-033;e-mail: deposit@ccdc.cam.ac.uk.

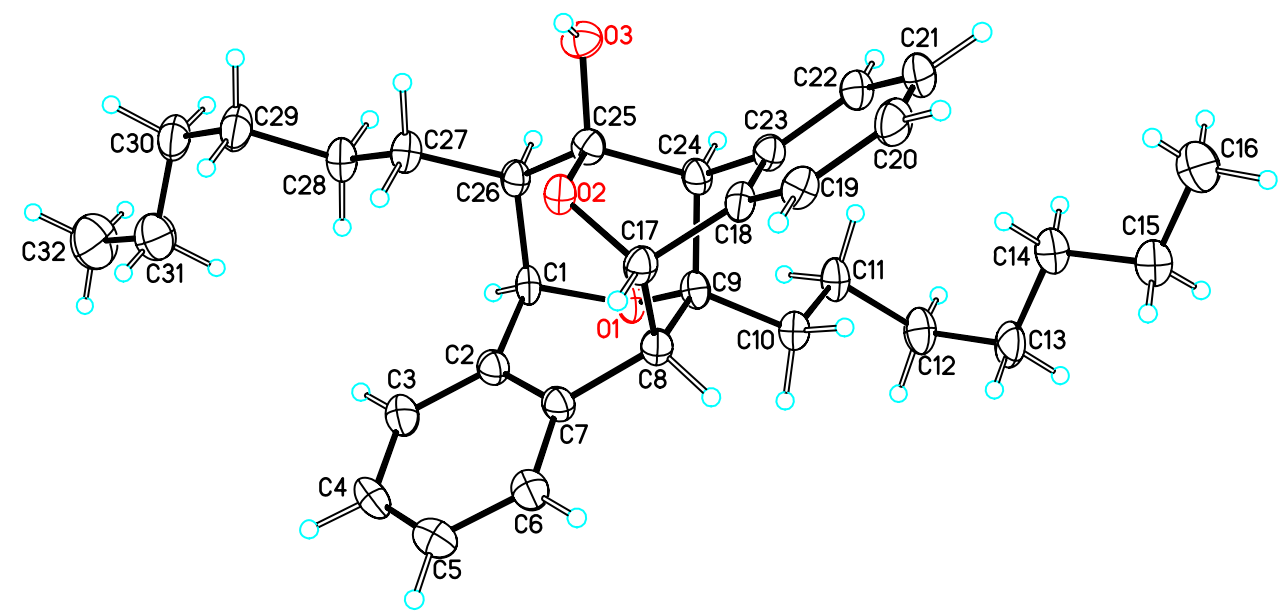

Table 1. Crystal data and structure refinement for 29.

Identification code

Empirical formula

Formula weight

Temperature

Wavelength

Crystal system

Space group

Unit cell dimensions

Volume

Z

Density (calculated)

\section{9}

C32 H42 O3

474.66

173(2) K

$0.71073 \AA$

Orthorhombic

Pbca

$$
\begin{array}{ll}
\mathrm{a}=18.309(4) \AA & \alpha=90^{\circ} . \\
\mathrm{b}=10.249(2) \AA & \beta=90^{\circ} . \\
\mathrm{c}=28.639(5) \AA & \gamma=90^{\circ} .
\end{array}
$$$$
\text { 5373.8(17) } \AA^{3}
$$

8

$1.173 \mathrm{Mg} / \mathrm{m}^{3}$ 


$\begin{array}{ll}\text { Absorption coefficient } & 0.073 \mathrm{~mm}^{-1} \\ \mathrm{~F}(000) & 2064 \\ \text { Crystal size } & 0.50 \times 0.10 \times 0.03 \mathrm{~mm}^{3} \\ \text { Theta range for data collection } & 2.22 \text { to } 20.91^{\circ} . \\ \text { Index ranges } & -18<=\mathrm{h}<=18,-10<=\mathrm{k}<=10,-28<=\mathrm{k}<=28 \\ \text { Reflections collected } & 45099 \\ \text { Independent reflections } & 2834[\mathrm{R}(\mathrm{int})=0.1668] \\ \text { Completeness to theta }=20.91^{\circ} & 99.3 \% \\ \text { Absorption correction } & \mathrm{Semi}-\mathrm{empirical} \mathrm{from} \mathrm{equivalents} \\ \text { Max. and min. transmission } & 0.9982 \text { and } 0.9643 \\ \text { Refinement method } & \text { Full-matrix least-squares on } \mathrm{F}^{2} \\ \text { Data / restraints / parameters } & 2834 / 0 / 317 \\ \text { Goodness-of-fit on } \mathrm{F}^{2} & 1.059 \\ \text { Final R indices [I }>2 \text { sigma(I)] } & \mathrm{R} 1=0.0466, \text { wR2 }=0.1079 \\ \text { R indices (all data) } & \mathrm{R} 1=0.0811, \text { wR2 }=0.1317 \\ \text { Extinction coefficient } & 0.0076(9) \\ \text { Largest diff. peak and hole } & 0.211 \text { and }-0.194 \text { e. } \AA^{-3}\end{array}$

Table 2. Atomic coordinates $\left(\times 10^{4}\right)$ and equivalent isotropic displacement parameters $\left(\AA^{2} \times 10^{3}\right)$ for $29 \mathrm{U}(\mathrm{eq})$ is defined as one third of the trace of the orthogonalized $U^{\mathrm{ij}}$ tensor.

\begin{tabular}{lcccc}
\hline & $\mathrm{x}$ & $\mathrm{y}$ & $\mathrm{z}$ & $\mathrm{U}(\mathrm{eq})$ \\
\hline $\mathrm{O}(1)$ & & & \\
$\mathrm{O}(2)$ & $1317(1)$ & $-2669(2)$ & $6873(1)$ & $28(1)$ \\
$\mathrm{O}(3)$ & $1937(1)$ & $509(2)$ & $7067(1)$ & $28(1)$ \\
$\mathrm{C}(1)$ & $3044(1)$ & $-164(2)$ & $6760(1)$ & $33(1)$ \\
$\mathrm{C}(2)$ & $1645(2)$ & $-2311(3)$ & $7318(1)$ & $28(1)$ \\
$\mathrm{C}(3)$ & $1069(2)$ & $-1582(3)$ & $7587(1)$ & $28(1)$ \\
$\mathrm{C}(4)$ & $940(2)$ & $-1804(3)$ & $8062(1)$ & $38(1)$ \\
$\mathrm{C}(5)$ & $378(2)$ & $-1180(4)$ & $8289(1)$ & $44(1)$ \\
$\mathrm{C}(6)$ & $-76(2)$ & $-340(4)$ & $8051(1)$ & $45(1)$ \\
$\mathrm{C}(7)$ & $43(2)$ & $-123(3)$ & $7578(1)$ & $36(1)$ \\
$\mathrm{C}(8)$ & $611(2)$ & $-736(3)$ & $7345(1)$ & $29(1)$ \\
$\mathrm{C}(9)$ & $778(2)$ & $-431(3)$ & $6842(1)$ & $27(1)$ \\
$\mathrm{C}(10)$ & $1184(2)$ & $-1551(3)$ & $6576(1)$ & $26(1)$ \\
$\mathrm{C}(11)$ & $722(2)$ & $-2084(3)$ & $6177(1)$ & $30(1)$ \\
& $1083(2)$ & $-3139(3)$ & $5882(1)$ & $34(1)$
\end{tabular}




$\begin{array}{lrrrr}\mathrm{C}(12) & 560(2) & -3798(3) & 5536(1) & 42(1) \\ \mathrm{C}(13) & 206(2) & -2885(3) & 5182(1) & 43(1) \\ \mathrm{C}(14) & 738(2) & -2104(3) & 4893(1) & 44(1) \\ \mathrm{C}(15) & 381(2) & -1318(4) & 4506(1) & 54(1) \\ \mathrm{C}(16) & 900(2) & -511(4) & 4228(1) & 70(1) \\ \mathrm{C}(17) & 1263(2) & 799(3) & 6820(1) & 28(1) \\ \mathrm{C}(18) & 1458(2) & 1127(3) & 6324(1) & 27(1) \\ \mathrm{C}(19) & 1321(2) & 2286(3) & 6094(1) & 32(1) \\ \mathrm{C}(20) & 1551(2) & 2420(3) & 5632(1) & 39(1) \\ \mathrm{C}(21) & 1916(2) & 1416(4) & 5414(1) & 39(1) \\ \mathrm{C}(22) & 2068(2) & 262(3) & 5646(1) & 32(1) \\ \mathrm{C}(23) & 1833(2) & 125(3) & 6105(1) & 28(1) \\ \mathrm{C}(24) & 1937(2) & -1033(3) & 6423(1) & 27(1) \\ \mathrm{C}(25) & 2328(2) & -570(3) & 6871(1) & 26(1) \\ \mathrm{C}(26) & 2381(2) & -1653(3) & 7231(1) & 26(1) \\ \mathrm{C}(27) & 2752(2) & -1205(3) & 7686(1) & 31(1) \\ \mathrm{C}(28) & 2976(2) & -2332(3) & 8003(1) & 37(1) \\ \mathrm{C}(29) & 3383(2) & -1870(4) & 8435(1) & 44(1) \\ \mathrm{C}(30) & 3597(2) & -2966(3) & 8769(1) & 45(1) \\ \mathrm{C}(31) & 2973(2) & -3599(4) & 9014(1) & 64(1) \\ \mathrm{C}(32) & 3205(2) & -4647(5) & 9358(1) & 82(2)\end{array}$




\section{X-ray Crystal Structure of Compound 57:}

Crystals of compound $\mathbf{5 7}$ suitable for x-ray analysis were obtained by slow evaporation from hexane. Crystallographic data have been deposited with the Cambridge Crystallographic Data Centre (CCDC \# 619279). Copies of the data can be obtained free of charge on application to the CCDC, 12 Union Road, Cambridge CB21EZ, UK (fax: (+44)-1223-336-033; ;-mail: deposit@ccdc.cam.ac.uk.

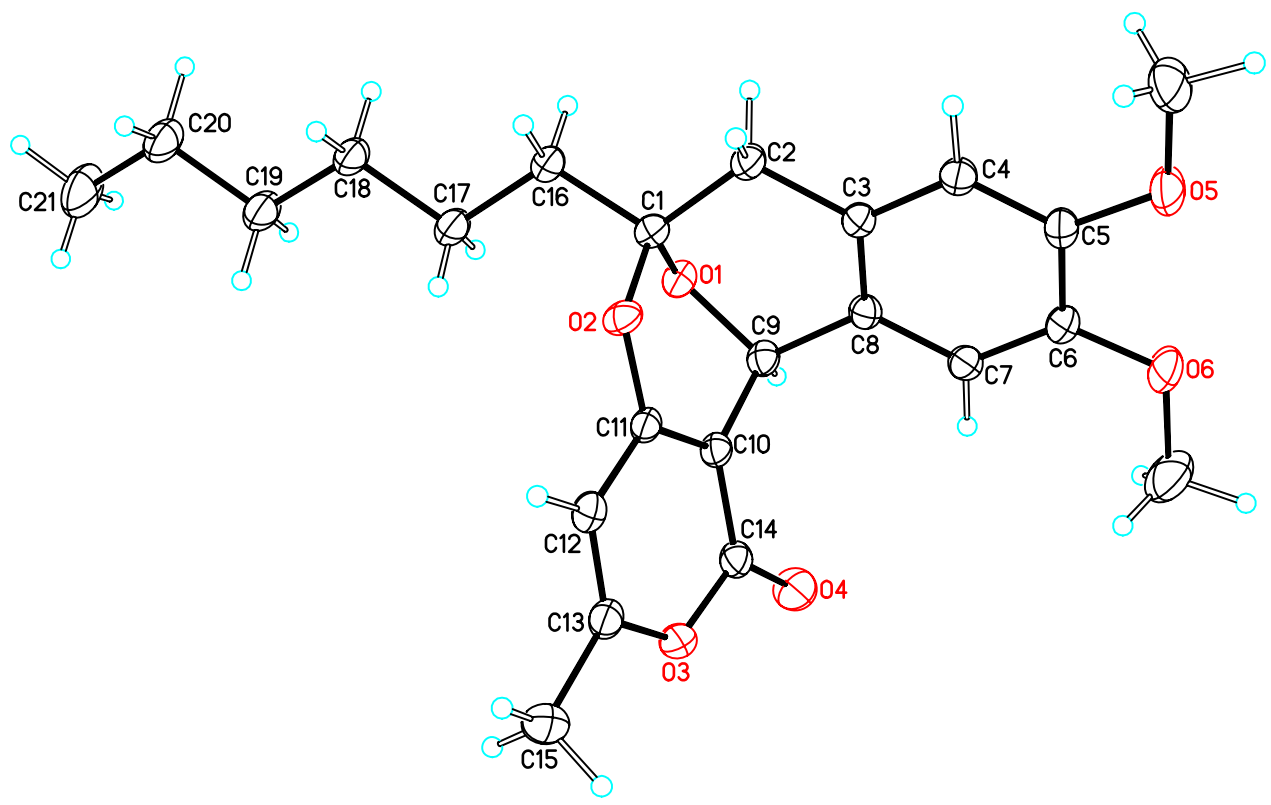

Table 1. Crystal data and structure refinement for 57.

Identification code

Empirical formula

Formula weight

Temperature

Wavelength

Crystal system

Space group

Unit cell dimensions

Volume

Z

Density (calculated)

Absorption coefficient
57

C23 H28 O6

400.45

173(2) K

$0.71073 \AA$

Triclinic

P-1

$$
\begin{array}{ll}
\mathrm{a}=7.1639(14) \AA & \alpha=106.268(10)^{\circ} . \\
\mathrm{b}=11.318(2) \AA & \beta=90.217(11)^{\circ} . \\
\mathrm{c}=14.060(3) \AA & \gamma=104.607(11)^{\circ} .
\end{array}
$$

2

$1.260 \mathrm{Mg} / \mathrm{m}^{3}$

$0.090 \mathrm{~mm}^{-1}$ 
$\mathrm{F}(000)$

Crystal size

Theta range for data collection

Index ranges

Reflections collected

Independent reflections

Completeness to theta $=30.52^{\circ}$

Absorption correction

Max. and min. transmission

Refinement method

Data / restraints / parameters

Goodness-of-fit on $\mathrm{F}^{2}$

Final $\mathrm{R}$ indices $[\mathrm{I}>2 \operatorname{sigma}(\mathrm{I})]$

$\mathrm{R}$ indices (all data)

Largest diff. peak and hole
428

$0.60 \times 0.20 \times 0.15 \mathrm{~mm}^{3}$

1.51 to $30.52^{\circ}$.

$-10<=\mathrm{h}<=10,-16<=\mathrm{k}<=16,-19<=\mathrm{l}<=19$

29410

$6313[\mathrm{R}(\mathrm{int})=0.0301]$

$98.0 \%$

Semi-empirical from equivalents

0.9866 and 0.9478

Full-matrix least-squares on $\mathrm{F}^{2}$

$6313 / 0 / 330$

1.033

$\mathrm{R} 1=0.0469, \mathrm{wR} 2=0.1390$

$\mathrm{R} 1=0.0575, \mathrm{wR} 2=0.1485$

0.885 and -0.351 e. $\AA^{-3}$

Table 2. Atomic coordinates $\left(\mathrm{x} 10^{4}\right)$ and equivalent isotropic displacement parameters $\left(\AA^{2} \times 10^{3}\right)$ for 57. $U(e q)$ is defined as one third of the trace of the orthogonalized $U^{i j}$ tensor.

\begin{tabular}{|c|c|c|c|c|}
\hline & $\mathrm{x}$ & $\mathrm{y}$ & $\mathrm{z}$ & $\mathrm{U}(\mathrm{eq})$ \\
\hline $\mathrm{O}(1)$ & $5167(1)$ & $4302(1)$ & $1150(1)$ & $21(1)$ \\
\hline $\mathrm{O}(2)$ & $2980(1)$ & $4847(1)$ & $2363(1)$ & $21(1)$ \\
\hline $\mathrm{O}(3)$ & $5606(1)$ & $3292(1)$ & $4132(1)$ & $25(1)$ \\
\hline $\mathrm{O}(4)$ & $8361(1)$ & $3650(1)$ & $3411(1)$ & $35(1)$ \\
\hline $\mathrm{O}(5)$ & $10063(1)$ & $10043(1)$ & $3217(1)$ & $39(1)$ \\
\hline $\mathrm{O}(6)$ & $12005(1)$ & $8570(1)$ & $3630(1)$ & $44(1)$ \\
\hline $\mathrm{C}(1)$ & $3734(1)$ & 4961(1) & $1398(1)$ & $20(1)$ \\
\hline $\mathrm{C}(2)$ & $4545(2)$ & $6371(1)$ & $1500(1)$ & $23(1)$ \\
\hline $\mathrm{C}(3)$ & $6538(1)$ & $6915(1)$ & $2063(1)$ & $20(1)$ \\
\hline C(4) & $7343(2)$ & $8240(1)$ & $2378(1)$ & $24(1)$ \\
\hline$C(5)$ & $9172(2)$ & $8767(1)$ & $2890(1)$ & $26(1)$ \\
\hline$C(6)$ & $10237(2)$ & $7960(1)$ & $3103(1)$ & $25(1)$ \\
\hline$C(7)$ & $9462(1)$ & $6648(1)$ & $2775(1)$ & $21(1)$ \\
\hline $\mathrm{C}(8)$ & $7608(1)$ & $6124(1)$ & $2252(1)$ & $18(1)$ \\
\hline $\mathrm{C}(9)$ & $6706(1)$ & $4695(1)$ & $1956(1)$ & $19(1)$ \\
\hline$C(10)$ & $5771(1)$ & $4276(1)$ & $2806(1)$ & $18(1)$ \\
\hline
\end{tabular}




$\begin{array}{lrrrr}\mathrm{C}(11) & 3926(1) & 4368(1) & 2942(1) & 18(1) \\ \mathrm{C}(12) & 2886(1) & 3951(1) & 3707(1) & 21(1) \\ \mathrm{C}(13) & 3754(2) & 3399(1) & 4256(1) & 23(1) \\ \mathrm{C}(14) & 6710(1) & 3753(1) & 3431(1) & 22(1) \\ \mathrm{C}(15) & 2841(2) & 2797(1) & 5019(1) & 34(1) \\ \mathrm{C}(16) & 1995(2) & 4335(1) & 621(1) & 24(1) \\ \mathrm{C}(17) & 1091(2) & 2919(1) & 482(1) & 29(1) \\ \mathrm{C}(18) & -743(2) & 2408(1) & -244(1) & 25(1) \\ \mathrm{C}(19) & -1591(2) & 969(1) & -498(1) & 31(1) \\ \mathrm{C}(20) & -3431(2) & 478(1) & -1218(1) & 33(1) \\ \mathrm{C}(21) & -4196(2) & -970(1) & -1535(1) & 47(1) \\ \mathrm{C}(22) & 9006(2) & 10869(1) & 3001(1) & 46(1) \\ \mathrm{C}(23) & 12884(2) & 7887(2) & 4120(1) & 43(1)\end{array}$

